\title{
On differential systems, graded Lie algebras and pseudo-groups
}

\author{
By \\ Noboru TANAKa \\ Dedicated to Prof. Atsuo Komatsu \\ for his 60th birthday
}

(Received Aug. 8, 1969)

\section{Introduction}

A differential system (or Pfaffian system) $D$ on a manifold $M$ is a law which assigns to every point $x \in M$ a subspace $D(x)$ of a given dimension of the tangent space $T_{x}(M)$ to $M$ at $x$ and which is differentiable in a suitable sense, or it may be simply defined as a subbundle of the tangent bundle $T(M)$ of $M$. Let $D$ (resp. $D^{\prime}$ ) be a differential system on a manifold $M\left(\right.$ resp. $M^{\prime}$ ). Then a diffeomorphism $\varphi$ of $M$ onto $M^{\prime}$ is called an isomorphism of $(M, D)$ onto $\left(M^{\prime}, D^{\prime}\right)$ if it induces a bundle isomorphism of $D$ onto $D^{\prime}$, i.e., $\varphi_{*} D(x)=D^{\prime}(\varphi(x))$ at each $x$ $\in M$.

The geometry of differential systems may be described as usual as a geometry of linear group structures ( $G$-structures) whose structure group $G$ is of infinite type and even not elliptic. This fact makes the geometry rather difficult to be studied on the basis of the usual theory of linear group structures. (By the usual theory, we here mean the local theory as appears in Singer and Sternberg [9] and the global theory (cf. Ruh [8] and Ochiai [7]) based on the theory of elliptic differential equations.) For instance, it seems to us that a direct use of the usual theory fails to give any finiteness theorem on the automorphism groups of differential systems. The same remark holds for other 
geometries based on the geometry of differential systems, e.g., for the geometry of pseudo-complex structures (=the geometry of real submanifolds of complex manifolds, see $\S 10)$.

In the previous paper [13], we have established a new prolongation scheme which can be well applied to a class of differential systems, to a class of pseudo-complex structures and generally to a class of linear group structures subordinate to differential systems. As an application, we have obtained a finiteness theorem for these structures.

The main purpose of the present paper is to give a further generalization of this prolongation scheme and to make some studies on graded Lie algebras, pseudo-groups, etc, which are closely related to the equivalence problem.

Let us now proceed to the description of the various sections and explain the main results in the present paper.

$\S 1$ is concerned which the assumptions to impose on the differential systems. We first introduce the notion of a regular differential system (Def. 1.1): A differential system $D$ on a manifold $M$ is called regular if there is a family $\left(D^{p}\right)_{p<0}$ of differential systems on $M$ such that $D^{-1}$ $=D$ and

$$
D^{p}=\left[D^{p+1}, D^{-1}\right]+D^{p+1}
$$

where $D^{r}$ is the sheaf of local cross-sections (local vector fields) of the vector bundle $D^{r}$. If $D$ is regular, then the family $\left(D^{p}\right)$ is uniquely determined and there is an integer $\mu>0$ such that $\cdots=D^{-\mu} \supsetneq \cdots \supsetneq D^{-1}$ $=D$. Throughout the present paper, we shall be mainly concerned with regular differential systems $D$ with $D^{-\mu}=T(M)$. We next introduce the notion of a fundamental graded (Lie) algebra (Def. 1.3): A graded Lie algebra $\mathfrak{m}=\sum_{p<0} \mathfrak{g}^{p}$, the indices $p$ taking values $<0$, is called fundamental if it satisfies the following conditions:

(1) $\operatorname{dim} n \mathrm{t}<\infty$;

(2) $\mathrm{m}$ is generated by $\mathfrak{g}^{-1}$, i.e., $\left[\mathfrak{g}^{p}, \mathfrak{g}^{-1}\right]=\mathfrak{g}^{p-1}$.

Moreover a fundamental graded algebra is called of $\mu$-th kind if $\mathfrak{g}^{p}$ 
$\{0\}$ for all $p<-\mu$ and $\mathfrak{g}^{-\mu} \neq\{0\}$. Let $D$ be a regular differential system on $M$. Then it is shown that the direct $\operatorname{sum} m(x)=\sum_{p<0} D^{p}(x) /$ $D^{p+1}(x)$ is endowed with a structure of fundamental graded algebra (of $\mu$-th kind). Given a fundamental graded algebra $\mu$, we say that $D$ is of type $\Pi$ if every $\mathrm{ml}(x)$ is isomorphic with $\mathrm{m}$ as graded algebras (Def. 1.4). For example, every homogeneous differential system $D$ is regular and of type $m$ with some $m$.

In $\S 2$, we define the standard differential systems. Let $m=\sum_{p<0} g^{p}$ be a fundamental graded algebra and let $M(\mathrm{ml})$ be the simply connected Lie group whose Lie algebra is given by $n$. Then the subspace $\mathrm{g}^{-1}$ of $\mathrm{m}$ defines a left-invariant differential system $D$ on $M(\mathrm{~m})$, which is regular and of type $\mathrm{m}$. This differential system $D$ is called the standard differential system of type $\mathrm{nt}$. For example, every contact structure is locally equivalent to a standard differential system. In $\S 3$, we argue about the "universal" fundamental graded algebras, which leads to the study of "generic" differential systems.

In $\S 4$, we generalize the notion of regular differential systems of

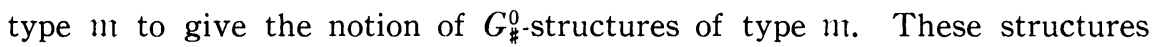
are the very linear group structures on which we discuss the equivalence problem. We here only explain how to define the group $G_{\sharp}^{0}$. Let $\mathrm{m}$ $=\sum_{p<0} \mathfrak{g}^{p}$ be a fundamental graded algebra and let $G^{0}(n)$ be the automorphism group of $m$ as graded algebra. Let $N^{0}$ be the subgroup of $G L(\mathrm{~m})$ consisting of all $a \in G L(n 1)$ such that $a X \equiv X\left(\bmod \mathfrak{D}^{p+1}\right)$ for all $X \in \mathfrak{D}^{p}$ and $p<0$, where $\mathfrak{D}^{p}=\sum_{r=p}^{-1} \mathrm{~g}^{r}$. Given a Lie subgroup $G^{0}$ of $G^{0}(m)$, then the group $G_{\sharp}^{0}$ is defined to be the product $G^{0} \cdot N^{0}$ of $G^{0}$ and $N^{0}$, being a Lie subgroup of $G L(\mathrm{~m})$. Note that a regular differential system of type $\mathrm{n}$ may be described as a $G_{\sharp}^{0}(\mathrm{n})$-structure of type $\|$, where $G_{\sharp}^{0}(\mathrm{~m})$ $=G^{0}(\mathrm{~m}) \cdot N^{0}$.

$\S 5$ is concerned with the algebraic prolongations of fundamental graded algebras, etc. It is shown that every fundamental graded algebra $\mathfrak{m}=\sum_{p<0} \mathfrak{g}^{p}$ is prolonged to a graded algebra $\mathfrak{g}(\mathfrak{m})=\sum_{p<0} \mathfrak{g}^{p}+\sum_{p \geqq 0} \mathfrak{g}^{p}(\mathfrak{m})$. Note that $\mathfrak{g}^{0}(\mathfrak{m})$ is the Lie algebra of all derivations of $\mathfrak{m}$ as graded algebra, i.e., the Lie algebra of $G^{0}(\mathrm{~m})$. In general, let $\mathrm{g}^{0}$ be a subalgebra of 
$g^{0}(\mathfrak{m})$. Then the direct sum $\sum_{p \leqq 0} \mathrm{~g}^{p}$ is prolonged to a graded subalgebra $\mathfrak{g}=\sum_{p} \mathfrak{g}^{p}$ of $\mathfrak{g}(\mathfrak{m})$, which is called the prolongation of $\left(\mathfrak{n}, \mathfrak{g}^{0}\right)$. We say that $\left(\mathfrak{n}, \mathfrak{g}^{0}\right)$ is of infinite type (resp. of finite type) if $\mathfrak{g}^{p} \neq\{0\}$ for all $p \geqq 0$ (resp. if $\mathrm{g}^{p}=\{0\}$ for some $p \geqq 0$ ).

In $\S 6$, we study the infinitesimal automorphisms of the standard differential system $D$ of type "I. We prove that the formal algebra of all formal infinitesimal automorphisms of $(M(\mathrm{mI}), D)$ is the completion $\overline{\mathfrak{g}(\mathfrak{m})}$ of $\mathfrak{g}(\mathfrak{m})$ (Th. 6.1).

$\S 7$ is preliminary to the subsequent sections. Let $m=\sum_{p<0} \mathrm{~g}^{p}$ be a fundamental graded algebra and let $G^{0}$ be a Lie subgroup of $G^{0}(\mathrm{~m})$. Let $\mathfrak{g}^{0}$ be the Lie algebra of $G^{0}$ which is a subalgebra of $g^{0}(n 1)$ and let $\mathfrak{g}$ $=\sum_{p} \mathfrak{g}^{p}$ be the prolongation of $\left(n, \mathfrak{g}^{0}\right)$. We first define the groups $G^{k}$ $=G_{\sharp}^{k} / N^{k}(k>0)$ by using the prolongation of $\left(\mathrm{m}, \mathrm{g}^{0}\right)$ and then introduce the notion of a pseudo- $G^{k}$-structure of type $m$ (Def. 7.2). A pseudo- $G^{k}$. structure of type $m$ on a manifold $M$ is a triple $\left(P^{k}, D^{(k)}, \theta^{(k)}\right)$ as follows: $P^{k}$ is a principal fiber bundle over the base space $M$ with structure group $G^{k} ; D^{(k)}$ is a family of differential systems on $P^{k} ; \theta^{(k)}$ is a family of "pseudo-1-forms" on $P^{k}$, the "fundamental form" of this structure. It should be remarked that a $G_{\sharp}^{0}$-structure of type $\mathrm{m}, P_{\sharp}^{0}$, on a manifold $M$ may be described as a pseudo- $G^{0}$-structure of type $n$, $\left(P^{0}, D^{(0)}, \theta^{(0)}\right)$, on $M($ cf. Th. 8.1).

$\S 8$ is mainly devoted to the description of the prolongation theorem for pseudo- $G^{0}$-structures of type $\ldots$. The prolongation theorem is roughly stated as follows (Th. 8.3):

(1) With every pseudo- $G^{0}$-structure of type $n$, $\left(P^{0}, D^{(0)}, \theta^{(0)}\right)$, on a manifold $M$, there is associated a sequence

$(P): \quad\left(P^{0}, D^{(0)}, \theta^{(0)}\right) \leftarrow \cdots \leftarrow\left(P^{k}, D^{(k)}, \theta^{(k)}\right) \leftarrow \cdots$

of pseudo- $G^{k}$-structures of type $\mathrm{nt}$, where $\left(P^{k}, D^{(k)}, \theta^{(k)}\right)$ is a pseudo$G^{k}$-structure of type $\mathrm{m}$ on $P^{k-1}$;

(2) The assignment $\left(P^{0}, D^{(0)}, \theta^{(0)}\right) \rightarrow(P)$ is compatible with the various isomorphisms.

As an immediate corollary, we have the following finiteness theorem 


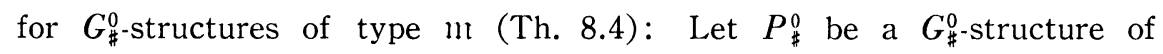
type $\mathrm{m}$ on a connected manifold $M$. If $\left(\mathrm{m}, \mathrm{g}^{0}\right)$ is of finite type, then the Lie algebra of all infinitesimal automorphisms of $P_{\#}^{0}$ is of finite dimension $\leqq \operatorname{dim} \mathfrak{g}$. In this connection, we remark that the group $G_{\#}^{0}$ is of infinite type and not elliptic unless $m$ is of first kind. Th. 8.4 generalizes the finiteness theorems contained in [1], [9], [11] and [13]. In $\S 9$, we construct sequence $(P)$ and prove the prolongation theorem.

In $\S 10$, we first introduce the notion of a pseudo-complex structure. Let $D$ be a differential system on a manifold $M$ and let $I$ be a crosssection of the vector bundle $\operatorname{Hom}(D, D)$. Then the pair $(D, I)$ is called a pseudo-complex structure on $M$ if $I_{x}^{2}(Y)=-Y$ for all $Y$ $\in D(x)$ and if, for any two local cross-sections $X, Y$ of $D$,

(1) $[I X, I Y]-[X, Y]$ is a local cross-section of $D$;

(2) $[I X, I Y]-[X, Y]=I([I X, Y]+[X, I Y])$.

We then clearify the close relation between the geometry of pseudocomplex structures and that of real submanifolds of complex manifolds and apply our main theorems to the former geometry. Recently Tanaka [14] has applied the results in $\S 10$ combined with those in $\S \S 5$ and 6 to the determination of infinitesimal automorphisms of Siegel domains.

Finally in $\S 11$, we study certain graded modules (over non-commutative rings) and apply the result to some problems on certain graded Lie algebras. Let $\mathfrak{m}=\sum_{p<0} \mathfrak{g}^{p}$ be a fundamental graded algebra over a field $K$ of characteristic zero and let $E$ be a (right) m-module. Assume that $E$ is graded: $E=\sum_{p} E^{p}($ direct sum $) ; E^{p} \mathfrak{g}^{r} \subset E^{p+r} ; \operatorname{dim} E^{p}<\infty$. Moreover assume that

(1) $E^{p}=\{0\}$ for all $p<-\mu$ if $n$ is of $\mu$-th kind;

(2) for each $p \geqq 0$, the condition " $a \in E^{p}, a \mathfrak{g}^{-1}=\{0\}$ ” implies $a=0$. For each $p \geqq 0$, let $H^{p}(E)$ be the subspace of $E^{p}$ consisting of all $a$ $\in E^{p}$ such that $a \mathrm{~g}^{r}=\{0\}$ for all $r<-1$. Then we prove that $E^{p} \neq\{0\}$ for all $p \geqq 0$ if and only if $H^{p}(E) \neq\{0\}$ for all $p \geqq 0$ (Th. 11.1). Th. 11.1 will be of some interest relating to the algebraic theory of partial 
differential equations. As an immediate corollary, we obtain: Let $m$ $\underset{p<0}{=} \sum \mathfrak{g}^{p}$ be a fundamental graded algebra and let $\mathrm{g}^{0}$ be a subalgebra of $\mathfrak{g}^{0}(\mathfrak{m})$. Let $\mathfrak{h}^{0}$ be the ideal of $\mathfrak{g}^{0}$ consisting of all $X \in \mathfrak{g}^{0}$ such that $\left[X, g^{r}\right]=\{0\}$ for all $r<-1$ and identify $\mathfrak{h}^{0}$ with a subspace of Hom $\left(\mathfrak{a}^{-1}, \mathfrak{g}^{-1}\right)$ ((by identifying $X \in \mathfrak{h}^{0}$ with the endomorphism $\mathfrak{g}^{-1} \ni Y \rightarrow$ $\left.[X, Y] \in \mathfrak{g}^{-1}\right)$. Then $\left(\mathfrak{m}, \mathfrak{g}^{0}\right)$ is of infinite type if and only if the subspace $\mathfrak{h}^{0}\left(\operatorname{Hom}\left(\mathfrak{g}^{-1}, \mathfrak{g}^{-1}\right)\right.$ is of infinite type (Cor. 2 to Th. 11.1). This result is of much importance in connection with the finiteness theorem stated above and plays a fundamental role in the classification of primitive infinite pseudo-groups (see Morimoto and Tanaka [6]).

\section{Contents}

$\S 1$. Strongly regular differential systems

$\S 2$. The standard differential system of type $m$

$\S 3$. The universal fundamental graded algebras

$\S 4 . \quad G_{\sharp}^{0}$-structures of type $\mathrm{m}$

$\S 5$. The algebraic prolongations

§ 6. Infinitesimal automorphisms of the standard differeatial system of type $\mathrm{nt}$

$\S 7$. $\mathrm{G}_{\sharp}^{k}$-structures of type $\mathfrak{n t}$ and pseudo- $G^{k}$-structures of type $\mathfrak{n t}$

$\S 8$. The prolongation theorems

§ 9. Proof of Theorem 8.1, Theorem 8.2 and of Lemma 8.2

\$10. Applications to the geometry of real submanifolds of complex manifolds

$\$ 11$. Some results on certain graded modules

\section{Preliminary remarks}

1. Definition 1. Let $\mathfrak{g}$ be a Lie algebra over a field $K$. Assume that there is given a family $\left(\mathfrak{g}^{p}\right)_{p \in \boldsymbol{Z}}$ of subspaces of $\mathfrak{g}$ which satisfies the following conditions, where $\boldsymbol{Z}$ denotes the additive group of integers:

1) $\mathfrak{g}=\sum_{p} \mathfrak{g}^{p}$ (direct sum);

2) $\operatorname{dim} \mathfrak{g}^{p}<\infty$;

3) $\left[\mathfrak{g}^{p}, \mathfrak{g}^{q}\right] \subset \mathfrak{g}^{p+q}$.

Under these conditions, we say that the system $\left\{\mathfrak{g},\left(\mathfrak{g}^{p}\right)\right\}$ or the direct sum $\mathfrak{g}=\sum_{p} \mathfrak{g}^{p}$ or simply $\mathfrak{g}$ is a graded algebra. 
Moreover we define the notion of homomorphism and of isomorphism for graded algebras in an obvious manner.

Let $\mathfrak{g}$ be a Lie algebra and assume that there is given a family $\left(\mathfrak{g}^{p}\right)_{k \leqq p \leqq l}$ of subspaces of $\mathfrak{g}$ such that $\mathfrak{g}=\sum_{p=k}^{l} \mathfrak{g}^{p}$ (direct sum). Then we shall say that the direct sum $\mathfrak{g}=\sum_{p=k}^{l} \mathfrak{g}^{p}$ is a graded algebra if $\mathfrak{g}=\sum_{p \in \mathbb{Z}} \mathfrak{g}^{p}$ becomes a graded algebra by putting $\mathrm{g}^{p}=\{0\}$ for any $p\left(p<k\right.$ or $\left.p^{p \in Z}>l\right)$. Let $\mathfrak{g}=\sum_{p} \mathfrak{g}^{p}$ be a graded algebra. A subalgebra $\mathfrak{h}$ of the Lie algebra 9 will be called a graded subalgebra if we have $\mathfrak{h}=\sum_{p} \mathfrak{h} \cap \mathfrak{g}^{p}$.

2. Throughout this paper, we shall always assume the differentiability of class $C^{\infty}$ unless otherwise stated. Given a manifold $M, T_{x}(M)$ will denote the tangent space to $M$ at a point $x \in M$.

Definition 2. (1) An $n$-dimensional differential system $D$ on a manifold $M$ is a differentiable mapping $D$ which assigns to every point $x \in M$ an $n$-dimensional subspace $D(x)$ of $T_{x}(M)$.

(2) Let $D$ (resp. $D^{\prime}$ ) be a differential system on a manifold $M$ (resp. $M^{\prime}$ ). A diffeomorphism $\varphi$ of $M$ onto $M^{\prime}$ is called an isomorphism of $(M, D)$ onto $\left(M^{\prime}, D^{\prime}\right)$ if we have $\varphi_{*} D(x)=D^{\prime}(\varphi(x))$ at each $x \in M$, $\varphi_{*}$ being the differential of $\varphi$.

3. As for differential forms defined on differential systems, principal fiber bundles and $G$-structures, we shall adopt the definitions and notations given in $[13], \S 1$.

\section{§1. Strongly regular differential systems}

1.1. Let $M$ be a manifold. We denote by $T(M)$ the tangent bundle of $M$. Every differential system $D$ on $M$ may be identified with a vector subbundle of $T(M)$, and vice versa. Given a differential system $D$ on $M$, we denote by $\underline{D}$ the sheaf of all local cross-sections of the vector bundle $D$. The sheaf $\underline{T(M)}$ is nothing but the Lie algebra sheaf of all local vector fields on $M$, and the sheaf $\underline{D}$ is a vector subsheaf of $\underline{T(M)}$. In general, let $\mathscr{D}$ be a vector subsheaf of $\underline{T(M)} . \mathscr{D}(x)$ denotes the stalk of $\mathscr{D}$ at a point $x \in M$. Given a local cross-section $X$ of $\mathscr{D}$ defined on a neighborhood of $x, \underline{X}_{x}$ denotes the germ at $x$ 
represented by $X$, which is an element of $\mathscr{D}(x)$.

Let $D$ and $D^{\prime}$ be two differential systems on $M$ such that $D \subset D^{\prime}$, i.e., $D(x) \subset D^{\prime}(x)$ at each $x \in M$. Let $x$ be any point of $M$. We denote by $\mathscr{E}(x)$ the vector subspace of $T(M)(x)$ spanned by all the elements of the form $\left[\underline{X}_{x}, \underline{Y}_{x}\right]+\underline{Z}_{x}$ where $\underline{X}_{x}, \underline{Z}_{x} \in \underline{D}^{\prime}(x)$ and $\underline{Y}_{x} \in \underline{D}(x)$, i.e., $\mathscr{E}(x)=\left[\underline{D}^{\prime}(x), \underline{D}(x)\right]+\underline{D}^{\prime}(x)$. Moreover we denote by $D^{\prime \prime}(x)$ the subspace of $T_{x}(M)$ consisting of all the elements $X_{x}$, where $\underline{X}_{x} \in \mathscr{E}(x)$. Then we say that the pair $\left(D^{\prime}, D\right)$ is regular if $\operatorname{dim} D^{\prime \prime}(x)$ is constant. If $\left(D^{\prime}, D\right)$ is regular, then we see that the assignment $M \ni x \rightarrow D^{\prime \prime}(x)$ gives a differential system $D^{\prime \prime}$ on $M$ and that the assignment $M \ni x \rightarrow$ $\mathscr{E}(x)$ gives the sheaf $D^{\prime \prime}$ associated with $D^{\prime \prime}$. We have $D^{\prime \prime} \supset D^{\prime} \supset D$. For the moment, the differential system $D^{\prime \prime}$ will be denoted by $D^{\prime} \square D$. The differential system $D \square D$ is called the derived system of $D$ (cf. $[2]) . \quad D$ is completely integrable if and only if $[\underline{D}(x), \underline{D}(x)] \subset \underline{D}(x)$ at each $x \in M$ or equivalently $D \square D=D$.

Definition 1.1. We say that a differential system $D$ on $M$ is regular if there is a family $\left(D^{p}\right)_{p<0}$ of differential systems on $M$ satisfying the following conditions:

1) $\cdots \supset D^{p-1} \supset D^{p} \supset \cdots \supset D^{-1}=D$;

2) For each $p<0$, the pair $\left(D^{p}, D^{-1}\right)$ is regular and $D^{p-1}$ $=D^{p} \square D^{-1}$.

It is clear that the family $\left(D^{p}\right)_{p<0}$ satisfying the above conditions 1) and 2) is uniquely determined by $D$. Since $\operatorname{dim} D^{p} \leqq \operatorname{dim} M$, there is an integer $\mu>0$ such that

$$
\cdots=D^{-\mu-1}=D^{-\mu} \supsetneq D^{\mu+1} \supsetneq \cdots \supsetneq D^{-1} .
$$

Definition 1.2. $\mu$ being just as above, the regular differential system $D$ is called of $\mu$-th kind.

We say that a differential system $D$ on $M$ is homogeneous if the pseudo-group of all the local automorphisms of $(M, D)$ is transitive on $M$. It is obvious that a homogeneous differential system $D$ is regular. 
Remark. Our regularity condition for differential systems is a general one. In fact, it is easy to see that any differential system $D$ on $M$ is regular on some open set $U$ of $M$.

Proposition 1.1. Let $D$ be a regular differential system of $\mu-t h$ kind on $M$. Then the differential system $D^{-\mu}$ is the smallest completely integrable differential system on $M$ containing $D$.

Proof. We have $\left[\underline{D}^{p}(x), \underline{D}^{-1}(x)\right] \subset \underline{D}^{p-1}(x)$ at each $x \in M$. It follows that $\left[\underline{D}^{r}(x), \underline{D}^{s}(x)\right] \subset \underline{D}^{r+s}(x)$. Prop. 1.1 is easy from this fact.

Let $D$ be a regular differential system of $\mu$-th kind on $M$, and let $N$ be a maximal integral manifold of $D^{-\mu}$. Then each differential system $D^{p}$ can be restricted to $N$, which we denote by $D^{p} \mid N$. Then it is clear that $D\left|N=D^{-1}\right| N$ is regular and that $\left(D^{p} \mid N\right)_{p<0}$ is just the family of differential systems associated with $D \mid N$. Moreover, we see that $D \mid N$ is of $\mu$-th kind and $D^{-\mu} \mid N=T(N)$. In this paper, we shall be mainly concerned with regular differential systems $D$ of $\mu$-th kind with $D^{-\mu}=T(M)$.

1.2. Let $D$ be a regular differential system on $M$ and let $x$ be any point of $M$. We set $\mathfrak{g}^{p}(x)=D^{p}(x) / D^{p+1}(x)$ and $\mathfrak{m}(x)=\sum_{p<0} \mathfrak{g}^{p}(x)$ (direct sum). Let us define, in a natural manner, a bracket operation in $\mathfrak{m}(x)$ so that $\mathfrak{m}(x)$ becomes a graded algebra. For this purpose, we set $\underline{\mathfrak{g}}^{p}(x)=\underline{D}^{p}(x) / \underline{D}^{p+1}(x)$ and $\underline{m}^{\prime}(x)=\sum_{p<0} \mathfrak{g}^{p}(x)$ (direct sum). Since $\left[\underline{D}^{r}(x), \underline{D}^{s}(x)\right] \subset \underline{D}^{r+s}(x)$, the bracket operation $[$,$] in \underline{T(M)}(x)$ induces a bracket operation $[$,$] in \underline{m}(x)$ in such a way that $m(x)$ becomes a "graded algebra". (Note that $\left.\operatorname{dim} \underline{\mathfrak{g}}^{p}(x)=\infty\right)$. Since $\left[\underline{D}^{p}(x)\right.$, $\left.\underline{D}^{-1}(x)\right]+\underline{D}^{p}(x)=\underline{D}^{p-1}(x)$, we have $\left[\underline{\mathfrak{q}}^{p}(x), \underline{\mathfrak{q}}^{-1}(x)\right]=\mathfrak{q}^{p-1}(x)$. Let $\alpha^{p}$ (resp. $\underline{\alpha}^{p}$ ) denote that projection of $D^{p}(x)$ (resp. $\underline{D}^{p}(x)$ ) onto $\mathfrak{g}^{p}(x)$ (resp. $\underline{\mathrm{q}}^{p}(x)$ ). The mapping $\underline{D}^{p}(x) \ni \underline{X}_{x} \rightarrow X_{x} \in D^{p}(x)$ induces a linear mapping $\beta^{p}$ of $\underline{g}^{p}(x)$ onto $\mathrm{g}^{p}(x)$ such that $\beta^{p}\left(\underline{\alpha}^{p}\left(\underline{X}_{x}\right)\right)=\alpha^{p}\left(X_{x}\right)$ for any $\underline{X}_{x} \in \underline{D}^{p}(x)$. The family $\left(\beta^{p}\right)_{p<0}$ defines a linear mapping $\beta$ of $\underline{\mathfrak{m}}(x)$ onto $\mathrm{m}(x)$.

Lemma 1.1. Let $\underline{X}_{x} \in \underline{D}^{p}(x)$ and $\underline{Y}_{x} \in \underline{D}^{q}(x)$. 
(1) If $X_{x}=0$, then we have $[X, Y]_{x} \in D^{p+q+1}(x)$.

(2) If $X_{x} \in D^{p+1}(x)$, then we have $[X, Y]_{x} \in D^{p+q+1}(x)$.

Proof. (1) Let $\omega^{1}=\cdots=\omega^{k}=0$ be a local equation of $D^{p+q+1}$ at $x$. We have $\omega^{i}\left([X, Y]_{x}\right)=-d \omega^{i}\left(X_{x} \wedge Y_{x}\right)+X_{x} \omega^{i}(Y)-Y_{x} \omega^{i}(X)$. Since $X_{x}=0$ and $D^{p} C D^{p+q+1}$, we get $\omega^{i}\left([X, Y]_{x}=0(1 \leqq i \leqq k)\right.$, which means $[X, Y]_{x} \in D^{p+q+1}(x)$. (2) follows easily from (1) and the fact that $\left[\underline{D}^{p+1}(x), \underline{D}^{q}(x)\right] \subset D^{p \div q * 1}(x)$.

By Lemma 1.1, we know that there is a unique bracket operation $[$,$] in m(x)$ such that $m(x)$ becomes a graded algebra and such that $\beta$ gives a homomorphism of $\underline{11}(x)$ onto $\Pi 1(x)$ as graded algebras. Since $\left[\underline{\mathfrak{g}}^{p}(x), \underline{\mathfrak{g}}^{-1}(x)\right]=\underline{\mathfrak{g}}^{p-1}(x)$, we have $\left[\mathfrak{g}^{p}(x), \mathfrak{g}^{-1}(x)\right]=\mathfrak{g}^{p-1}(x)$.

Definition 1.3. (1) We say that a graded algebra $m=\sum_{p<0} \mathfrak{g}^{p}$ over a field $K$ is fundamental if it satisfies the following two conditions:

1) $\operatorname{dim} m<\infty$;

2) $\mathrm{m}$ is generated by $\mathfrak{g}^{-1}$, or more precisely $\left[\mathfrak{g}^{p}, \mathfrak{g}^{-1}\right]=\mathfrak{g}^{p-1}(p<0)$.

(2) We say that a fundamental graded algebra $\Pi=\sum_{p<0} \mathrm{~g}^{p}$ is of $\mu$-th kind if $\mathfrak{g}^{p}=\{0\}(p<-\mu)$ and $\mathfrak{g}^{-\mu} \neq\{0\}$.

By this definition, we see that the graded algebra $m(x)$, constructed as above, is fundamental.

Definition 1.4. Let $D$ be a regular differential system on $M$.

(1) $D$ is called strongly regular if the graded algebras $m(x)$ $(x \in M)$ are mutually isomorphic;

(2) Let $\sharp=\sum_{p<0} \mathfrak{g}^{p}$ be a fundamental graded algebra over the field $\boldsymbol{R}$ of real numbers. Then, $D$ is called of type $m$ if the graded algebra $\| I(x)$ is isomorphic with the graded algebra $\|$ at each $x \in M$.

It is clear that homogeneous differential systems are strongly regular. In $§ 3$, we shall see that "generic" differential systems of some kind are also strongly regular.

Examples. (1) Let $m=\mathfrak{g}^{-2}+\mathfrak{g}^{-1}$ be a fundamental graded algebra of second kind, where $\operatorname{dim} \mathfrak{g}^{-2}=1$ and $\operatorname{dim} \mathfrak{g}^{-1}=n$. Let $e_{0}$ be a base of 
$\mathfrak{g}^{-2}$ and define an anti-symmetric bilinear form $B$ on $\mathfrak{g}^{-1}$ by $B\left(X^{-1}\right.$, $\left.Y^{-1}\right) e_{0}=\left[X^{-1}, Y^{-1}\right]$ for any $X^{-1}, Y^{-1} \in \mathfrak{g}^{-1}$. Let $r$ be an integer with $0<r \leqq\left[\frac{n}{2}\right]$. We say that $\|$ is of class $r$ (resp. non-degenerate) if rank $B=2 r$ (resp. if $B$ is non-degenerate). It is clear that there is a unique fundamental graded algebra $"$ It of class $r$ up to isomorphism. Let $M$ be a manifold of dimension $n+1$, and let $D$ be a differential system of dimension $n$ on $M$. Let $\|$ be a fundamental graded algebra of second kind, where $\operatorname{dim} \mathfrak{g}^{-2}=1$ and $\operatorname{dim} \mathfrak{g}^{-1}=n$. Then we say that $D$ is of class $r$ (resp. non-degenerate), if $n$ is of class $r$ (resp. nondegenerate) and if $D$ is of type $\Pi 1$. $D$ is also called a contact structure if it is non-degenerate.

(2) (cf. $[1]$, p. 936-938) Let $\Pi=\sum_{p=-\mu}^{-1} \mathrm{~g}^{p}$ be a fundamental graded algebra of $\mu$-th kind, where $\operatorname{dim} \mathfrak{g}^{-1}=2$ and $\operatorname{dim} \| \leqq 5$. Then we have the following five cases:

1) $\operatorname{dim} n=2, \mu=1\left(n^{-1}=2\right)$,

2) $\operatorname{dim} u=3, \mu=2\left(n^{-2}=1, n^{-1}=2\right)$,

3) $\operatorname{dim} n=4, \mu=3\left(n^{-3}=n^{-2}=1, n^{-1}=2\right)$,

4) $\operatorname{dim} u=5, \mu=3\left(n^{-3}=2, n^{-2}=1, n^{-1}=2\right)$,

5) $\operatorname{dim} n=5, \mu=4\left(n^{-4}=n^{-3}=n^{-2}=1, n^{-1}=2\right)$,

where we put $n^{p}=\operatorname{dim} g^{p}$. It can be easily shown that, in each case, there exists a unique fundamental graded algebra $" \prime t$ up to isomorphism. Let $M$ be a manifold of dimension 5 , and let $D$ be a regular differential system on $M$, where $\operatorname{dim} D=2$. Then we have $\operatorname{dim} \mathfrak{g}^{-1}(x)=2$ and $\operatorname{dim} m(x) \leqq 5$ at each $x \in M$. Therefore we know from the above argument that $D$ is strongly regular.

\section{§2. The standard differential system of type $m$}

2.1. Let $m=\sum_{p<0} \mathfrak{g}^{p}$ be a fundamental graded algebra of $\mu$-th kind over $\boldsymbol{R}$. Let $M(\mathrm{~m})$ be the simply connected Lie group whose Lie algebra is given by $\mathrm{m}$. (Hence $\mathrm{m}$ may be identified with the Lie algebra 
of all left invariant vector fields on the Lie group $M(\mathfrak{m})$.) We set $\mathfrak{D}^{p}$ $=\sum_{r=p}^{-1} \mathfrak{g}^{r}$ and define a differential system $D^{p}$ on $M(n i)$ by $D^{p}(x)=\left(\grave{D}^{p}\right)_{x}$, the subspace of $T_{x}(M(\mathrm{n} \mathrm{l}))$ consisting of all the vectors $X_{x}\left(X \in \mathfrak{D}^{p}\right)$. Since $\left[\mathfrak{g}^{p}, \mathfrak{g}^{-1}\right]=\mathfrak{g}^{p-1}$, we easily have $\left[\underline{D}^{p}(x), \underline{D}^{-1}(x)\right]+\underline{D}^{p}(x)=\underline{D}^{p-1}(x)$. This shows that $D=D^{-1}$ is a regular differential system on $M(111)$ and that $\left(D^{p}\right)_{p<0}$ is the family of differential systems associated with $D$. Moreover it is clear that $D$ is strongly regular of type $n$. The differential system $D$ on $M(\mathrm{nt})$, thus obtained, is called the standard differential system of type nt. Let $\xi$ be the Maurer-Cartan form of the Lie group $M(\mathrm{~m})$ which is the $m$-valued 1 -form on $M(m)$ defined by $\xi\left(X_{x}\right)=X$ for any $X \in m$ and $x \in M(n)$. Let $\tilde{\xi}^{p}$ denote the $\mathrm{g}^{p}$-component of $\xi$ in the decomposition $m=\sum_{p<0} \mathrm{~g}^{p}$. Then it is clear that $D^{p}$ is defined by the equations $\xi^{r}=0(r<p)$.

2.2. Let us now realize the Lie group $M(n)$ as a Lie subgroup of the affine transformation group $A F(n)$ of $m$. Let $u^{p}$ denote the projection of $m$ onto $\mathfrak{g}^{p}$ in the decomposition $\| 1=\sum_{p<0} \mathfrak{g}^{p}$, which may be considered as a $\mathrm{g}^{p}$-valued function on $\mathrm{nt}$. Then the system $\left(u^{p}\right)_{-\mu \leqq p<0}$ defines a linear "coordinate system" of the manifold nt. (Note that $u^{p}$ $=0$ if $p<-\mu$.) For each $X \in m$, we define an infinitesimal affine transformation $s(X)$ of $\mathrm{m}$ by

$$
s(X)(Y)=X+\sum_{p, q<0} \frac{q}{p+q}\left[u^{p}(X), u^{q}(Y)\right]
$$

for all $Y \in m$. Then it can be easily shown that the mapping $X \rightarrow s(X)$ gives an injective homomorphism $s$ of the Lie algebra $m$ into the Lie algebra $a f(n t)$ of all infinitesimal affine transformations of 11 . Furthermore we see that $s$ generates an injective homomorphism $S$ of the Lie group $M(\mathrm{~m})$ into the Lie group $A F(\mathrm{~m})$.

2.3. The affine transformation group $A F(\mathrm{~m})$ may be expressed as the product $\mathrm{m} \times G L(\mathrm{~m})$; We denote by $\rho$ the projection of $A F(\mathrm{~m})$ onto mi. Then the mapping $f=\rho \circ S$ gives a diffeomorphism of $M$ ( $m$ ) onto i11. We have $f(a x)=S(a) f(x)$ for all $a, x \in M(i 11)$. It follows that 
the group $S(M(\mathrm{~m}))$ is simply transitive on $\mathrm{mt}$ and that the differential system $\hat{D}=\left(f^{-1}\right)^{*} D$ on $\mathrm{m}$ is invariant by $S(M(n))$. Moreover if we put $\hat{\xi}^{p}=\left(f^{-1}\right)^{*} \xi^{p}$, then $\hat{D}$ is defined by the equations $\hat{\xi}^{p}=0(p \leqq-2)$. For the sake of simplicity, assume that $\mu \leqq 3$, i.e., $m=\mathfrak{g}^{-3}+\mathfrak{g}^{-2}+\mathfrak{g}^{-1}$. If we put

$$
\begin{aligned}
\eta^{-1} & =d u^{-1} \\
\eta^{-2} & =d u^{-2}-\frac{1}{2}\left[u^{-1}, d u^{-1}\right], \\
\eta^{-3} & =d u^{-3}-\frac{1}{3}\left[u^{-2}, d u^{-1}\right]-\frac{2}{3}\left[u^{-1}, d u^{-2}\right] \\
& +\frac{1}{6}\left[u^{-1},\left[u^{-1}, d u^{-1}\right]\right],
\end{aligned}
$$

then it can be shown that $\hat{\xi}^{p}=\eta^{p}(p=-3,-2,-1)$. Thus we know that the differential system $\hat{D}$ is defined by the equations $\eta^{-3}=\eta^{-2}=0$, which may be adopted as the second definition of the standard differential system of type $n$ for the case $\mu \leqq 3$.

Examples. (1) The case where $\mu=2, \operatorname{dim} g^{-2}=1$ and where $m$ is of class $r$ (cf. 1.2, Example (1)). Let $e_{0}$ be a base of $g^{-2}$. Then we can find a base $e_{1}, \ldots, e_{n}$ of $\mathfrak{g}^{-1}$ such that $\left[e_{i}, e_{j}\right]=e_{0}(1 \leqq i \leqq r$, $j=r+i),=-e_{0}\left(i=r+j, 1 \leqq j \leqq r\right.$ ) and $=0$ (otherwise). Let $x^{0}, \ldots, x^{n}$ denote the coordinate system of $m$ corresponding to the base $e_{0}, \ldots, e_{n}$. Then we have $u^{-2}=x^{0} e_{0}$ and $u^{-1}=\sum_{i=1}^{n} x^{i} e_{i}$, and we see that the differential system $\hat{D}$ on $\mathrm{m}$ is defined by the equation

$$
d x^{0}-\frac{1}{2} \sum_{i=1}^{r}\left(x^{i} d x^{r+i}-x^{r+i} d x^{i}\right)=0 .
$$

The Darboux's theorem shows that any regular differential system $D^{\prime}$ of dimension $n$ and of class $r$ on a manifold $M^{\prime}$ of dimension $n+1$ is locally isomorphic with the differential system $\hat{D}$ on $\mathfrak{m}$.

(2) The case where $\mu=3, \operatorname{dim} \mathfrak{g}^{-3}=2, \operatorname{dim} \mathfrak{g}^{-2}=1$ and $\operatorname{dim} \mathfrak{g}^{-1}=2$ 
(cf. 1.2, Example (2)). There is a base $e_{1}, \ldots, e_{5}$ of $m$ such that $e_{1}$, $e_{2}$ (resp. $e_{3} ;$ resp. $\left.e_{4}, e_{5}\right)$ forms a base of $\mathfrak{g}^{-3}$ (resp. $\mathfrak{g}^{-2}$, resp. $\mathfrak{g}^{-1}$ ) and such that $e_{1}=\left[e_{3}, e_{4}\right], e_{2}=\left[e_{3}, e_{5}\right]$ and $e_{3}=\left[e_{4}, e_{5}\right]$. Let $x^{1}, \ldots, x^{5}$ denote the corresponding coordinate system of $n$. Then we have $u^{-3}$ $=x^{1} e_{1}+x^{2} e_{2}, u^{-2}=x^{3} e_{3}$ and $u^{-1}=x^{4} e_{4}+x^{5} e_{5}$, and if we put $\hat{x}^{1}=x^{1}$ $+\frac{2}{3} x^{3} x^{4}-\frac{1}{6}-\left(x^{4}\right)^{2} x^{5}$ and $\hat{x}^{2}=x^{2}+\frac{2}{3} x^{3} x^{5}+\frac{1}{6} x^{4}\left(x^{5}\right)^{2}$, then we see that the differential system $\hat{D}$ on $\|$ is defined by the equations

$$
\begin{aligned}
& d \hat{x}^{1}-\left(x^{3}-\frac{1}{2} x^{4} x^{5}\right) d x^{4}=0 \\
& d \hat{x}^{2}-\left(x^{3}+\frac{1}{2} x^{4} x^{5}\right) d x^{5}=0, \\
& d x^{3}-\frac{1}{2}\left(x^{4} d x^{5}-x^{5} d x^{4}\right)=0
\end{aligned}
$$

(cf. $[1]$, p. 977).

\section{§3. The universal fundamental graded algebras}

3.1. In this section, vector spaces and Lie algebras to be considered are those over a fixed field $K$ of characteristic 0 unless otherwise specified. Let $\left(V^{p}\right)_{p \in A}$ be a family of finite dimensional vector spaces, where $A$ is a subset of $\boldsymbol{Z}$. Set $V=\sum_{p} V^{p}$ and consider the second exterior space $\wedge^{2} V$ of $V$. Denote by $V^{r} \wedge V^{s}$ the subspace of $\wedge^{2} V$ spanned by all the elements of the form $X^{r} \wedge Y^{s}$, where $X^{r} \in V^{r}, Y^{s}$ $\in V^{s}$. Then we have: $V^{r} \wedge V^{r} \cong \wedge^{2}\left(V^{r}\right) ; V^{r} \wedge V^{s}=V^{s} \wedge V^{r} \cong V^{r} \otimes V^{s}$ $(r \neq s) ; \wedge^{2}(V)=\sum V_{r \leqq s} V^{r} \wedge V^{s}$ (direct sum). Moreover, for each $X \in V$, we denote by $X^{p}$ the $V^{p}$-component of $X$ in the decomposition $V=\sum_{p} V^{p}$.

Let $m=\sum_{p 0} \mathfrak{g}^{p}$ be a graded algebra which satisfies $\operatorname{dim} \mathfrak{g}^{-1}<\infty$ and $\mathfrak{g}^{p-1}=\left[\mathfrak{g}^{p}, \mathfrak{g}^{-1}\right](p<0)$. (We do not necessarily assume $\operatorname{dim} \mathfrak{m}<\infty$.) We set $\mathscr{T}^{p}=\operatorname{Hom}\left(\sum_{r+s=p} \mathfrak{g}^{r} \wedge \mathfrak{g}^{s}, \mathfrak{g}^{p}\right)$ and $\mathscr{T}=\sum_{p \leqq-2} \mathscr{T}^{p}$, which is a subspace of $\mathscr{B}=\operatorname{Hom}\left(\wedge^{2} \mathrm{~m}, \mathfrak{n}\right)$. Now the bracket operation of the Lie algebra $\mathrm{m}$ gives an element $B$ of $\mathscr{B}$ by defining $B(X \wedge Y)=[X, Y]$ for all $X, Y$ $\in$ ul. We clearly have $B \in \mathscr{T}$. The Jacobi identity in the Lie algebra 
nt means $\mathfrak{S} B(B(X \wedge Y) \wedge Z)=0$ for all $X, Y, Z \in n$, where $\mathfrak{S}$ stands for the cyclic sum with respect to $X, Y, Z$. It follows that

$$
\underset{(X, Y, Z)}{\Im} \sum_{r+s=p} \sum_{u+v=r} B^{p}\left(B^{r}\left(X^{u} \wedge Y^{v}\right) \wedge Z^{s}\right)=0
$$

for all $X, Y, Z \in m$ and $p \leqq-2$.

Let $V$ be a finite dimensional vector space. Assume that $\operatorname{dim} V$ $\geqq 2$. By using the vector space $V$, we shall construct a graded algebra $b(V)=\sum_{p<0} b^{p}(V)$.

Let us define vector spaces $b^{p}(V)(p<0)$ and linear mappings $\bar{B}^{p}$ : $\sum_{r+s=p} b^{r}(V) \wedge b^{s}(V) \rightarrow b^{p}(V)(p \leqq-2)$ inductively as follows: First, we define $b^{-1}(V)$ to be $V$ and $b^{-2}(V)$ to be $\wedge^{2} V$. Furthermore we define $\bar{B}^{-2}$ to be the identity transformation of $\wedge^{2} V . q$ being an integer $\leqq-3$, suppose that we have defined vector spaces $b^{p}(V)(q<p<0)$ and linear mappings $\bar{B}^{p}: \sum_{r+s=p} b^{r}(V) \wedge b^{s}(V) \rightarrow b^{p}(V)(q<p \leqq-2)$ in such a way that $\bar{B}^{p}$ is surjective and its kernel $\bar{A}^{p}(V)$ is spanned by

$$
\underset{(X, Y, Z)}{\mathfrak{S}} \sum_{r+s=p} \sum_{u+v=r} \bar{B}^{r}\left(X^{u} \wedge Y^{v}\right) \wedge Z^{s} \quad\left(X, Y, Z \in \sum_{r=p+1}^{-1} b^{r}(V)\right)
$$

Let $\bar{A}^{q}(V)$ denote the subspace of $\sum_{r+s=q} b^{r}(V) \wedge b^{s}(V)$ spanned by (3.1) with $p=q$. Then we define $b^{q}(V)$ to be the factor space $\left(\sum_{r+s=q} b^{r}(V) \wedge\right.$ $\left.b^{s}(V)\right) / \bar{A}^{q}(V)$, and $\bar{B}^{q}$ to be the projection of $\sum_{r+s=p} b^{r}(V) \wedge b^{s}(V)$ onto $b^{q}(V)$, completing our inductive definition.

We put $b(V)=\sum_{p<0} b^{p}(V)$ and define a bracket operation $[$,$] in$ $b(V)$ by

$$
[X, Y]=\sum_{p \leq-2} \sum_{r+s=p} \bar{B}^{p}\left(X^{r} \wedge Y^{s}\right)
$$

for all $X, Y \in b(V)$. Then we see from (3.1) that this bracket operation in $b(V)$ satisfies the Jacobi identity. It is clear that $b(V)$ is a graded algebra. We have clearly $b^{p}(V)=\sum_{r+s=p}\left[b^{r}(V), b^{s}(V)\right]$, which means that $b^{p-1}(V)=\left[b^{p}(V), b^{-1}(V)\right]$. If $\operatorname{dim} V=n$, we have $\operatorname{dim} b^{-2}(V)$ 
$=\frac{1}{2} n(n-1), \operatorname{dim} b^{-3}(V)=\frac{1}{3}(n+1) n(n-1)$, etc..

Proposition 3.1. Let $\amalg=\sum_{p<0} \mathfrak{g}^{p}$ be a graded algebra which satisfies $\mathfrak{g}^{p-1}=\left[\mathfrak{g}^{p}, \mathfrak{g}^{-1}\right](p<0)$ and $\operatorname{dim} \mathfrak{g}^{-1} \leqq \operatorname{dim} V$. Then every linear mapping $f$ of $V$ onto $\mathrm{g}^{-1}$ is extended to a unique homomorphism $\tilde{f}$ of the graded algebra $b(V)$ onto the graded algebra $\mathrm{m}$.

This is easy from the construction of $b(V)$.

We now assert that $b^{p}(V) \neq\{0\}$ for any $p<0$. Indeed, we can easily construct a graded algebra $\mathrm{m}=\sum_{p<0} \mathfrak{g}^{p}$ such that $\operatorname{dim} \mathfrak{g}^{p}=1(p<-1)$ and $\operatorname{dim} \mathfrak{g}^{-1}=2$ and such that $\mathfrak{g}^{p-1}=\left[\mathfrak{g}^{p}, \mathfrak{g}^{-1}\right](p<0)$. Theorefore by Prop. 3.1, we have $\operatorname{dim} b^{p}(V) \geqq 1$ for any $p<0$, proving our assertion.

Let $\mu$ be an integer $>0$. Since $\sum_{p<-\mu} b^{p}(V)$ is a graded ideal of $b(V)$, we see that the factor space $b(V, \mu)=b(V) / \sum_{p<-\mu} b^{p}(V)$ becomes a fundamental graded algebra of $\mu$-th kind.

By Prop. 3.1, we get

Proposition 3.2. Let $\mathrm{n}=\sum_{p=-\mu}^{-1} \mathrm{~g}^{p}$ be a fundamental graded algebra of $\mu$-th kind. Assume that $\operatorname{dim} \mathfrak{g}^{-1} \leqq \operatorname{dim} V$. Then every linear mapping $f$ of $V$ onto $\mathfrak{g}^{-1}$ is extended to a unique homomorphism $\tilde{f}$ of the graded algebra $b(V, \mu)$ onto the graded algebra $\mathrm{M}$.

The graded algebra $b(V, \ell)$ is called a universal fundamental graded algebra of $\mu$-th kind.

Corollary 1. Let $\mathfrak{u}=\sum_{p=-\mu}^{-1} \mathrm{~g}^{p}$ be a fundamental graded algebra of $\mu$-th kind. Assume that $\operatorname{dim} \mathfrak{g}^{p}=\operatorname{dim} b^{p}\left(\mathfrak{g}^{-1}\right)(-\mu \leqq p \leqq-1)$.

(1) The graded algebra $\mathfrak{n}$ is isomorphic with the graded algebra $b\left(\mathfrak{g}^{-1}, \mu\right)$.

(2) Let $G^{0}(\mathrm{n})$ be the group of all the automorphisms of the graded algebra nt. Then the mapping $G^{0}(\mathrm{~m}) \ni a \rightarrow a \mid \mathrm{g}^{-1}$ (the restriction of $a$ to $\left.\mathrm{g}^{-1}\right) \in G L\left(\mathrm{~g}^{-1}\right)$ gives an isomorphism of the group $G^{0}(\mathrm{~m})$ onto the group $G L\left(\mathrm{~g}^{-1}\right)$.

Corollary 2. Let $D$ be a regular differential system of dimension 
$n$ and of $\mu$-th kind on a manifold $M$. Then we have

$$
\operatorname{dim} \mathfrak{g}^{p}(x) \leqq \operatorname{dim} b^{p}\left(\boldsymbol{R}^{n}\right)(-\mu \leqq p \leqq-1, x \in M) .
$$

Corollary 3. D being as in Cor. 3, assume that

$$
\operatorname{dim} \mathfrak{g}^{p}(x)=\operatorname{dim} b^{p}\left(\boldsymbol{R}^{n}\right)(-\mu \leqq p \leqq-1, x \in M) .
$$

Then the regular differential system $D$ is of type $b\left(\boldsymbol{R}^{n}, \mu\right)$.

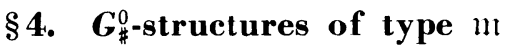

4.1. In this section, we shall consider a fixed fundamental graded algebra $\mathfrak{m}=\sum_{p<0} \mathrm{~g}^{p}$ of $\ell$-th kind over $\boldsymbol{R}$. We put $m=\operatorname{dim} m$.

$\mathfrak{D}^{p}$ being as in $\S 2$, denote by $H^{0}$ the subgroup of $G L(\mathrm{~m})$

consisting of all the elements a such that $a \mathrm{D}^{p}=\mathrm{b}^{p}$ for any $p<0$. Denote by $S^{0}$ the subgroup of $H^{0}$ consisting of all the elements a such that $a \mathrm{~g}^{p}=\mathrm{g}^{p}$ for any $p<0$, and by $N^{0}$ the (normal) subgroup of $H^{0}$ consisting of all the elements a such that $a X^{p} \equiv X^{p}\left(\bmod \mathfrak{D}^{p+1}\right)$ for any $X^{p} \in \mathrm{g}^{p}$ and $p<0$, where we put $\mathfrak{b}^{0}=\{0\}$. We have $H^{0}=S^{0} \cdot N^{0}$.

Denote by $\mathscr{B} *$ the subset of $\mathscr{B}=\operatorname{Hom}\left(\wedge^{2} m, \mathrm{~m}\right)$ consisting of all the elements $B$ such that the vector space $\|$ together with the family $\left(\mathfrak{g}^{p}\right)_{p<0}$ gives a fundamental graded algebra with $B$ as bracket operation. Let $B \in \mathscr{B}, a \in H^{0}$, and express $a$ as $a^{\prime} \cdot a^{\prime \prime}\left(a^{\prime} \in S^{0}, a^{\prime \prime} \in N^{0}\right)$. Then we define an element $B^{a}$ of $\mathscr{B}$ by $B^{a}(X \wedge Y)=a^{\prime-1} B\left(\left(a^{\prime} X\right) \wedge\left(a^{\prime} Y\right)\right)$ for all $X, Y \in m$. We have $\left(B^{a}\right)^{b}=B^{a b}$. Thus the group $H^{0}$ linearly acts on $\mathscr{B}$ to the right. $\mathscr{B} *$ is clearly an invariant set of $\mathscr{B}$.

Let $(F, \omega)$ be a $H^{0}$-structure ${ }^{1)}$ on a manifold $M$ of dimension $m$. Let $\pi$ denote the projection of $F$ onto $M$. Every element $z \in F$ gives a linear isomorphism of $m$ onto $T_{x}(M)$, where $x=\pi(z)$. $\omega$ being an $\mathfrak{m}$-valued 1 -form on $F$ (the basic form of $F$ ), we have $\pi_{*} X=z \cdot \omega(X)$ for any $X \in T_{z}(F)$ and $z \in F$. We denote by $\omega^{p}$ the $\mathfrak{g}^{p}$-component of $\omega$

1) For the definition of a $G$-structure, we refer to $[13]$, $\$ 1$. 
in the decomposition $m=\sum_{p<0} \mathrm{~g}^{p}$.

Let $D$ be a regular differential system on a manifold $M$ of dimension $m$ satisfying the following condition:

$$
\operatorname{dim} \mathfrak{g}^{p}(x)=\operatorname{dim} \mathfrak{g}^{p}(p<0, x \in M) .
$$

Note that $D^{-\mu}=T(M)$ under this condition. As is easily observed, $D$ gives rise to an $H^{0}$-structure $(F, \omega)$ on $M$ (unique up to equivalence) such that the differential system $\pi^{*} D^{p}$ is defined by the equations $\omega^{r}$ $=0(r<p)$ for any $p<0$. (We have only to define $F$ to be the totality of the linear isomorphisms $z$ of $n$ onto $T_{x}(M)$ satisfying $z \cdot \mathrm{D}^{p}=D^{p}(x)$, where $x$ runs over $M$.)

We have easily

Proposition 4.1. The assignment $(M, D) \rightarrow(F, \omega)$ is compatible with the respective isomorphisms.

Let $D$ be a regular differential system on a manifold $M$ of dimension $m$ satisfying condition $(4.1)$, and let $(F, \omega)$ be the corresponding $H^{0}$-structure on $M$. Let $z \in F$ and set $x=\pi(z)$. We have $z \cdot \triangleright^{p}=D^{p}(x)$, and define a linear isomorphism $\alpha(z)$ of $\mathrm{nt}$ onto $\mathrm{m}(x)$ by $\alpha(z) \cdot X^{p}$ $=\alpha^{p}\left(z X^{p}\right)$ for any $X^{p} \in \mathrm{g}^{p}$ and $p<0$. Let $a \in H^{0}$ and express it as $a^{\prime} \cdot a^{\prime \prime}\left(a^{\prime} \in S^{0}, a^{\prime \prime} \in N^{0}\right)$. Then we have clearly $\alpha(z a)=\alpha(z) a^{\prime}$. By using $\alpha$, we now define a mapping $T$ of $F$ to $\mathscr{B} *$ by

$$
T(z)(X \wedge Y)=\alpha(z)^{-1}[\alpha(z) X, \alpha(z) Y]
$$

for any $z \in F$ and $X, Y \in n$. We have

$$
T(z a)=T(z)^{a}
$$

for any $z \in F$ and $a \in H^{0}$. For any $p \leqq-2$, let $I(p)$ denote the subset of $\boldsymbol{Z} \times \boldsymbol{Z}$ consisting of all the pairs $(r, s)$ such that $r+s<p$ and $p<r$, $s<0$. If $(r, s) \in I(p)$, we have $r, s \leqq-2$.

Proposition 4.2. For any $p \leqq-2$, we have 


$$
\begin{aligned}
\Omega^{p}= & d \omega^{p}+\frac{1}{2} \sum_{r+s * p} T\left(\omega^{r} \wedge \omega_{s}\right) \equiv 0^{2)} \\
& \left\{\bmod \omega^{r}(r \leqq p) ; \omega^{r} \wedge \omega^{s}((r, s) \in I(p))\right\} .
\end{aligned}
$$

Proof. We may assume without loss of generality that the principal fiber bundle $F$ is trivial. Take a cross-section $g$ of $M$ to $F$, and set $\xi=g^{*} \omega$ and $\xi^{p}=g^{*} \omega^{p}$. Since $\pi^{*} D^{p}$ is defined by the equations $\omega^{r}=0(r<p)$, we see that $D^{p}$ is defined by the equations $\xi^{r}=0(r<p)$. Let $x \in M$ and set $z=g(x)$. We have $U=\pi * g * U=z \cdot \xi(U)$ for any $U \in T_{x}(M)$. Therefore we have $U \equiv z \xi^{p}(U)\left(\bmod D^{p+1}(x)\right)$ for any $U$ $\in D^{p}(x)$, whence

$$
\alpha^{p}(U)=\alpha(z) \cdot \xi^{p}(U)
$$

Let $U \in D^{r}(x)$ and $V \in D^{s}(x)$. We assert that

$$
\left(g^{*} \Omega^{p}\right)(U \wedge V)=0 \quad \text { if } r+s \geqq p
$$

Indeed, we can find an $\underline{X}_{x} \in \underline{D}^{r}(x)$ (resp. $\left.a \underline{Y}_{x} \in \underline{D}^{s}(x)\right)$ such that $U=X_{x}$ (resp. $V=Y_{x}$ ). We have

$$
\begin{gathered}
\left(g^{*} \Omega^{p}\right)(U \wedge V)=U \xi^{p}(Y)-V^{p}(x)-\xi^{p}\left([X, Y]_{x}\right) \\
+\sum_{u+v=p} T(z)\left(\xi^{u}(U) \wedge \xi^{v}(V)\right) .
\end{gathered}
$$

2) For the notations, we refer to [13], $\S 1$ : For each $p<0$, take a base $\left(e_{i}^{p}\right)_{1 \leqq i \aleph_{n} p}$ of $\mathrm{g}^{p}$ and let $\left(\omega_{i}^{p}\right)$ be the corresponding expression of the $\mathrm{g}^{p}$-valued 1 -form $\omega^{p}$ on $F$. Then we have:

$$
\begin{aligned}
& \omega^{r} \wedge \omega^{s}=\sum_{i, j} \omega_{i}^{r} \wedge \omega_{j}^{s} e_{i}^{r} \wedge e_{j}^{s}, \\
& T\left(\omega^{r} \wedge \omega^{s}\right)=\sum_{i, j, k} T_{i j k}\left(\omega_{i}^{r} \wedge \omega_{j}^{s} e_{k}^{r+s},\right. \\
& d \omega^{p}==\sum_{i} d \omega_{i}^{p} e_{i}^{p},
\end{aligned}
$$

where $\left(T_{i j k}(z)\right)_{1 \leqq k \leqq n^{r+s}}$ is the expression of the $\mathfrak{g}^{r+s}$-valued function $T(z)\left(e_{i}^{r} \wedge e_{j}^{s}\right)$ on $F$ with respect to the base $\left(e_{k}^{r+s}\right)$. Now let $\left(\Omega_{i}^{p}\right)$ be the expression of the $\mathfrak{g}^{p}$. valued 2-form $\Omega^{p}$ on $F$ with respect to the base $\left(e_{i}^{p}\right)$. Then the equality " $\Omega^{p}$ $\equiv 0\{\bmod \cdots\} "$ in Prop. 4.2 means that

$$
\begin{aligned}
\Omega_{i}^{p} & \equiv\left\{\bmod \omega_{j}^{r}\left(r \leqq p, 1 \leqq j \leqq n^{r}\right) ; \omega_{j}^{r} \wedge \omega_{k}^{s}\right. \\
& \left.\left((r, s) \in I(p), 1 \leqq j \leqq n^{r}, 1 \leqq k \leqq n^{s}\right)\right\} \quad\left(1 \leqq i \leqq n^{p}\right) .
\end{aligned}
$$


If $r+s>p$, we have clearly $\left(g^{*} \Omega^{p}\right)(U \wedge V)=0$. Now suppose that $r+s=p$. Then by using (4.2) and (4.4), we have

$$
\begin{aligned}
\left(g^{*} \Omega^{p}\right)(U \wedge V)= & -\xi^{p}\left([X, Y]_{x}\right)+T(z)\left(\xi^{r}(U) \wedge \xi^{s}(V)\right) \\
= & -\alpha(z)^{-1} \cdot \alpha^{p}\left([X, Y]_{x}\right) \\
& +\alpha(z)^{-1}\left[\alpha(z) \cdot \xi^{r}(U), \alpha(z) \cdot \xi^{s}(V)\right] \\
= & -\alpha(z)^{-1} \cdot\left[\alpha^{r}(U), \alpha^{s}(V)\right]+\alpha(z)^{-1} \cdot\left[\alpha^{r}(U), \alpha^{s}(V)\right] \\
= & 0 .
\end{aligned}
$$

Thus we have proved our assertion. (4.5) means that

$$
g^{*} \Omega^{p} \equiv 0\left\{\bmod \xi^{r} \wedge \xi^{s}(r+s<p)\right\}
$$

Since $z$ and $g \circ \pi(z)(z \in F)$ lie in the same fiber of $F$, we can find an element $a(z) \in H^{0}$ such that $z=g \circ \pi(z) \cdot a(z)^{-1}$. Moreover $a(z)$ can be expressed as $a^{\prime}(z) \cdot a^{\prime \prime}(z)\left(a^{\prime}(z) \in S^{0}, a^{\prime \prime}(z) \in N^{0}\right)$. Setting $\xi=\pi^{*} \xi$, then we have $\omega=a \cdot \xi$, whence

$$
\omega^{p} \equiv a^{\prime} \cdot \bar{\xi}^{p}\left\{\bmod \xi^{r}(r<p)\right\}
$$

and

$$
\bar{\xi}^{p} \equiv a^{\prime-1} \cdot \omega^{p}\left\{\bmod \omega^{r}(r<p)\right\}
$$

By (4.7), we have

$$
d \omega^{p} \equiv a^{\prime} \cdot d \bar{\xi}^{p}\left\{\bmod \xi^{r}(r \leqq p) ; d \xi^{r}(r<p)\right\},
$$

and by (4.6),

$$
d \xi^{r} \equiv 0\left\{\bmod \xi^{u} \wedge \xi^{v}(u+v \leqq r)\right\} .
$$

It follows from $\left(4.7^{\prime}\right)$ that

$$
d \omega^{p} \equiv a^{\prime} d \xi^{p}\left\{\bmod \omega^{r}(r \leqq p) ; \omega^{r} \wedge \omega^{s}(r+s<p)\right\}
$$


Since $T(z)^{a^{\prime}(z)}=T(g \circ \pi(z))$, it follows from $\left(4.7^{\prime}\right)$ that

$$
a^{\prime} \cdot d \xi^{p} \equiv-\frac{1}{2} \sum_{r+s=p} T\left(\omega^{r} \wedge \omega^{s}\right)\left\{\bmod \omega^{r} \wedge \omega^{s}(r+s<p)\right\}
$$

From (4.8) and (4.9), we get the desired equalities. We have thereby proved Prop. 4.2 .

Remark. We set $\mathscr{T}_{*}^{p}=\operatorname{Hom}\left(\sum_{(r, s) \in I(p)} \mathrm{g}^{r} \wedge \mathfrak{g}^{s}, \mathfrak{g}^{p}\right)$, and $\mathscr{T}_{*}=\sum_{p \leqq-2} \mathscr{T}_{*}^{p}$ which is a subspace $\mathscr{B}$. By Prop. 4.2 , we can find, for each $p \leqq-2$, a unique mapping $T_{*}^{p}$ of $F$ to $\mathscr{T}_{*}^{p}$ satisfying the following equality:

$$
\begin{gathered}
d \omega^{p}+\frac{1}{2} \sum_{r+s=p} T\left(\omega^{r} \wedge \omega^{s}\right)+\frac{1}{2} \sum_{(r, s) \in I(p)} T_{*}^{p}\left(\omega^{r} \wedge \omega^{s}\right) \equiv 0 \\
\left\{\bmod \omega^{r}(r \leqq p)\right\} .
\end{gathered}
$$

We set $T_{*}=\sum_{p \leqq-2} T_{*}^{p}$. Then the sum $T+T_{*}$ may be considered as the structure function of the $H^{0}$-structure $(F, \omega)$.

We now state the following proposition without proof, which is a converse of Prop. 4.2.

Proposition 4.3. Let $(F, \omega)$ be a $H^{0}$-structure on a manifold $M$. Assume that there is a mapping $T$ of $F$ to $\mathscr{B}_{*}$ satisfying the equalities in Prop. 4.2. (It is clear that $T$ is uniquley determined by this condition.) Then there is a unique regular differential system $D$ on $M$ satisfying condition (4.1) such that the given $(F, \omega)$ is equivalent to the $H^{0}$-structure associated with $D$.

We have thus characterized regular differential systems satisfying condition (4.1) in terms of $H^{0}$-structures.

4.2. The group $H^{0}$ acted on $\mathscr{B}_{*}$ to the right. We denote this transformation group $H^{0}$ on $\mathscr{B}_{*}$ by $\left(\mathscr{B}_{*}, H^{0}\right)$. The bracket operation in the fundamental graded algebra $\mathfrak{m}$ determines an element $B_{0}$ in $\mathscr{B}_{*}$. Let $G_{\#}^{0}(\mathfrak{M})$ denote the isotropy group of $\left(\mathscr{B}_{*}, H^{0}\right)$ at the point $B_{0} \in \mathscr{B}_{*}$. We set $G^{0}(\mathrm{~m})=G_{\#}^{0}(\mathrm{~m}) \cap S^{0}$. Then we have $N^{0} C G_{\#}^{0}(\mathfrak{m})$ and $G_{\#}^{0}(\mathrm{~m})$ $=G^{0}(\mathrm{~m}) \cdot N^{0}$. The group $G^{0}(\mathrm{nt})$ is nothing but the group of all the auto- 
morphisms of the graded algebra 11 .

We shall characterize regular differential systems of type $m$ in terms of $G_{\#}^{0}(\mathrm{~m})$-structures.

Let $D$ be a regular differential system on a manifold $M$ of dimension $m$ satisfying condition (4.1), and let $(F, \omega)$ be the corresponding $H^{0}$-structure on $M$. Consider the image $T(F)$ of $F$ by the mapping $T$ : $F \rightarrow \mathscr{B}_{*}$. By (4.3), we see that $T(F)$ is $H^{0}$-invariant. Let $\left[B_{0}\right]$ denote the orbit through the point $B_{0} \in \mathscr{B}_{*}$ of $\left(\mathscr{B}_{*}, H\right)$. Then by using (4.3), we can easily show that $T(F)=\left[B_{0}\right]$ if and only if the regular differential system $D$ on $M$ is of type 11 .

Assume that $D$ is of type IIt. Let $P_{\#}$ be the subset of $F$ consisting of all the elements $z$ with $T(z)=B_{0}$. Since $T(F)=\left[B_{0}\right]$, we see from (4.3) that $P_{\sharp}$ is a $G_{\sharp}^{0}(\mathrm{nI})$-subbundle of $F$. Thus we get a $G_{\sharp}^{0}(\mathrm{~m})$ structure $\left(P_{\#}, \omega\right)$ on $M$. The equalities in Prop. 4.2 restricted to $P_{\#}$ yield the following equalities:

$$
\begin{aligned}
& d \omega^{p}+\frac{1}{2} \sum_{r+s=p}\left[\omega^{r}, \omega^{s}\right] \equiv 0 \\
& \left\{\bmod \omega^{r}(r \leqq p) ; \omega^{r} \wedge \omega^{s}((r, s) \in I(p))\right\}(p \leqq-2) .
\end{aligned}
$$

Thus we have shown that to every regular differential system $D$ of type $m$ on a manifold $M$ of dimension $m$ there is associated a $G_{\#}^{0}(n 1)$. structure $\left(P_{\#}, \omega\right)$ on $M$ satisfying equalities (4.10). By Prop. 4.1, it is clear that the assignment $(M, D) \rightarrow\left(P_{\sharp}, \omega\right)$ is compatible with the respective isomorphisms. Conversely, let $\left(P_{\#}, \omega\right)$ be a $G_{\#}^{0}(n t)$-structure on a manifold $M$ satisfying equalities (4.10). Then it is clear from Prop. 4.3 that there is a unique regular differential system $D$ of type mt on $M$ such that the given $\left(P_{\#}, \omega\right)$ is equivalent to the $G_{\sharp}^{0}(n)$-structure associated with $D$.

4.3. We shall now generalize the notion of a regular differential system of type $m$ on a manifold of dimension $m$. Let $G^{0}$ be a Lie subgroup of the group $G^{0}(\mathrm{~m})$ of all the automorphisms of the graded algebra $\mathrm{n}$. Then the product $G_{\#}^{0}=G^{0} \cdot N^{0}$ is clearly a Lie subgroup of 
From (4.6), we get

$$
a^{\prime} \cdot d \xi^{p} \equiv-\frac{1}{2} \sum_{r+s=p} a^{\prime} \cdot\left(\pi^{*} g^{*} T\right)\left(\xi^{r} \wedge \xi^{s}\right)\left\{\bmod \xi^{r} \wedge \xi^{s}(r+s<p)\right\}
$$

the group $G_{\#}^{0}(n \mathrm{II})=G^{0}(\mathrm{nIt}) \cdot N^{0}$.

Definition 4.1. We say that a $G_{\sharp}^{0}$-structure $\left(P_{\#}, \omega\right)$ on a manifold $M$ is of type $m$ if it satisfies equalities (4.10).

The above argument shows that giving a $G_{\#}^{0}(m i)$-structure of type $m$ on a manifold $M$ of dimension $m$ is nothing but giving a regular differential system of type $n$ on $M$. Moreover, $G_{\#}^{0}$ being a Lie subgroup of $G_{\#}^{0}(\mathrm{~m})$, we know that every $G_{\sharp}^{0}$-structure $\left(P_{\#}, \omega\right)$ of type $\mathbb{m}$ on a manifold $M$ gives rise to a $G_{\#}^{0}(m)$-structure of type $m$ on $M$ in such a way that the given $P_{\#}$ is a $G_{\#}^{0}$-subbundle of the $G_{\# \text {-bundle. }}^{0}$

4.4. Finally we shall generalize the notion of the standard differential system of type $m$ to give the notion of the standard $G_{\sharp \text {-structure }}^{0}$ of type $\mathrm{mt}$.

Let $D$ be the standard differential system of type $m$ on the manifold $M(\mathfrak{m})$, and let $(F, \omega)$ be the corresponding $H^{0}$-structure on $M(\mathfrak{m})$. At each $x \in M(\mathfrak{n t})$, we define a linear isomorphism of $\mathrm{nt}$ onto $T_{x}(M(\mathfrak{m l}))$ by $g(x) \cdot X=X_{x}$ for all $X \in \mathrm{n}$. It is clear that the mapping $x \rightarrow g(x)$ gives a cross-section $g$ of the principal fiber bundle $F$. Moreover we have clearly $T(g(x))=B_{0}$. Let $\bar{P}_{\#}$ be the $G_{\#}^{0}(n t)$-subbundle of $F$ defined by $T=B_{0}$. Then it follows that $g$ gives a cross-section of $\bar{P}_{\# \text {. Now }}$ let $G^{0}$ and $G_{\#}^{0}$ be as above. We denote by $P_{\#}$ the subset of $\bar{P}_{\#}$ consisting of all the elements of the form $g(x) \cdot a\left(x \in M(\mathfrak{m}), a \in G_{\sharp}^{0}\right)$, which is a $G_{\#}^{0}$-subbundle of $\bar{P}_{\#}$. Thus we get a $G_{\#}^{0}$-structure $\left(P_{\#}, \omega\right)$ of type

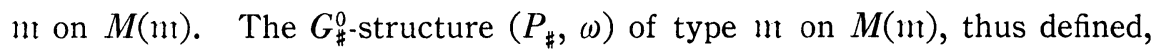
is called the standard $G_{\sharp}^{0}$-structure of type $\mathrm{m}$.

\section{$\S 5$. The algebraic prolongations}

5.1. Let $\mathfrak{m}=\sum_{p<0} \mathrm{~g}^{p}$ be a fundamental graded algebra over a field $K$ of characteristic $0 .{ }^{p}$ We shall construct a graded algebra $\mathrm{g}(\mathrm{m})=\sum_{\boldsymbol{p} \in \boldsymbol{Z}} \mathrm{g}^{p}(\mathrm{~m})$ 
over $K$ satisfying the following conditions:

1) The graded subalgebra $\sum_{p<0} \mathfrak{g}^{p}(n 1)$ of $\mathfrak{g}(n 1)$ coincides with the given fundamental graded algebra $\mathrm{m}$;

2) Let $p$ be any integer $\supseteq 0$. If $X^{p} \in \mathfrak{g}^{p}(\mathfrak{n t})$ and if $\left[X^{p}, \mathfrak{g}^{-1}\right]$ $=\{0\}$, then $X^{p}=0$;

3) $\mathfrak{g}(\mathrm{mt})$ is maximum among graded algebras satisfying condition 1) and 2) above. More precisely, let $\mathfrak{h}=\sum_{p} \mathfrak{h}^{p}$ be any graded algebra satisfying 1) and 2). Then $\mathfrak{h}$ is imbedded in $\mathfrak{g}(\mathrm{nI})$ as graded subalgebra.

We set $\mathfrak{g}^{p}=\mathfrak{g}^{p}(\mathfrak{m}) \quad(p<0)$. Let us define vector spaces $\mathfrak{g}^{p}(\mathfrak{m})$ $(p \geqq 0)$ and bilinear mappings $\Phi^{p, r}: \mathfrak{g}^{p}(n 1) \times \mathfrak{g}^{r} \ni\left(X^{p}, Y^{r}\right) \rightarrow\left[X^{p}, Y^{r}\right]$ $\in \mathrm{g}^{p+r}(\mathrm{nt})(p \geqq 0, r<0)$ inductively as follows:

First $\mathrm{g}^{0}(\mathrm{~m})$ is defined to be the Lie algebra of all the derivations $X^{0}$ of the graded algebra $m$, and $\Phi^{0, r}$ is defined by $\left[X^{0}, Y^{r}\right]=X^{0} Y^{r}$ for any $X^{0} \in \mathrm{g}^{0}(\mathrm{~m})$ and $Y^{r} \in \mathrm{g}^{r} . \quad k$ being an integer $>0$, suppose that we have defined vector spaces $\mathfrak{g}^{p}(\mathfrak{n})(0 \leqq p<k)$ and bilinear mappings $\Phi^{p, r}$ $(0 \leqq p<k, r<0)$ in such a way that

$$
\left[\left[X^{p}, Y^{-1}\right], Z^{r}\right]-\left[\left[X^{p}, Z^{r}\right], Y^{-1}\right]-\left[X^{p},\left[Y^{-1}, Z^{r}\right]\right]=0
$$

for any $X^{p} \in \mathfrak{g}^{p}(\mathrm{~m}), Y^{-1} \in \mathfrak{g}^{-1}$ and $Z^{r} \in \mathfrak{g}^{r}(0 \leqq p<k, r<0)$. Then we put $\mathfrak{q}^{k}(\mathfrak{m})=\sum_{r<0} \operatorname{Hom}\left(\mathfrak{g}^{r}, \mathfrak{g}^{r+k}(\mathfrak{m})\right)$, which may be identified with a subspace of Hom $\left(\mathfrak{m}, \sum_{p<k} \mathfrak{g}^{p}(\mathfrak{n})\right)$. This being said, $\mathrm{g}^{k}(\mathfrak{m})$ is defined to be the subspace of $\mathfrak{q}^{k}(\mathrm{~m})$ consisting of all the elements $X^{k}$ satisfying the following equalities:

$$
\left[X^{k}\left(Y^{-1}\right), Z^{r}\right]-\left[X^{k}\left(Z^{r}\right), Y^{-1}\right]-X^{k}\left(\left[Y^{-1}, Z^{r}\right]\right)=0
$$

for any $Y^{-1} \in \mathfrak{g}^{-1}$ and $Z^{r} \in \mathfrak{g}^{r}(r<0)$, and $\Phi^{k, r}$ is defined by $\left[X^{k}, Y^{r}\right]$ $=X^{k}\left(Y^{r}\right)$ for any $Y^{r} \in \mathrm{g}^{r}$. Then we have clearly equalities (5.1) with $p=k$. We have thus completed our inductive definition.

By using the fact that $\mathfrak{g}^{r-1}=\left[\mathfrak{g}^{r}, \mathfrak{g}^{-1}\right](r<0)$, we can easily prove property 2) for $\mathfrak{g}(\mathfrak{m})$ and the following equalities:

$$
\left[\left[X^{p}, Y^{r}\right], Z^{s}\right]-\left[\left[X^{p}, Z^{s}\right], Y^{r}\right]-\left[X^{p},\left[Y^{r}, Z^{s}\right]\right]=0
$$


On differential systems, graded Lie algebras and pseudo-groups

for any $X^{p} \in \mathfrak{g}^{p}(\mathrm{nI}), \quad Y^{r} \in \mathfrak{g}^{r}, Z^{s} \in \mathfrak{g}^{s}(p \geqq 0, r, s<0)$.

We set $\left[Y^{r}, Y^{p}\right]=-\left[X^{p}, Y^{r}\right]$ for any $X^{p} \in \mathfrak{g}^{p}(n)$ and $Y^{r} \in \mathfrak{g}^{r}$ $(p \geqq 0, r<0)$. Let us now define bilinear mappings

$$
\Phi^{p, q}: \mathfrak{g}^{p}(\mathrm{~m}) \times \mathrm{g}^{q}(\mathrm{~m}) \ni\left(X^{p,} Y^{q}\right) \rightarrow\left[X^{p}, Y^{q}\right] \in \mathrm{g}^{p+q}(\mathrm{~m} \mathrm{t})(p, q \geqq 0)
$$

inductively as follows: First, $\Phi^{0,0}$ is defined to be the bracket operation in the Lie algebra $\mathfrak{g}^{0}(111)$, i.e., $\left[X^{0}, Y^{0}\right]=X^{0} Y^{0}-Y^{0} X^{0}$ for any $X^{0}, Y^{0}$ $\in \mathrm{g}^{0}(\mathrm{~m}) . \quad k$ being an integer $>0$, suppose that we have defined bilinear mappings $\Phi^{p, q}(p, q \geq 0, p+q<k)$ in such a way that

$$
\left[\left[X^{p}, Y^{q}\right], Z^{r}\right]-\left[\left[X^{p}, Z^{r}\right], Y^{q}\right]-\left[X^{p},\left[Y^{q}, Z^{r}\right]\right]=0
$$

for any $X^{p} \in \mathfrak{g}^{p}(\mathrm{~m}), \quad Y^{q} \in \mathfrak{g}^{q}(\mathrm{~m})$ and $Z^{r} \in \mathfrak{g}^{r}(p, q \geqq 0, p+q<k, r<0)$. Let $p, q$ be any integers with $p, q \geqq 0$ and $p+q=k$, and take any $X^{p}$ $\in \mathfrak{g}^{p}(\mathrm{nt})$ and $Y^{q} \in \mathrm{g}^{q}(\mathrm{~m} \mathrm{t})$. Define an element $W^{k}$ of $\mathfrak{q}^{k}(\mathrm{nt})$ by

$$
W^{k}\left(Z^{r}\right)=\left[\left[X^{p}, Z^{r}\right], Y^{q}\right]+\left[X^{p},\left[Y^{q}, Z^{r}\right]\right]
$$

for any $Z^{r} \in \mathfrak{g}^{r}(r<0)$. Then we can easily show that $W^{k} \in \mathfrak{g}^{k}(\mathfrak{m})$. This being said, we put $\left[X^{p}, Y^{q}\right]=W^{k}$. The bilinear mapping $\Phi^{p, q}$, thus defined, clearly satisfies (5.3). Thus we have completed our inductive definition.

We have clearly $\left[X^{p}, Y^{q}\right]=-\left[Y^{q}, X^{p}\right]$ for any $X^{p} \in \mathfrak{g}^{p}(\mathfrak{m})$ and $Y^{q} \in \mathrm{g}^{q}(\mathrm{~m})(p, q \geqq 0)$. Moreover we can prove the equalities:

$$
\left[\left[X^{p}, Y^{q}\right], Z^{r}\right]+\left[\left[Y^{q}, Z^{r}\right], X^{p}\right]+\left[\left[Z^{r}, X^{p}\right], Y^{q}\right]=0
$$

for any $X^{p} \in \mathrm{g}^{p}(\mathrm{il}), Y^{q} \in \mathrm{g}^{q}(\mathrm{n}), Z^{r} \in \mathrm{g}^{r}(\mathrm{\prime \prime \prime})(p, q, r \geqq 0)$.

Finally we put $\mathfrak{g}(n i)=\sum_{p} \mathfrak{g}^{p}(n)$. Then we know that the bilinear mappings $\Phi^{p, q}$ together with the bracket operation in $n$ define a structure of graded algebra in $\mathfrak{g}(\mathrm{mI})$ so that $\mathfrak{g}(\mathrm{mi})$ has the desired properties. The graded algebra $\mathfrak{g}(\mathrm{mi})$ is called the prolongation of the fundamental graded algebra $m$.

5.2. Let $\mathrm{m}$ and $\mathfrak{g}(\mathrm{m} \mathrm{I})$ be as above. Suppose that we are given a 
sequence $\mathfrak{g}^{0}, \ldots, \mathfrak{g}^{k}$ which satisfies the following conditions:

1) $\mathfrak{g}^{p}$ is a subspace of $\mathfrak{g}^{p}(n \mathrm{nt})(0 \leqq p \leqq k)$;

2) The family $\left(\mathfrak{g}^{p}\right)_{-\alpha<p \leqq k}$ satisfies $\left[\mathfrak{g}^{r}, \mathfrak{g}^{s}\right] C \mathfrak{g}^{r+s}(r+s \leqq k)$. Then we define a sequence $\mathfrak{g}^{k+1}, \mathfrak{g}^{k+2}, \ldots$ inductively as follows: $l$ being an integer $>k$, suppose that we have defined $\mathfrak{g}^{k+1}, \ldots, \mathfrak{g}^{l-1}$ as subspaces of $\mathfrak{g}^{k+1}(\mathrm{nt}), \cdots, \mathfrak{g}^{l-1}(\mathrm{nIt})$ respectively, in such a way that $\left[\mathfrak{g}^{p}, \mathfrak{g}^{r}\right] \subset \mathfrak{g}^{p+r}$ $(k<p<l, r<0)$. Then we define $\mathfrak{g}^{l}$ to be the subspace of $\mathfrak{g}^{l}(\mathfrak{l l t})$ consisting of all the elements $X^{l}$ such that $\left[X^{l}, \mathfrak{g}^{-1}\right] C \mathfrak{g}^{l-1}$ or equivalently $\left[X^{l}\right.$, $\left.\mathfrak{g}^{r}\right] \subset \mathfrak{g}^{l+r}(r<0)$. If we put $\mathfrak{g}=\sum_{p \in \boldsymbol{Z}} \mathfrak{g}^{p}$, then we can easily prove $\mathfrak{g}$ to be a graded subalgebra of $\mathfrak{g}(\mathrm{mi})$. The graded algebra $\mathfrak{g}$ is called the prolongation of $\left(n, \mathfrak{g}^{0}, \cdots, \mathfrak{g}^{k}\right)$. We put $\mathfrak{q}^{l}=\sum_{r<0} \operatorname{Hom}\left(\mathfrak{g}^{r}, \mathfrak{g}^{r+l}\right)$, being a subspace of $q^{l}(m)$. Then we have $\mathfrak{g}^{l} \subset q^{l} \cap \mathfrak{g}^{l}(n)$ for any $l$. Moreover we have $\mathfrak{g}^{l}=\mathfrak{q}^{l} \cap \mathfrak{g}^{l}(\mathrm{nII})$ for any $l>k$.

Let $\mathfrak{g}^{0}$ be a subalgebra of $\mathfrak{g}^{0}(\mathfrak{n})$. Then we have clearly $\left[\mathfrak{g}^{r}, \mathfrak{g}^{s}\right]$ $\mathrm{Cg}^{r+s}(r+s \leqq 0)$. Therefore we may talk about the prolongation of $\left(n 1 \mathrm{t}, \mathfrak{g}^{0}\right)$.

Definition 5.1. Let $u$ be a fundamental graded algebra.

(1) $m$ is called of finite type (resp. of infinite type) if $\operatorname{dim} \mathfrak{g}(\mathfrak{n})$ $<\infty($ resp. $=\infty)$.

(2) Let $\mathfrak{g}^{0}, \ldots, \mathfrak{g}^{k}$ be a sequence satisfying condition (5.4) and let $\mathfrak{g}$ be the prolongation of $\left(n \mathfrak{l}, \mathfrak{g}^{0}, \ldots, \mathfrak{g}^{k}\right)$. Then $\left(n \mathfrak{l}, \mathfrak{g}^{0}, \ldots, \mathfrak{g}^{k}\right)$ is called of finite type (resp. of infinite type) if $\operatorname{dim} \mathfrak{g}<\infty$ (resp. $=\infty)$.

5.3. Examples. Let $\|=\sum_{p<0} \mathfrak{g}^{p}$ be a fundamental graded algebra of $\mu$-th kind over $\boldsymbol{R}$. We shall investigate, for several special cases of $m$, the prolongation $\mathfrak{g}(n i)=\sum_{p} \mathfrak{g}^{p}(n i)$ as well as the group $G^{0}(n i)$ of all the automorphisms of the graded algebra 11 . First we make some general remarks on $\mathfrak{g}(n \mathrm{ut})$ and $G^{0}(\mathrm{nt})$. i) For each $\lambda \in \boldsymbol{R}$, define a linear automorphism $\varepsilon(\lambda)$ of $n$ by $\varepsilon(\lambda) X^{p}=\lambda^{p} x^{p}$ for any $X^{p} \in \mathfrak{g}^{p}(p<0)$. Then $\varepsilon(\lambda)$ is in the center of $G^{0}(\mathfrak{m})$. ii) $\mathrm{g}^{0}(\mathrm{~m})$, being the Lie algebra of $G^{0}(\mathrm{~m})$, contains a (unique) element $E$ in its center such that $\left[E, X^{p}\right]$ $=p X^{p}$ for any $X^{p} \in \mathfrak{g}^{p}(p<0)$. iii) Let $\mathfrak{h}^{p}(p \geqq-1)$ denote the subspace 
of $\mathfrak{g}^{p}(\mathrm{nI})$ consisting of all the elements $X^{p}$ such that $\left[X^{p}, \mathfrak{g}^{r}\right]=\{0\}$ for any $r<-1$. Then we have $\left[\mathfrak{h}^{p}, \mathfrak{g}^{-1}\right] \subset \mathfrak{h}^{p-1}(p \geqq 0)$. Moreover, $\mathfrak{h}^{0}$ may be identified with a subalgebra of $\mathfrak{g l}^{l}\left(\mathfrak{g}^{-1}\right)$, and from the construction of $g(m)$, we know that $\mathfrak{h}^{p}(p>0)$ may be identified with the $p$-th prolongation $\left(\mathfrak{h}^{0}\right)^{(p)}$ of $\mathfrak{h}^{0}$ in the usual sense.

(1) The case where $\mu=2$ and $\operatorname{dim} \mathfrak{g}^{-2}=1$ (cf. 1.2, Example (1); 2.3, Example (1); [13], §7). We put $n=\operatorname{dim} \mathfrak{g}^{-1}$. Let $e_{0}$ be a base of $\mathrm{g}^{-2}$, and let $B$ be the anti-symmetric bilinear form on $\mathrm{s}^{-1}$ defined by $\left[X^{-1}, Y^{-1}\right]=B\left(X^{-1}, Y^{-1}\right) e_{0}\left(X^{-1}, Y^{-1} \in \mathfrak{g}^{-1}\right)$. Considering a base $e_{1}$, $\ldots, e_{n}$ of $\mathfrak{g}^{-1}$ as was explained in 2.3, Example (1), we define a matrix $I=\left(I_{i j}\right)$ of degree $n$ by $I_{i j}=B\left(e_{i}, e_{j}\right)$. Then the group $G^{0}(n t)$ may be represented, with respect to the base $e_{0}, \ldots, e_{n}$ of $n$, by matrices of degree $n+1$ of the form:

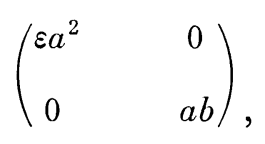

where $a>0, b \in G L(n, \boldsymbol{R}), \varepsilon^{2}=1$ and ${ }^{t} b I b=\varepsilon I$. Let us now study the graded algebra $\mathfrak{g}(\mathrm{m})$. Let $\delta^{p}$ denote the linear mapping $\mathfrak{g}^{p}(\mathrm{~m}) \ni X^{p} \rightarrow$ $\left[X^{p}, e_{0}\right] \in \mathfrak{g}^{p-2}(\mathrm{~m})$. Then the subspace $\mathfrak{h}^{p}$ of $\mathfrak{g}^{p}(\mathrm{nt})$ clearly coincides with the kernel of $\delta^{p}(p \geqq-1)$. We assert that the subalgebra $\mathfrak{h}^{0}$ of $\mathfrak{g l}\left(\mathfrak{g}^{-1}\right)$ is involutive. Indeed, let $n$ be of class $r$. Then we see that $\mathfrak{h}^{0}$ may be represented by matrices of degree $n$ of the form:

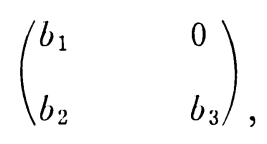

where $b_{2}, b_{3}$ are arbitrary and $b_{1} \in \mathfrak{w}(r, \boldsymbol{R})$. Since $\mathfrak{s}^{\prime}(r, \boldsymbol{R})$ is involutive, it follows that the same holds for $\mathfrak{h}^{0}$, proving our assertion.

Proposition 5.1. $\delta^{p}$ is surjective for any $p$. Therefore we have $\mathfrak{g}^{p-2}(\mathfrak{m} \mathfrak{t}) \cong \mathfrak{g}^{p}(\mathfrak{m}) / \mathfrak{h}^{p}$.

Proof. $\delta^{p}$ is clearly surjective for any $p<0 . \delta^{0}$ in surjective, because $\delta^{0}(E)=-2 e_{0}$. Let us prove $\delta^{1}$ to be surjective. Let $X^{-1}$ be 
any element of $\mathfrak{g}^{-1}$. Define an element $u \in \operatorname{Hom}\left(\mathfrak{g}^{-2}, \mathfrak{g}^{-1}\right)$ by $u\left(e_{0}\right)$ $=X^{-1}$, and an element $b \in \operatorname{Hom}\left(\mathfrak{g}^{-1}, \mathfrak{g l}\left(\mathfrak{g}^{-1}\right)\right)$ by

$$
b\left(Y^{-1}\right) Z^{-1}=\frac{1}{2} B\left(X^{-1}, Z^{-1}\right) Y^{-1}+\frac{1}{2} B\left(Y^{-1}, Z^{-1}\right) X^{-1}
$$

for any $Y^{-1}, Z^{-1} \in \mathfrak{g}^{-1}$. This being said, define an element $w \in \operatorname{Hom}\left(\mathfrak{g}^{-1}\right.$, $\left.\mathfrak{g}^{0}(m)\right)$ by

$$
w\left(Y^{-1}\right)=-\frac{1}{2} B\left(X^{-1}, Y^{-1}\right) E+b\left(Y^{-1}\right)
$$

for any $Y^{-1} \in \mathfrak{g}^{-1}$, and set $X^{1}=u+w$, being an element of $\mathfrak{q}^{1}(m)$ $=\operatorname{Hom}\left(\mathfrak{g}^{-2}, \mathfrak{g}^{-1}(\mathfrak{n t})\right)+\operatorname{Hom}\left(\mathfrak{g}^{-1}, \mathfrak{g}^{0}(\mathfrak{m})\right)$. Then we can easily show $X^{1}$ $\in \mathrm{g}^{1}(\mathrm{~m})$. We have clearly $\delta^{1}\left(X^{1}\right)=u\left(e_{0}\right)=X^{-1}$. Therefore we have proved $\delta^{1}$ to be surjective. (We here explain the Spencer cohomology groups associated with the subalgebra $\mathfrak{h}^{0}$ of $\mathrm{gl}\left(\mathfrak{g}^{-1}\right)$, which will be needed from now on. Set $C^{p, q}=\mathfrak{h}^{p-1} \otimes \wedge^{q}\left(\mathfrak{g}^{-1}\right)^{*}(p, q \geqq 0)$, and define an operator $\partial: C^{p, q} \rightarrow C^{p-1, q+1}$ by

$$
(\partial c)\left(X_{1} \wedge \cdots \wedge X_{q+1}\right)=\sum_{i=1}^{q+1}(-1)^{i}\left[c\left(X_{1} \wedge \cdots \wedge \hat{X}_{i} \wedge \cdots \wedge X_{q+1}\right), X_{i}\right]
$$

for any $c \in C^{p, q}$ and $X_{1}, \ldots, X_{q+1} \in \mathfrak{g}^{-1}$. Then we have $\partial^{2}=0$. The cohomology groups $H^{p, q}(p, q \geqq 0)$ associated with the complex $\left\{C^{p, q}\right\}$ with operator $\partial$ are called the Spencer cohomology groups. Since $\mathfrak{h}^{0}$ is involutive, we have $H^{p, q}=\{0\}$ for any $p>0$ and $q \geqq 0([9])$.) Now, $l$ being an integer $>0$, suppose that $\delta^{l}$ is surjective. Let $X^{l-1}$ be any element of $\mathfrak{g}^{l-1}(\mathrm{nI})$. Define an element $u \in \operatorname{Hom}\left(\mathfrak{g}^{-2}, \mathfrak{g}^{l-1}(n 1)\right)$ by $u\left(e_{0}\right)$ $=X^{l-1}$. Since $\delta^{l}$ is surjective, we can find an element $\bar{w} \in \operatorname{Hom}\left(\mathfrak{g}^{-1}\right.$, $\left.\mathfrak{g}^{l}(\mathfrak{n t})\right)$ such that $\left[u\left(e_{0}\right), Z^{-1}\right]=\left[\bar{w}\left(Z^{-1}\right), e_{0}\right]$ for any $Z^{-1} \in \mathfrak{g}^{-1}$. Define an element $c \in \mathrm{g}^{l-1}(\mathrm{~m}) \otimes \wedge^{2}\left(\mathrm{~g}^{-1}\right) *$ by

$$
c\left(Y^{-1} \wedge Z^{-1}\right)=\left[\bar{w}\left(Y^{-1}\right), Z^{-1}\right]-\left[\bar{w}\left(Z^{-1}\right), Y^{-1}\right]-u\left(\left[Y^{-1}, Z^{-1}\right]\right)
$$

for any $Y^{-1}, Z^{-1} \in \mathfrak{g}^{-1}$. Then we see easily that $c\left(Y^{-1} \wedge Z^{-1}\right) \in \mathfrak{h}^{l-1}$, 
whence $c \in C^{l, 2}=\mathfrak{h}^{l-1} \otimes \wedge^{2}\left(\mathfrak{g}^{-1}\right)^{*}$. Moreover we have $\partial c=0$. Since $H^{l, 2}$ $=\{0\}$, it follows that there is an element $b \in C^{l+1,1}=\mathfrak{h}^{l} \otimes\left(\mathfrak{g}^{-1}\right)^{*}$ such that $c=-\partial b$. Set $w=\bar{w}+b$, being an element of $\operatorname{Hom}\left(\mathfrak{g}^{-1}, \mathfrak{g}^{l}(m)\right)$, and set $X^{l+1}=u+w$, being an element of $\mathfrak{q}^{l+1}(\mathfrak{m})=\operatorname{Hom}\left(\mathfrak{g}^{-2}, \mathfrak{g}^{l-1}(\mathrm{nt})\right)$ $+\operatorname{Hom}\left(\mathfrak{g}^{-1}, \mathfrak{g}^{l}(\mathfrak{n l})\right)$. Then we can easily show $X^{l+1} \in \mathfrak{g}^{l+1}(\mathfrak{n l})$. We have clearly $\delta^{l+1}\left(X^{l+1}\right)=u\left(e_{0}\right)=X^{l-1}$. We have thus proved $\delta^{l+1}$ to be surjective. By induction, we have thereby completed the proof of Prop. 5.1 .

$\left(1^{\prime}\right)$ Let $m$ be as in (1). From the proof of Prop. 5.1, we know that there is a (unique) injective linear mapping $\Phi^{1}$ of $\mathfrak{g}^{-1}$ to $\mathfrak{g}^{1}(\mathfrak{m})$ satisfying the following equalities:

$$
\begin{aligned}
& {\left[\Phi^{1}\left(X^{-1}\right), e_{0}\right]=X^{-1},} \\
& {\left[\left[\Phi^{1}\left(X^{-1}\right), Y^{-1}\right], Z^{-1}\right]=\frac{1}{2} B\left(X^{-1}, Y^{-1}\right) Z^{-1}} \\
& \quad+\frac{1}{2} B\left(X^{-1}, Z^{-1}\right) Y^{-1}+\frac{1}{2} B\left(Y^{-1}, Z^{-1}\right) X^{-1}
\end{aligned}
$$

for any $X^{-1}, Y^{-1}, Z^{-1} \in \mathfrak{g}^{-1}$. Then it can be proved that $\left[X^{0}, \Phi^{1}\left(X^{-1}\right)\right]$ $=\Phi^{1}\left(\left[X^{0}, X^{-1}\right]\right)$ for any $X^{0} \in \mathfrak{g}^{0}(\mathfrak{n t})$ and $X^{-1} \in \mathfrak{g}^{-1}$. If we put $\mathfrak{g}^{0}$ $=\mathfrak{g}^{0}(\mathfrak{m})$ and $\mathfrak{g}^{1}=\Phi^{1}\left(\mathfrak{g}^{-1}\right)$, then it follows that the sequence $\mathfrak{g}^{0}, \mathfrak{g}^{1}$ satisfies condition (5.4). We denote by $\mathfrak{g}=\sum_{p} \mathfrak{g}^{p}$ the prolongation of $\left(\mathfrak{m}, \mathfrak{g}^{0}, \mathfrak{g}^{1}\right)$. Now, assume that $\mathfrak{m}$ is non-degenerate. Then it can be shown that $\mathfrak{g}^{p}$ $=\{0\}(p>2)$ and $\operatorname{dim} \mathrm{g}^{2}=1$. More precisely, $\mathrm{g}^{2}$ has a base $X^{2}$ defined by $\left[X^{2}, e_{0}\right]=-E$ and $\left[X^{2}, Y^{-1}\right]=\Phi^{1}\left(Y^{-1}\right)$ for any $Y^{-1} \in \mathrm{g}^{-1}$. Furthermore we see that $\mathfrak{g}$ is isomorphic with the simple Lie algebra $\mathfrak{s p}(k+1, \boldsymbol{R})$, where $k=\frac{n}{2}$. The graded algebra $g$ is known as the projective contact algebra (cf. [9]).

(2) The case where $\mu=3, \operatorname{dim} g^{-3}=2, \operatorname{dim} g^{-2}=1$ and $\operatorname{dim} \mathfrak{g}^{-1}=2$, i.e., $\mathrm{m} \cong b\left(\boldsymbol{R}^{2}, 3\right)$ as graded algebras (cf. 1.2, Example (2); 2.3, Example (2); [1]). Consider a base $e_{1}, \ldots, e_{5}$ of $m$ as was explained in $\S 2$, Example (2). Then the group $G^{0}(\mathrm{~m})$ may be represented, with respect 
to this base, by matrices of degree 5 of the form:

$$
\left(\begin{array}{ccccc}
\triangle a & \triangle c & 0 & 0 & 0 \\
\triangle b & \triangle d & 0 & 0 & 0 \\
0 & 0 & \triangle & 0 & 0 \\
0 & 0 & 0 & a & c \\
0 & 0 & 0 & b & d
\end{array}\right),
$$

where $\triangle=a d-b c \neq 0$. We have $g^{p}(m)=\{0\}(p>3)$, and we have the natural identifications as follows: $\mathfrak{g}^{0}(\mathfrak{m})=\mathfrak{g}^{-1} \otimes\left(\mathfrak{g}^{-1}\right)^{*}$ and $\mathfrak{g}^{p}(\mathrm{~m})$ $=\left(\mathfrak{g}^{-p}\right) *(0<p \leqq 3)$. Moreover $\mathfrak{g}(\mathrm{nt})$ is isomorphic with the exceptional simple Lie algebra of dimension 14.

As for other examples, see the previous paper [13].

\section{§6. Infinitesimal automorphisms of the standard differential system of type $n$}

6.1. Throughout this section, we shall consider a fixed fundamental graded algebra $m=\sum_{p<0} \mathfrak{g}^{p}$ of $\mu$-th kind over $\boldsymbol{R}$. Let $\mathfrak{g}(\mathfrak{m})=\sum_{p \in Z} \mathfrak{g}^{p}(\mathrm{nt})$ denote the prolongation of $u$, and $M(n)$ the simply connected Lie group whose Lie algebra is given by $n$.

Let $D$ be the standard differential system of type $\mathfrak{m}$ on $M(\mathrm{~m})$. We denote by $\mathscr{A}$ the sheaf of all the local infinitesimal automorphisms of $(M(\mathrm{n}), D)$, which is a transitive $L A S$ on $M(\mathrm{n})$ ). (For the definition of an $L A S$, see [9].) Let $\left(P^{\sharp}, \omega\right)$ be the $G_{\sharp}^{0}(m)$-structure on $M(m)$ corresponding to $D$. Then $\mathscr{A}$ is nothing but the sheaf of all the local infinitesimal automorphisms of $\left(P_{\#}, \omega\right)$. The sheaf $\mathscr{A}$ is called the standard Lie algebra sheaf of type $\mathrm{nt}$. Now let $\xi$ be the Maurer-Cartan form of the Lie group $M(u 1)$. Since $d \xi+\frac{1}{2}[\xi, \xi]=0$, then we have the following equalities:

$$
d \xi^{p}+\frac{1}{2} \sum_{r+s=p}\left[\xi^{r}, \xi^{s}\right]=0 \quad(p<0),
$$


$\xi^{p}$ being the $\mathfrak{g}^{p}$-component of $\xi$ in the decomposition $m=\sum_{p<0} \mathfrak{g}^{p}$.

Lemma 6.1. Let $X$ be a vector field on an open set $U$ of $M(\mathrm{~m})$. Then $X$ gives a local cross-section of $\mathscr{A}$ if and only if there is a family $\left(f^{b}\right)_{p \leqq 0}$ of functions satisfying the following conditions:

1) $f^{p}$ is a $\mathrm{g}^{p}(\mathrm{~m})$-valued function on $U(p \leqq 0)$;

2) $f^{p}=\xi^{p}(X)(p<0)$;

3) $d f^{p} \equiv \sum_{r=p}^{-1}\left[f^{p-r}, \xi^{r}\right]\left\{\bmod \xi^{r}(r<p)\right\} \quad(p \leqq 0)$.

Moreover the family $\left(f^{p}\right)_{p \leqq 0}$ as above is uniquely determined.

Proof. Let $\mathfrak{g}_{\#}^{0}(\mathfrak{m})\left(\right.$ resp. $\left.\mathfrak{n}^{0}\right)$ denote the Lie algebra of $G_{\#}^{0}(\mathfrak{n})$ (resp. $\left.N^{0}\right)$. Then we have $\mathfrak{g}_{\sharp}^{0}(\mathfrak{n})=\mathfrak{g}_{\sharp}^{0}(\mathfrak{m})+\mathfrak{l}^{0}$ (direct sum) and we see that $\mathfrak{n}^{0}$ is equal to the subalgebra of $\mathfrak{g l}(\mathfrak{n t})$ consisting of all the elements $A$ such that $A D^{p} \subset D^{p+1}$ for any $p<0$. Let us consider the cross-section $g$

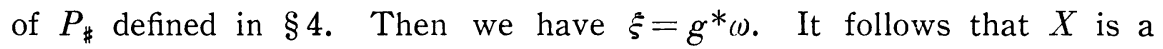
local cross-section of $\mathscr{A}$ (or induces a local infinitesimal automorphism of $\left.\left(P_{\sharp}, \omega\right)\right)$ if and only if there is $a \mathfrak{g}_{\sharp}^{0}(\mathfrak{m})$-valued function $B$ on $U$ such that $L_{X} \xi=B \xi$ or equivalently there is a $\mathfrak{g}^{0}(\mathfrak{m})$-valued function $f^{0}$ on $U$ such that

$$
L_{X} \xi^{p} \equiv\left[f^{0}, \xi^{p}\right]\left\{\bmod \xi^{r}(r<p)\right\} \quad(p<0) .
$$

Now put $\left.f^{p}=\xi^{p}(X)=X\right\lrcorner \xi^{p}(p<0)$, which is a $\mathrm{g}^{p}$-valued function on $U$. Then we have $\left.\left.L_{X} \xi^{p}=d(X\lrcorner \xi^{p}\right)+X\right\lrcorner d \xi^{p}$. From (6.1), it follows that

$$
L_{X} \xi^{p}=d f^{p}-\sum_{r=p+1}^{-1}\left[f^{p-r}, \xi^{r}\right] \quad(p<0) .
$$

Lemma 6.1 is immediate from these arguments.

Lemma 6.2. Let $X$ be a local cross-section of $\mathscr{A}$ defined on an open set $U$ of $M(\mathfrak{m})$. Then there is a unique family $\left(f^{p}\right)_{p \in \boldsymbol{Z}}$ of functions satisfying the following conditions: 
1) $f^{p}$ is a $\mathrm{g}^{p}(\mathrm{~m})$-valued function on $U(p \in \boldsymbol{Z})$;

2) $f^{p}=\xi^{p}(X) \quad(p<0)$;

3) $d f^{p}=\sum_{r<0}\left[f^{p-r}, \xi^{r}\right](p \in \boldsymbol{Z})$.

Proof. $k$ being an integer $\geqq 1$, suppose that there is a family $\left(f^{p}\right)_{p<k}$ of functions satisfying the following conditions:

$\left.1^{\prime}\right) \quad f^{p}$ is a $\mathrm{g}^{p}(\mathrm{~m})$-valued function on $U(p<k)$;

$\left.2^{\prime}\right) \quad f^{p}=\xi^{p}(X) \quad(p<0)$;

$\left.3^{\prime}\right) \quad d f^{p} \equiv \sum_{r=p-k+1}^{-1}\left[f^{p-r}, \xi^{r}\right]\left\{\bmod \xi^{r}(r \leqq p-k)\right\} \quad(p<k)$.

(Note that, in the case when $k=1$, the family $\left(f^{p}\right)_{p<1}$ in Lemma 6.1 satisfies these conditions.) Then by $\left.3^{\prime}\right)$, we can find a $q^{k}(m)$-valued function $f^{k}$ on $U$ such that

$$
\begin{aligned}
d f^{p} \equiv \sum_{r=p-k+1}^{-1}\left[f^{p-r}, \xi^{r}\right]+f^{k}\left(\xi^{p-k}\right) & \\
& \left\{\bmod \xi^{r}(r \leqq p-k-1)\right\} \quad(p<k) .
\end{aligned}
$$

By applying the exterior differentiation $d$ to the both sides of (6.3), we get

$$
\begin{array}{r}
\sum_{r=p-k+1}^{-1}\left[d f^{p-r}, \xi^{r}\right]+f^{k}\left(d \xi^{p-k}\right) \equiv 0 \\
\left\{\bmod \xi^{r}(r \leqq p-k) ; d \xi^{r}(r \neq p-k)\right\} .
\end{array}
$$

We have

$$
\begin{aligned}
& d f^{p-r} \equiv f^{k}\left(\xi^{p-r-k}\right)\left\{\bmod \xi^{s}(s \neq p-r-k)\right\}, \\
& d \xi^{r} \equiv 0\left\{\bmod \xi^{s} \wedge \xi^{t}(s+t=r)\right\}, \\
& d \xi^{p-k}+\frac{1}{2} \sum_{r=p-k+1}^{-1}\left[\xi^{r}, \xi^{p-r-k}\right]=0 .
\end{aligned}
$$


It follows that

$$
\begin{array}{r}
\sum_{r=p-k+1}^{-1}\left[f^{k}\left(\xi^{p-r-k}\right), \xi^{r}\right]-\frac{1}{2} \sum_{r=p-k+1}^{-1} f^{k}\left(\left[\xi^{r}, \xi^{p-r-k}\right]\right) \equiv 0 \\
\left\{\bmod \xi^{r}(r \leqq p-k) ; \xi^{r} \wedge \xi^{s}(r+s \neq p-k)\right\} .
\end{array}
$$

" $\equiv 0 "$ in this last equalities clearly reduces to " $=0 "$. Therefore we get

$$
\left[f^{k}\left(\xi^{r}\right), \xi^{s}\right]+\left[f^{k}\left(\xi^{s}\right), \xi^{r}\right]-f^{k}\left(\left[\xi^{r}, \xi^{s}\right]\right)=0 \quad(r, s<0),
$$

whence

$$
\left[f^{k}\left(X^{r}\right), Y^{s}\right]-\left[f^{k}\left(Y^{s}\right), X^{r}\right]-f^{k}\left(\left[X^{r}, Y^{s}\right]\right)=0
$$

for any $X^{r} \in \mathfrak{g}^{r}, Y^{s} \in \mathfrak{g}^{s}(r, s<0)$. This means that $f^{k}$ is a $\mathfrak{g}^{k}(\mathfrak{m})$-valued function on $U$, and by $(6.3)$, we have

$$
d f^{p} \equiv \sum_{r=p-k}^{-1}\left[f^{p-r}, \xi^{r}\right]\left\{\bmod \xi^{r}(r \leqq p-k-1)\right\} \quad(p<k+1) .
$$

Thus the family $\left(f^{p}\right)_{p<k+1}$ satisfies conditions $\left.\left.1^{\prime}\right), 2^{\prime}\right)$ and $\left.3^{\prime}\right)$ with $k$ replaced by $k+1$. By induction, we have thereby obtained a family $\left(f^{b}\right)_{p \in \boldsymbol{Z}}$ of functions which satisfies conditions 1), 2) and $\left.3^{\prime}\right)$ for all $k \geqq 1$. Let $p$ be any integer and take an integer $k \geqq 0$ with $p<k-\mu$. Then by $3^{\prime}$ ), we have $d f^{p}=\sum_{r<0}\left[f^{p-r}, \xi^{r}\right]$, because $\xi^{r}=0$ if $r<-\mu$. Thus we have proved existence of a family $\left(f^{p}\right)_{p \in Z}$ satisfying conditions 1), 2) and 3). Uniqueness is clear.

We shall denote by $\left(f_{X}^{p}\right)_{p \in \boldsymbol{Z}}$ the family $\left(f^{p}\right)_{p \in \boldsymbol{Z}}$ in Lemma 6.2.

Lemma 6.3. Let $x$ be any point of $M(\mathrm{MI})$ and let $a=\sum_{p \leq k} a^{p}$ be any element of $\mathrm{g}(\mathrm{mi})$, where $a^{p} \in \mathrm{g}^{p}(\mathrm{nI})$. Then there is a unique cross. section $X$ of $\mathscr{A}$ such that

$$
\begin{array}{ll}
f_{X}^{p}(x)=a^{p} & (p \leqq k), \\
f_{X}^{p}=0 & (p>k) .
\end{array}
$$


Proof. Let $k$ be any integer $\geqq 0$. Set $\mathfrak{m}^{k}=\sum_{p=-\mu}^{k} \mathfrak{g}^{p}(\mathfrak{m})$ and denote by $u^{p}$ the projection of $\mathrm{m}^{k}$ onto $\mathrm{g}^{p}(\mathrm{~m})$. Then the system $\left(u^{p}\right)_{-\mu \leqq p \leqq k}$ defines a "linear coordinate system" of the manifold $\mathfrak{m}_{k}$. Consider the product $M(n) \times \mathfrak{n}^{k}$. Then $\xi^{p}\left(\right.$ resp. $\left.u^{p}\right)$ may be confounded with a form (resp. a function) on $M(m) \times m^{k}$. Let us consider a system of pfaffian equations as follows:

$$
\alpha^{p}=d u^{p}-\sum_{r=p-k}^{-1}\left[u^{p-r}, \xi^{r}\right]=0 \quad(-\mu \leqq p \leqq k) .
$$

We assert that system (6.4) is completely integrable. By applying the exterior differentiation $d$ to the both sides of (6.4), we have

$$
d \alpha^{p}=-\sum_{t=p-k}^{-1}\left[d u^{p-t}, \xi^{t}\right]-\sum_{r=p-k}^{-1}\left[u^{p-r}, d \xi^{r}\right] .
$$

By (6.1) and (6.4), it follows easily that

$$
d \alpha^{p}=-\sum_{t=p-k}^{-1}\left[\alpha^{p-t}, \xi^{t}\right]
$$

which proves our assertion. Now denote by $\mathscr{A}_{k}$ the vector subsheaf of $\mathscr{A}$ which consists of all the elements $\underline{X}_{x}$ such that $f_{X}^{p}=0$ for any $p>k$. From Lemmas 6.1 and 6.2 and from the fact that system (6.4) is completely integrable, we see that, at each $x \in M(\mathfrak{m})$, the linear mapping $\underline{X}_{x} \rightarrow \sum_{p=-\mu}^{k} f_{X}^{p}(x)$ maps the stalk $\mathscr{A}_{k}(x)$ isomorphically onto the vector space $\mathfrak{m}^{k}$. Since $M(\mathfrak{m})$ is simply connected, the standard argument shows that every local cross-section $X$ of $\mathscr{A}_{k}$ defined on a connected open set $U$ of $M(\mathrm{nt})$ is uniquely extended to a global cross-section $\bar{X}$ of $\mathscr{A}_{k}$. Therefore we get Lemma 6.3,

Lemma 6.4. Let $X$ and $Y$ be two cross-sections of $\mathscr{A}$ defined on a common open set $U$ of $M(\mathrm{ni})$. Then we have

$$
f_{[X, Y]}^{p}=-\sum_{r+s=p}\left[f_{X}^{r}, f_{Y}^{s}\right] \quad(p \in \boldsymbol{Z}) .
$$


Proof. By (6.1), we have

$$
f_{[X, Y]}^{p}=X f_{Y}^{p}-Y f_{X}^{p}+\sum_{r+s=p}\left[f_{X}^{r}, f_{Y}^{s}\right] \quad(p<0),
$$

and by Lemma 6.2 ,

$$
X f_{Y}^{p}=-\underset{\substack{r+s=p \\ s<0}}{ }\left[f_{X}^{r}, f_{Y}^{s},\right], Y f_{X}^{p}=\underset{\substack{r+s=p \\ r<0}}{ }\left[f_{X}^{r}, f_{Y}^{s}\right] \quad(p<0) .
$$

If we put $g^{p}=-\sum_{r+s=p}\left[f_{X}^{r}, f_{Y}^{s}\right]$, then it follows that $f_{[X, Y]}^{p}=g^{p}(p<0)$. We have

$$
d g^{p}=-\sum_{r+s=p}\left[d f_{X}^{r}, f_{Y}^{s}\right]-\sum_{r+s=p}\left[f_{X}^{r}, d f_{Y}^{s}\right] \quad(p \in Z),
$$

and by using Lemma 6.2, we get easily $d g^{p}=-\sum_{t<0}\left[g^{p-t}, \xi^{t}\right]$. Therefore we have shown that the family $\left(g^{p}\right)_{p \in \boldsymbol{Z}}$ satisfies the conditions in Lemma 6.2. Thus we get $g^{p}=f_{[X, Y]}^{p}(p \in \boldsymbol{Z})$.

Let $X$ and $Y$ be two local cross-sections of $\mathscr{A}$ defined on a common open set $U$ of $M(\mathrm{~m})$. Let $\left(\varphi_{t}\right)$ be a local one parameter group of local transformations which is generated by the vector field $X$. Then the vector fields $\varphi_{t *} Y$ also give local cross-sections of $\mathscr{A}$. We set as follows: $f_{t}^{p}=\varphi_{t}^{*}\left(f_{X}^{p}\right)$ and $g_{t}^{p}=\varphi_{t}^{*}\left(f_{\varphi_{t *}}^{p}\right)$.

Lemma 5.6. The notation being as above, we have

$$
\frac{\partial g_{t}^{p}}{\partial t}=L_{Y} f_{t}^{p}+\sum_{\substack{r+s=p \\ r, s \geqq 0}}\left[f_{t}^{r}, g_{t}^{s}\right] \quad(p \geqq 0) .
$$

Proof. Put $\xi_{t}^{p}=\varphi_{t}^{*} \xi^{p}$. Then we have $L_{X} \xi_{t}^{p}=\frac{\partial \xi_{t}^{p}}{\partial t}=\varphi_{t}^{*}\left(L_{X} \xi^{p}\right)$ and $L_{Y} \xi_{t}^{p}=\varphi_{t}^{*}\left(L_{\varphi_{t *} Y} \xi^{p}\right)$. Therefore from Lemma 6.2 and equalities (6.2) follow the following equalities:

$$
\begin{aligned}
& L_{X} \xi_{t}^{-1} \equiv\left[f_{t}^{0}, \xi_{t}^{-1}\right], L_{X} \xi_{t}^{p} \equiv 0 \quad(p \leqq-2), \\
& L_{Y} \xi_{t}^{-1} \equiv\left[g_{t}^{0}, \xi_{t}^{-1}\right], L_{Y} \xi_{t}^{p} \equiv 0 \quad(p \leqq-2),
\end{aligned}
$$




$$
d f_{t}^{p} \equiv\left[f_{t}^{p+1}, \xi_{t}^{-1}\right], d g_{t}^{p} \equiv\left[g_{t}^{p+1}, \xi_{t}^{-1}\right] \quad(p \geqq 0),
$$

where " $A \equiv B$ " means that $A \equiv B\left\{\bmod \xi_{t}^{r}(r \leqq-2)\right\}$. Lemma 6.5 can be proved by induction and by the use of these equalities. The details are left to the readers.

6.2. We shall now make a general consideration on a sheaf of Lie algebras which is subordinate to a differential system. Let $D$ be a regular differential system of type $\mathrm{m}$ on a manifold $M$ of dimension $m$, where $m=\operatorname{dim} n$. Let $\mathscr{L}$ be a sheaf of Lie algebras of local vector fields on $M$. We assume that $\mathscr{L}$ is transitive on $M$ and that it leaves invariant the differential system $D$.

We fix a point $e$ of $M$ and denote by $\mathscr{L}(e)^{0}$ the isotropy subalgebra of $\mathscr{L}(e)$. Starting from the pair $\left(\mathscr{L}(e), \mathscr{L}(e)^{0}\right)$, let us define a family $\left(\mathscr{L}(e)_{*}^{p}\right)_{p \in \boldsymbol{Z}}$ of subspaces of $\mathscr{L}(e)$ inductively as follows (cf. [9]): First, we define $\mathscr{L}(e)_{*}^{p}(p<0)$ as $\mathscr{L}(e)$ and $\mathscr{L}(e)_{*}^{0}$ as $\mathscr{L}(e)^{0} . p$ being an integer $>0$, suppose that we have defined $\mathscr{L}(e)_{*}^{p-1}$. Then $\mathscr{L}(e)_{*}^{p}$ is defined to be the subspace of $\mathscr{L}(e)_{*}^{p-1}$ consisting of all the elements $\underline{X}_{e}$ such that $\left[\underline{X}_{e}, \mathscr{L}(e)_{*}^{p-1}\right] \subset \mathscr{L}(e)_{*}^{p-1}$, completing our inductive definition. We have $\mathscr{L}(e)_{*}^{p-1} \supset \mathscr{L}(e)_{*}^{p}$ and $\left[\mathscr{L}(e)_{*}^{r}, \mathscr{L}(e)_{*}^{s}\right] \subset \mathscr{L}(e)_{*}^{r+s}$.

Denote by $\mathscr{L}(e)^{-1}$ the subspace of $\mathscr{L}(e)$ consisting of all the elements $\underline{X}_{e}$ such that $X_{e} \in D(e)$. Starting from the tripple $(\mathscr{L}(e)$, $\mathscr{L}(e)^{-1}, \mathscr{L}\left(e^{0}\right)$, let us now define a family $\left(\mathscr{L}(e)^{p}\right)_{p \in \boldsymbol{Z}}$ of subspaces of $\mathscr{L}(e)$ inductively as follows: $p$ being an integer $<-1$, suppose that we have defined $\mathscr{L}(e)^{p+1}$. Then $\mathscr{L}(e)^{p}$ is defined as $\left[\mathscr{L}(e)^{p+1}, \mathscr{L}(e)^{-1}\right]$ $+\mathscr{L}(e)^{p+1}$. $p$ being an integer $>0$, suppose now that we have defined $\mathscr{L}(e)^{p-1}$. Then $\mathscr{L}(e)^{p}$ is defined to be the subspace of $\mathscr{L}(e)^{p-1}$ consistng of all the elements $\underline{X}_{e}$ such that $\left[\underline{X}_{e}, \mathscr{L}(e)^{-1}\right] \subset \mathscr{L}(e)^{p-1}$, completing our inductive definition. We have $\mathscr{L}(e)^{p-1} \supset \mathscr{L}(e)^{p}$ and $\left[\mathscr{L}(e)^{r}\right.$, $\left.\mathscr{L}(e)^{s}\right] \subset \mathscr{L}(e)^{r+s}$.

Let $\left(D^{p}\right)_{p<0}$ be the family of differential systems associated with the regular differential system $D$. Then we have

Lemma 6.6. For any $p<0, \mathscr{L}(e)^{p}$ consists of all the elements 
$\underline{X}_{e} \in \mathscr{L}(e)$ such that $X_{e} \in D^{p}(e)$. In particular, we have $\mathscr{L}(e)^{-\mu}=\mathscr{L}(e)$.

Proof of this lemma is left to the readers.

Remark 1. We set $\mathfrak{g}^{p}(e)=\mathscr{L}(e)^{p} / \mathscr{L}(e)^{p+1} \quad$ and $\mathfrak{g}(e)=\sum_{p \in \boldsymbol{Z}} \mathfrak{g}^{p}(e)$. Since $\left[\mathscr{L}(e)^{r}, \mathscr{L}(e)^{s}\right] \subset \mathscr{L}(e)^{r+s}$, we see that $\mathrm{g}(e)$ is endowed with a structure of graded algebra. Then the graded algebra $\mathfrak{g}(e)$ may be naturally identified with a graded subalgebra of $\mathfrak{g}(\mathrm{ml})$ in such a way that $m=\sum_{p<0} \mathfrak{g}^{p}(e)$.

By using Lemma 6.6, we have easily

\section{Lemma 6.7.}

$$
\mathscr{L}(e)^{p} \supset \mathscr{L}(e)_{*}^{p} \supset \mathscr{L}(e)^{\mu p} \quad(p \geqq 0) .
$$

We set $L=\lim _{t \rightarrow \infty} \mathscr{L}(e) / \mathscr{L}(e)_{*}^{p}$, which is called the formal algebra of $\mathscr{L}(e)$ or $\mathscr{L}([9])^{p \rightarrow \infty}$ The projections of $\mathscr{L}(e)$ onto $\mathscr{L}(e) / \mathscr{L}(e)_{*}^{p}$ give rise to a homomorphism $\theta$ of the Lie algebra $\mathscr{L}(e)$ to the Lie algebra $L$. We set $L_{*}^{p}=\lim _{r \rightarrow \infty} \mathscr{L}(e)_{*}^{p} / \mathscr{L}(e)_{*}^{r}$. Then $L_{*}^{p}$ may be identified with a subspace of $L$, and we have $L=L_{*}^{-1} \supset \cdots \supset L_{*}^{p-1} \supset L_{*}^{p} \supset \cdots$ and $\bigcap_{p} L_{*}^{p}$ $=\{0\}$. We shall consider a topology in $L$ such that the family $\left(L_{*}^{p}\right)$ forms a fundamental system of neighborhoods of 0 (=the zero vector in $L)$.

6.3. Let us now return to the study of the Lie algebra sheaf $\mathscr{A}$ on $M(n 1)$. We shall apply the above argument to $\mathscr{L}=\mathscr{A}$ and the identity element $e$ of the Lie group $M(n 1)$. We denote by $A$ the formal algebra of $\mathscr{A}(e)$.

Lemma 6.8. For any $p, \mathscr{A}(c)^{p}$ consists of all the elements $\underline{X}_{c}$ $\in \mathscr{A}(e)$ such that $f_{X}^{r}(e)=0$ for any $r<p$.

This follows easily from Lemmas 6.4 and 6.6.

We set $\overline{\mathfrak{g}(\mathfrak{m})}=\prod_{p \in \boldsymbol{Z}} \mathfrak{g}^{p}(\mathfrak{m})=\prod_{p>-\mu} \mathfrak{g}^{p}(\mathfrak{m})$ (direct product). Then the structure of Lie algebra in $\mathfrak{g}(\mathrm{mt})$ yields that in $\overline{\mathfrak{g}(\mathrm{m} t)}$. The Lie algebra $\overline{\mathfrak{g}(\mathrm{m})}$ is called the formal algebra associated with the graded algebra $g(m)$. Now let $a$ be any element of $\mathfrak{g}(\mathrm{m})$. By Lemma 6.3, there is a unique 
cross-section $X$ of $\mathscr{A}$ such that $f_{X}^{p}(e)=a^{p}(p \leqq k)$ and $f_{X}^{p}=0 \quad(p>k)$. Then it follows from Lemma 6.4 that the mapping $a \rightarrow-\underline{X}_{e}$ gives an injective homomorphism $c$ of $\mathfrak{g}(111)$ to $\mathscr{A}(e)$. By lemmas 6.7 and 6.8, it is clear that $\iota$ gives rise to a homeomorphic isomorphism $\bar{\iota}$ of $\overline{\mathfrak{g}(\mathfrak{m})}$ onto $A$, where $\overline{\mathfrak{g}(\mathfrak{m})}$ should be equipped with the standard topology.

We have thereby proved the following.

Theorem 6.1. The formal algebra $A$ of the standard Lie algebra sheaf $\mathscr{A}$ of type $\mathfrak{i n}$ on $M(\mathrm{nt})$ may be naturally identified with the formal algebra $\overline{\mathrm{g}(\mathrm{nt})}$ associated with the prolongation $\mathfrak{g}(\mathrm{nI})$ of $\mathrm{nt}$.

Remark 2. Let us try to describe the subspaces $A_{*}^{p}$ of $A=\overline{\mathfrak{g}(\mathrm{m})}$ in terms of the graded algebra $\mathfrak{g}(\mathrm{m})$. Put $A^{p}=\prod_{r \geqq p} \mathrm{~g}^{r}(\mathrm{~m})$, being a subspace of $\overline{\mathrm{g}(\mathrm{m})}$. Then we have $A^{p} \supset A_{*}^{p} \supset A^{\mu p}(p \geqq 0)$ by Lemmas 6.7 and 6.8. Since $\overline{\mathfrak{g}(\mathrm{m})}=\mathrm{m}+A_{*}^{0}$ (direct sum), $A_{*}^{p}(p>0)$ consists of all the elements $X \in A_{*}^{p-1}$ such that $[X, m] \subset A_{*}^{p-1}$. Now suppose that $\mu$ $=2$ and $\operatorname{dim} \mathrm{g}^{-2}=1$, and use the notations in 5.3, Example. (1). By Prop. 5.1, we can find a linear endomorphism $\Phi$ of $g(m)$ such that $\Phi\left(\mathrm{g}^{p-2}(\mathfrak{m})\right) \subset \mathrm{g}^{p}(\mathfrak{m})$ and such that $\left[\Phi\left(X^{p-2}\right), e_{0}\right]=X^{p-2}$ for any $X^{p-2}$ $\in \mathfrak{g}^{p-2}(\mathfrak{m})$. Then we have $\mathfrak{g}^{p}(\mathfrak{m})=\Phi\left(\mathfrak{g}^{p-2}(\mathfrak{m})\right)+\mathfrak{h}^{p}$ (direct sum), and $\left[\Phi\left(X^{p-2}\right), Y^{-1}\right] \equiv \Phi\left(\left[X^{p-2}, Y^{-1}\right]\right)\left(\bmod \mathfrak{h}^{p-1}\right)$ for any $X^{p-2} \in \mathfrak{g}^{p-2}(\mathfrak{m})$ and $Y^{-1} \in \mathrm{g}^{-1}$. By using these facts, we can verify the following equalities:

$$
\begin{aligned}
& A_{*}^{0}=A^{0}, \\
& A_{*}^{1}=\mathfrak{h}^{1}+A^{2}, \\
& A_{*}^{2}=\mathfrak{h}^{2}+\left\{\mathfrak{h}^{3}+\Phi\left(\mathfrak{h}^{1}\right)\right\}+A^{4}, \\
& A_{*}^{3}=\mathfrak{h}^{3}+\left\{\mathfrak{h}^{4}+\Phi\left(\mathfrak{h}^{2}\right)\right\}+\left\{\mathfrak{h}^{5}+\Phi\left(\mathfrak{h}^{3}+\Phi\left(\mathfrak{h}^{1}\right)\right)\right\}+A^{6}, \text { etc. }
\end{aligned}
$$

If we put $\mathfrak{g}_{*}^{p}=A_{*}^{p} / A_{*}^{p+1}$, then it follows that

$$
\mathfrak{g}_{*}^{0} \cong \mathfrak{g}^{0}(\mathfrak{m})+\Phi\left(\mathfrak{g}^{-1}\right)
$$




$$
\begin{aligned}
& \mathfrak{S}_{*}^{1} \cong \mathfrak{h}^{1}+\Phi\left(\mathfrak{g}^{0}(\mathfrak{m})\right)+\Phi^{2}\left(\mathfrak{g}^{-1}\right), \\
& \mathfrak{h}_{*}^{2} \cong \mathfrak{h}^{2}+\Phi\left(\mathfrak{h}^{1}\right)+\Phi^{2}\left(\mathfrak{g}^{0}(\mathfrak{m})\right)+\Phi^{3}\left(\mathfrak{g}^{-1}\right), \text { etc. }
\end{aligned}
$$

Note that $\mathfrak{g}_{*}^{p}$ is equal to the $p$-th prolongation of $\mathfrak{g}_{*}^{0}$ and that $\mathfrak{g}_{*}^{0}$ is involutive (cf. [9]).

6.4. Suppose that we are given a sequence $\mathfrak{g}^{0}, \ldots, \mathfrak{g}^{k}$ satisfying condition (5.4), and let $\mathfrak{g}=\sum_{p \in \mathbf{Z}} \mathfrak{g}^{p}$ be the prolongation of $\left(n, \mathfrak{g}^{0}, \cdots, \mathfrak{g}^{k}\right)$. We set $\overline{\mathfrak{g}}=\prod_{p \in \boldsymbol{Z}} \mathfrak{g}^{p}$, which is a closed subalgebra of $\overline{\mathfrak{g}(n i)}$. We shall show that $\overline{\mathfrak{g}}$ may be characterized as the formal algebra $L$ of a suitable transitive Lie algebra sheaf $\mathscr{L}$ on $M(\mathrm{~m})$.

We denote by $\mathscr{L}$ the vector subsheaf of $\mathscr{A}$ consisting of all the elements $\underline{X}_{x}$ such that, for any $p(0 \leqq p \leqq k)$, the $\mathfrak{g}^{p}(\mathfrak{m})$-valued function $f_{X}^{p}$ becomes a $\mathfrak{g}^{p}$-valued function. For each $p \geqq 0$, choose a complementary subspace $\left(\mathfrak{g}^{p}\right)^{\prime}$ of $\mathfrak{g}^{p}$ in $\mathfrak{g}^{p}(\mathrm{nt})$, and denote by $\Gamma$ the projection of $\mathrm{g}^{p}(\mathrm{~m})$ onto $\left(\mathrm{g}^{p}\right)^{\prime}$. Then it is clear that $\mathscr{L}$ is composed of all $\underline{X}_{x} \in \mathscr{A}$ satisfying the following equalities:

$$
\Gamma f_{X}^{p}=0 \quad(0 \leqq p \leqq k)
$$

It should be remarked that (6.5) may be considered as a system of differential equations with $X$ as unknown.

Lemma 6.9. (1) Let $X$ be a local cross-section of $\mathscr{L}$. Then $f_{X}^{b}$ becomes a $\mathfrak{g}^{p}$-valued function for any $p \geqq 0$, or equivalently $\Gamma f_{X}^{p}=0$ $(p \geqq 0)$.

(2) Let $x \in M(\mathrm{~m})$ and $a=\sum_{p \leqq l} a^{p}$, where $a^{p} \in \mathfrak{g}^{p}$. Then there is $a$ unique cross-section $X$ of $\mathscr{L}$ such that

$$
\begin{aligned}
& f_{X}^{p}(x)=a^{p} \quad(0 \leqq p \leqq l), \\
& f_{X}^{p}=0 \quad(p>l) .
\end{aligned}
$$

(3) Let $X$ and $Y$ be two local cross-sections of $\mathscr{L}$ defined on a common open set $U$ of $M(\mathrm{~m})$, and let $\left(\varphi_{t}\right)$ be a local one parameter 
group generated by $X$. Then the vector fields $\varphi_{t_{*}} Y$ also give local cross-sections of $\mathscr{L}$.

Proof. (1) and (2) are easy. Let us prove (3) and use the notations in Lemma 6.5. Let $p$ be any integer $\geq 0$, and suppose that $\Gamma f_{\varphi_{t * Y}}^{r}=0(0 \leqq r<p)$. We have $\Gamma f_{t}^{r}=\Gamma g_{t}^{r}=0(0 \leqq r<p)$ and $\left[\mathfrak{g}^{r}, \mathfrak{g}^{s}\right]$ $\mathrm{Cg}^{r+s}$. Therefore by applying $\Gamma$ to the both sides of the equality in Lemma 6.5, we have

$$
\frac{\partial\left(\Gamma g_{t}^{p}\right)}{\partial t}=\Gamma\left[f_{t}^{0}, \Gamma g_{t}^{p}\right]
$$

Since $\Gamma g_{0}^{p}=\Gamma f_{t}^{p}=0$, we get $\Gamma g_{t}^{p}=0$ by the uniqueness theorem for systems of ordinary differential equations, whence $\Gamma f_{\varphi_{l}{ }^{*} Y}^{p}=0$. We have thereby proved (3) by induction.

By Lemma 6.9 , we know that $\mathscr{L}$ is a transitive $L A S$ on $M(n 1)$, which is called the standard Lie algebra sheaf of type $\left(n, \mathfrak{g}^{0}, \ldots, \mathfrak{g}^{k}\right)$. Let $L$ denote the formal algebra of $\mathscr{L}(e)$, which is a closed subalgebra of $A$. Then we see from Lemma 6.9 that the isomorphism $c$ of $\overline{\mathfrak{g}(\mathrm{m})}$ onto $A$ gives an isomorphism of $\overline{\mathfrak{g}}$ onto $L$.

We have thus proved the following

Theorem 6.2. The formal algebra $L$ of the standard Lie algebra sheaf $\mathscr{L}$ of type $\left(\mathrm{nt}, \mathfrak{g}^{0}, \ldots, \mathfrak{g}^{k}\right)$ may be identified with the formal algebra $\overline{\mathfrak{g}}$ associated with the prolongation $\mathfrak{g}$ of $\left(n 11, \mathfrak{g}^{0}, \ldots, \mathfrak{g}^{k}\right)$.

Let $G^{0}$ be a Lie subgroup of $G^{0}(111)$ and let $\left(P_{\#}, \omega\right)$ be the standard

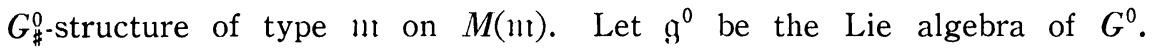
Then we notice that the standard Lie algebra sheaf $\mathscr{L}$ of type $\left(\mathfrak{m}, \mathfrak{g}^{0}\right)$ is nothing but the sheaf of all the local vector fields on $M(n)$ which induce infinitesimal automorphism of $\left(P_{\#}, \omega\right)$.

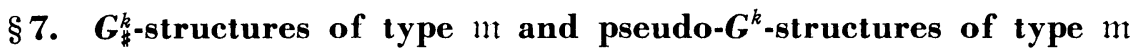

7.1. In this and the subsequent two sections, we shall consider a fixed fundamental graded algebra $m=\sum_{p<0} \mathfrak{g}^{p}$ of $\ell$-th kind over $\boldsymbol{R}$ and a 
fixed Lie subgroup $G^{0}$ of $G^{0}(111)$. We shall denote by $\mathfrak{g}^{0}$ the Lie algebra of $G^{0}$ and by $\mathfrak{g}=\sum_{p \in \boldsymbol{Z}} \mathfrak{g}^{p}$ the prolongation of $\left(\mathfrak{m}, \mathfrak{g}^{0}\right)$.

First of all, we shall define four series of Lie groups $H^{k}, N^{k}, G_{\sharp}^{k}$ and $G^{k}(k>0)$ together with operators $\partial: \mathfrak{s}^{k} \rightarrow \mathfrak{S}^{(k-1)}(k>0)$, which will play important roles for our later arguments.

We set $m^{k}=\sum_{p \leqq k} \mathfrak{g}^{p}$. Let $k$ be any integer $\geqq 0$. We set $\mathfrak{b}_{k}^{p}=\sum_{r=p}^{k} \mathfrak{g}^{r}$ $(p<0)$. Then we have $\mathfrak{s}_{k}^{p}=\mathfrak{m}^{k}(p \leqq-\mu)$ and

$$
\mathrm{m}^{k}=\boldsymbol{\delta}_{k}^{-\mu} \risingdotseq \cdots \mathrm{d}_{k}^{-1} \supsetneq \mathrm{g}^{k} .
$$

For each $p<k$, we define a $g^{p}$-valued linear form $\varepsilon_{k}^{p}$ on $\searrow_{k}^{p-k}$ by $\varepsilon_{k}^{p}(X)$ $=X^{p}$ for any $X \in \mathfrak{D}_{k}^{p-k}$. It is clear that $\varepsilon_{k}^{p}(p<-\mu)$ vanish and that $\varepsilon_{k}^{p}(p-k \leqq-\mu)$ are defined on the whole $m^{k}$.

The groups $H^{k}$ and $N^{k}(k>0)$. First we define $H^{k}$ to be the subgroup of $G L\left(n^{k-1}\right)$ which consists of all the elements a satisfying the following conditions:
i) $\quad a \mathfrak{D}_{k-1}^{p}=\mathfrak{D}_{k-1}^{p} \quad(p<0)$
ii) $a X^{k-1}=X^{k-1}$ for any $X^{k-1} \in \mathfrak{g}^{k-1}$,
iii) $\quad a^{*} \varepsilon_{k}^{p-1}=\varepsilon_{k-1}^{p} \quad(p \leqq k-2)$.

Let $a \in G L\left(\mathrm{~m}^{k-1}\right)$. Then it is easy to see that $a \in H^{k}$ if and only if

$$
\left(a \cdot X^{p}\right)^{r}=\delta_{p, r} X^{p}
$$

for any $X \in m^{k-1}$ and for any pair $(p, r)$ such that $r-k+1 \leqq p \leqq-2$ or $-1 \leqq p \leqq k-2, r \leqq k-2$ or $p=k-1, r \leqq k-1$, where $\left(\delta_{p, r}\right)$ denotes the Kronecker's symbol. We now define $N^{k}$ to be the subgroup of $G L\left(m^{k-1}\right)$ which consists of all the elements a satisfying (7.1) for any $X \in \mathrm{m}^{k-1}$ and for any pair $(p, r)$ such that $r-k \leqq p \leqq-2$ or $-1 \leqq p$ $\leqq k-1, r \leqq-1$. $\quad N^{k}$ is a normal subgroup of $H^{k}$.

The group $H^{k}$ consists of all the matrices $a$ of the form: 


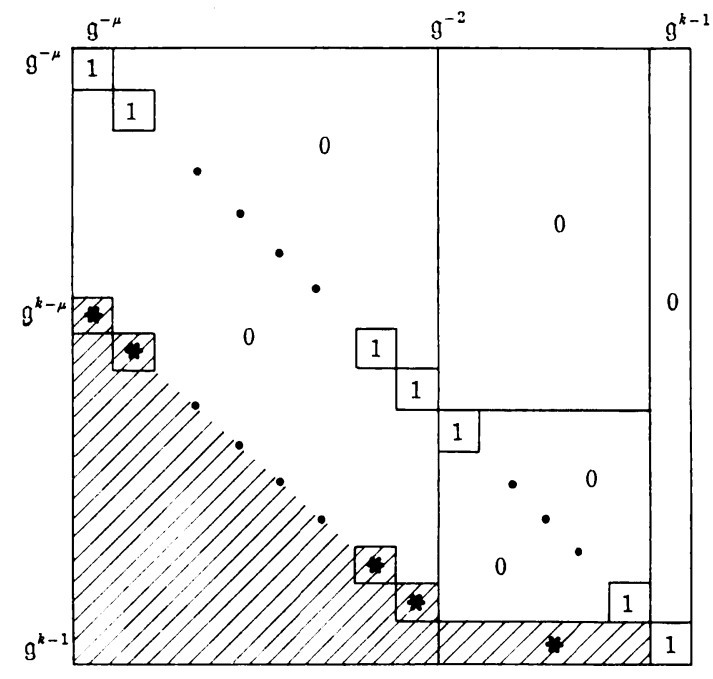

The subgroup $N^{k}$ of $H^{k}$ consists of all the matrices $a$ with $*$ replaced by 0 .

Let us observe the Lie algebra of $H^{k}$ and of $N^{k}$. We set as follows: $\mathfrak{g}^{k p}=\operatorname{Hom}\left(\mathfrak{g}^{p-k}, \mathfrak{g}^{p}\right) \quad(p<k-1), \mathfrak{g}^{k, k-1}=\operatorname{Hom}\left(\mathfrak{D}_{k-2}^{-1}, \mathfrak{g}^{k-1}\right), \quad \mathfrak{l}^{k p}$ $=\sum_{r<p-k} \operatorname{Hom}\left(\mathfrak{g}^{r}, \mathfrak{g}^{p}\right)(p<k), \mathfrak{s}^{k}=\sum_{p<k} \mathfrak{g}^{k p}, \mathfrak{l}^{k}=\sum_{p<k} \mathfrak{u}^{k p}$ and $\mathfrak{h}^{k}=\mathfrak{g}^{k}+\mathfrak{t}^{k}$. We have $\mathrm{m}^{k-1}=\sum_{p<k} \mathfrak{g}^{p}=\left(\sum_{p \leqq-2} \mathfrak{g}^{p}\right)+\mathfrak{D}_{k-2}^{-1}+\mathfrak{g}^{k-1}$, and we may identify $\mathfrak{h}^{k}$ with a subspace of $\left.\mathfrak{g l}^{(} \mathrm{m}^{k-1}\right)$. Then we see that $\mathfrak{h}^{k}\left(\right.$ resp. $\left.\mathfrak{u}^{k}\right)$ is the Lie algebra of $H^{k}$ (resp. $\left.N^{k}\right)$. Now define a mapping $t^{k}$ of $\mathfrak{h}^{k}$ to $G L\left(\mathrm{~m}^{k-1}\right)$ by $t^{k}(u) Y=Y+u(Y)$ for any $u \in \mathfrak{h}^{k}$ and $Y \in m^{k-1}$. It is clear that $t^{k}$ gives an injective mapping of $\mathfrak{h}^{k}\left(\right.$ resp. $\left.\mathfrak{u}^{k}\right)$ onto $H^{k}\left(\right.$ resp. $\left.N^{k}\right)$. We have $H^{k}=t^{k}\left(\mathfrak{g}^{k}\right) N^{k}$ and $t^{k}(u) t^{k}\left(u^{\prime}\right) \equiv t^{k}\left(u+u^{\prime}\right)\left(\bmod N^{k}\right)$ for any $u, u^{\prime} \in \mathfrak{g}^{k}$. If $k \geqq \mu$, then we have $t^{k}(u) t^{k}\left(u^{\prime}\right)=t^{k}\left(u+u^{\prime}\right)$ for any $u, u^{\prime} \in \mathfrak{S}^{k}$.

The operators $\partial: \mathfrak{g}^{k} \rightarrow \mathfrak{C}^{(k-1)}(k>0)$. We set $\mathfrak{C}_{k}^{p}=\operatorname{Hom}\left(\mathfrak{b}_{k-1}^{-1} \wedge \mathfrak{g}^{p-k}\right.$, $\left.\mathfrak{g}^{p}\right)(p<k-1), \mathfrak{S}_{k}^{k-1}=\operatorname{Hom}\left(\mathfrak{D}_{k-1}^{-1} \wedge \mathfrak{D}_{k-1}^{-1}, \mathfrak{g}^{k-1}\right)$ and $\mathfrak{S}^{(k)}=\sum_{p<k} \mathfrak{S}_{k}^{p}$. For each $u \in \mathfrak{g}^{k}$ and $p \leqq k-2$, define an element $(\partial u)^{p}$ of $\mathfrak{S}_{k-1}^{p}$ by

$$
\begin{aligned}
& (\partial u)^{p}(X \wedge Z)=[u(X), Z]-\left[u(Z), X^{-1}\right]-u\left(\left[X^{-1}, Z\right]\right)(p<k-2), \\
& (\partial u)^{p}(X \wedge Y)=\left[u(X), Y^{-1}\right]-\left[u(Y), X^{-1}\right]-u\left(\left[X^{-1}, Y^{-1}\right]\right)(p=k-2)
\end{aligned}
$$

for any $u \in \mathfrak{S}^{k}, X, Y \in \mathfrak{D}_{k-2}^{-1}$ and $Z \in \mathfrak{g}^{p-k+1}$. Thus we get a linear map- 
ping $\quad \partial: \mathfrak{s}^{k} \ni u \rightarrow \partial u=\sum_{p \leqq k-2}(\partial u)^{p} \in \mathfrak{C}^{(k-1)}$. We have $\mathfrak{g}^{k} \subset q^{k}=\sum_{r<0} \operatorname{Hom}\left(\mathfrak{g}^{r}\right.$, $\left.\mathfrak{g}^{r+k}\right) \subset \mathfrak{g}^{k}$, and we see that the kernel of $\partial: \mathfrak{g}^{k} \rightarrow \mathfrak{C}^{(k-1)}$ is equal to $\mathfrak{g}^{k}$.

The groups $G_{\sharp}^{k}$ and $G^{k}(k>0)$. First we define $G_{\sharp}^{k}$ to be the subgroup $t^{k}\left(\mathfrak{g}^{k}+\mathfrak{l t}^{k}\right)=t^{k}\left(\mathfrak{g}^{k}\right) \cdot N^{k}$ of $H^{k}$. Let $\mathfrak{g}_{\sharp}^{k}$ denote the Lie algebra $G_{\sharp}^{k}$. Then we have $\mathfrak{g}_{\sharp}^{k}=\mathfrak{g}^{k}+\mathfrak{l}^{k}$. Now we define $G^{k}$ to be the factor group $G_{\sharp}^{k} / N^{k}$. We see that $G^{k}$ is an abelian group and that its Lie algebra is given by $\mathrm{g}^{k}=\mathrm{q}_{\sharp}^{k} / \mathrm{ut}^{k}$. Note that the mapping $\mathrm{g}^{k} \ni X^{k} \rightarrow t^{k}\left(X^{k}\right) \in G_{\sharp}^{k}$ induces the exponential mapping exp of $\mathfrak{g}^{k}$ to $G^{k}$. Moreover, note that if $k \geqq \mu$, then $G^{k}$ may be identified with the subgroup $t^{k}\left(\mathfrak{g}^{k}\right)$ of $G_{\sharp}^{k}$.

Finally, let us observe the Lie group $G_{\#}^{0}=G^{0} \cdot N^{0}$. We denote by $\mathfrak{g}_{\sharp}^{0}\left(\right.$ resp. $\left.\mathfrak{n}^{0}\right)$ the Lie algebra of $G_{\sharp}^{0}\left(\right.$ resp. $\left.N^{0}\right)$. Then we have $\mathfrak{g}_{\sharp}^{0}=\mathfrak{g}^{0}$ $+\mathfrak{l}^{0}$, and we see that $\mathfrak{u}^{0}$, being a subalgebra of $\mathfrak{g l}(\mathfrak{l})$, may be expressed as $\sum_{p<0} \mathfrak{u}^{0 p}$, where $\mathfrak{u}^{0 p}=\sum_{r<p} \operatorname{Hom}\left(\mathfrak{g}^{r}, \mathfrak{g}^{p}\right)$. We denote by $t^{0}$ the injective mapping of $u^{0}$ onto $N^{0}$ defined by $t^{0}(u) Y=Y+u(Y)$ for all $u \in \mathfrak{n}^{0}$ and $Y \in \mathrm{m}$.

7.2. Let $k$ be any integer $\geqq 0$. For each $p \leqq k-2$, we denote by $I(k, p)$ the subset of $\boldsymbol{Z} \times \boldsymbol{Z}$ consisting of all the pairs $(r, s)$ such that $-1 \leqq r, s \leqq p$ or $p-k<r, s<k, r+s<p$. If $(r, s) \in I(k, p)$, we have $r, s \leqq k-2$. We have $I(0, p)=I(p)(p \leqq-2)$.

Since $G_{\#}^{k}$ is a Lie subgroup of $G L\left(m^{k-1}\right)$, we have the notion of $G_{\sharp}^{k}$-structures. We set $m^{k-1}=\operatorname{dim} m^{k-1}$. Let $M^{k-1}$ be a manifold of dimension $m^{k-1}$, and let $\left(P_{\#}^{k}, \omega^{(k)}\right)$ be a $G_{\sharp}^{k}$-structure on $M^{k-1}$. $\omega^{(k)}$ being an $\mathrm{m}^{k-1}$-valued 1 -form on $P_{\sharp}^{k}$, we denote by $\omega_{k}^{p}$ the $\mathfrak{g}^{p}$-component of $\omega^{(k)}$ in the decomposition: $m^{k-1}=\sum_{p<k} \mathfrak{g}^{p}$. The following definition generalizes Def. 4.1.

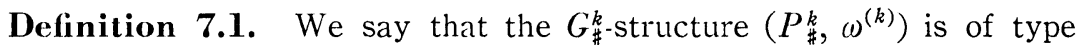
$m$ if it satisfies the following equalities

$$
\begin{aligned}
& \Omega_{k}^{p}=d \omega_{k}^{p}+\frac{1}{2} \sum_{r+s=p}\left[\omega_{k}^{r}, \omega_{k}^{s}\right] \equiv 0 \\
& \left\{\bmod \omega_{k}^{r}(r \leqq p-k) ; \omega_{k}^{r} \wedge \omega_{k}^{s}((r, s) \in I(k, p))\right\} \quad(p \leqq k-2)
\end{aligned}
$$

7.3. Definition 7.2. Let $M^{k-1}$ be a manifold of dimension $m^{k-1}$ 
$(k \geq 0)$, and let $P^{k}$ be a principal fiber bundle over the base space $M^{k-1}$ with structure group $G^{k}$. Let $D^{(k)}=\left(D_{k}^{p}\right)_{p<0}$ be a family of differential systems on $P^{k}$ and let $\theta^{(k)}=\left(\theta_{k}^{p}\right)_{p<k}$ be a family of forms such that $\theta_{k}^{p}$ is a $\mathrm{g}^{p}$-valued 1 -form ${ }^{3)}$ on $\left(P^{k}, D_{k}^{p-k}\right)$. Assume the following conditions for the system $\left(P^{k}, D^{(k)}, \theta^{(k)}\right)$ :

1) $\operatorname{dim} D_{k}^{p}=\operatorname{dim} \mathbb{D}_{k}^{p}(p<0)$, and

$$
\cdots \supset D_{k}^{p} \supset \cdots \supset D_{k}^{-1} \supset D_{k}^{0}
$$

where $D_{k}^{0}$ denotes the differential system on $P^{k}$ consisting of all the vertical vectors on $P^{k}$.

2) For each $p<0, D_{k}^{p}$ is invariant under the action of $G^{k}$ on $P^{k}$.

3) For each $p<0, D_{k}^{p}$ is defined by the equations $\theta_{k}^{r}=0 \quad(r<p)$, and $D_{k}^{0}$ is defined by the equations $\theta_{k}^{r}=0(r<k)$.

4) Let $a \in G^{k}$, and express it as $\exp X^{k}\left(X^{k} \in \mathfrak{g}^{k}\right)$ when $k>0$.

If $k=0$,

$$
R(a)^{*}=a^{-1} \theta_{0}^{p} \quad(p<0)
$$

and if $k>0$,

$$
R(a)^{*} \theta_{k}^{p}=\theta_{k}^{p}-\left[X^{k}, \theta_{k}^{p-k} \mid D_{k}^{p-k}\right](p<k),
$$

where $R(a)$ denotes the right translation of $P^{k}$ induced by $a$.

Under these conditions, we say that the system $\left(P^{k}, D^{(k)}, \theta^{(k)}\right)$ is a pseudo- $G^{k}$-structure on $M^{k-1}$.

Remark 1. Let $\left(P^{k}, D^{(k)}, \theta^{(k)}\right)$ be a pseudo- $G^{k}$-structure. Then we have: i) $\theta_{k}^{p}=0(p<-\mu)$, ii) $D_{k}^{p}=T\left(P^{k}\right)(p \leqq-\mu)$, and hence $\theta_{k}^{p}$ $(p-k \leqq-\mu)$ are usual forms defined on $P^{k}$.

Definition 7.3. Let $\left(P^{k}, D^{(k)}, \theta^{(k)}\right)\left\{\right.$ resp. $\left.\left(P^{\prime k}, D^{\prime(k)}, \theta^{\prime(k)}\right)\right\}$ be a

3) Let $D$ be a differential system on a manifold $M$. As for the definition of differential forms defined on $(M, D)$, see $[13]$, $§ 1$. 
pseudo- $G^{k}$-structure on a manifold $M^{k-1}$ (resp. $M^{\prime k-1}$ ). We say that a bundle isomorphism $\varphi$ of $P^{k}$ onto $P^{\prime k}$ is an isomorphism of $\left(P^{k}, D^{(k)}\right.$, $\left.\theta^{(k)}\right)$ onto $\left(P^{\prime k}, D^{\prime(k)}, \theta^{(k)}\right)$ if we have the equalities:

$$
\begin{aligned}
& \varphi^{*} D_{k}^{\prime p}=D_{k}^{p} \quad(p<0), \\
& \varphi^{*} \theta_{k}^{\prime p}=\theta_{k}^{p} \quad(p<k) .
\end{aligned}
$$

Definition 7.4. Let $\left(P^{k}, D^{(k)}, \theta^{(k)}\right)$ be a pseudo- $G^{k}$-structure on a manifold $M^{k-1}$. We say that an $\mathrm{m}^{k-1}$-valued 1 -form $\xi^{k-1}$ on $P^{k}$ is compatible with $\theta^{(k)}$ if we have the equalities:

$$
\xi_{k}^{p} \mid D_{k}^{p-k}=\theta_{k}^{p} \quad(p<k)
$$

where $\xi_{k}^{p}$ denotes the $\mathrm{g}^{p}$-component of $\xi^{(k)}$ in the decomposition $\mathrm{m}^{k-1}$ $=\sum_{p<k} \mathfrak{g}^{p}$.

Remark 2. Let $\xi^{(k)}$ be a 1 -form compatible with $\theta^{(k)}$. By condition 3) in Def. 7.2, we see that $D_{k}^{p}$ is defined by the equations $\xi_{k}^{r}$ $=0(r<p)$. Let $\eta^{(k)}$ be a second 1 -form compatible with $\theta^{(k)}$. Then it follows that

$$
\eta_{k}^{p} \equiv \xi_{k}^{p}\left\{\bmod \xi_{k}^{r}(r<p)\right\} \quad(p<k) .
$$

Definition 7.5. We say that a pseudo- $G^{k}$-structure $\left(P^{k}, D^{(k)}, \theta^{(k)}\right)$ on a manifold $M^{k-1}$ is of type $n$ if there is an $\mathrm{m}^{k-1}$-valued 1 -form $\xi^{(k)}$ on $P^{k}$ which is compatible with $\theta^{(k)}$ and which satisfies the equalities :

$$
\begin{aligned}
\Xi_{k}^{p}= & d \xi_{k}^{p}+\frac{1}{2} \sum_{r+s=p}\left[\xi_{k}^{r}, \xi_{k}^{s}\right] \equiv 0 \\
& \left\{\bmod \xi_{k}^{r}(r \leqq p-k) ; \xi_{k}^{r} \wedge \xi_{k}^{s}((r, s) \in I(k, p))\right\} \quad(p \leqq k-2) .
\end{aligned}
$$

Remark 3. Let $\left(P^{k}, D^{(k)}, \theta^{(k)}\right)$ be a pseudo- $G^{k}$-structure of type $\mathfrak{m}$. Then we see from Remark 2 that every 1 -form $\eta^{(k)}$ compatible with $\theta^{(k)}$ satisfies the equalities in Def. 7.5. 


\section{§8. The prolongation theorems}

8.1. Let $\left(P_{\sharp}^{k}, \omega^{(k)}\right)$ be a $G_{\sharp}^{k}$-structure on a manifold $M^{k-1}$. For each $p<0$, we denote by $D_{\# k}^{p}$ the differential system on $P \sharp$ defined by the equations $\omega_{k}^{r}=0(r<p)$. Then we have

$$
\cdots \supset D_{\# k}^{p} \supset \cdots \supset D_{\# k}^{-1} \supset D_{\# k}^{0},
$$

where $D_{\# k}^{0}$ denotes the differential system on $P_{\sharp}^{k}$ consisting of all the vertical vectors in $P_{\sharp}^{k}$. We have $\operatorname{dim} D_{\# k}^{p}=\operatorname{dim} \mathfrak{D}_{k}^{p}+\operatorname{dim} \mathfrak{n}^{k}(p<0)$.

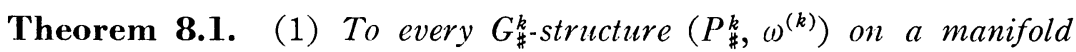
$M^{k-1}(k \geqq 0)$, there is associated, in a natural way, a pseudo-G ${ }^{k}$-structure $\left(P^{k}, D^{(k)}, \theta^{(k)}\right)$ on $M^{k-1}$ having the following properties:

1) The principal fiber bundle $P^{k}$ is equal to the quotient $P_{\sharp}^{k} / N^{k}$ of $P_{\#}^{k}$ by the normal subgroup $N^{k}$ of $G^{k}$ which is a principal fiber bundle over the base space $M^{k-1}$ with structure group $G^{k}=G_{\sharp}^{k} / N^{k}$.

2) Denote by $\beta$ the projection of $P_{\sharp}^{k}$ onto $P^{k}=P_{\sharp}^{k} / N^{k}$. Then we have

$$
\begin{aligned}
& \beta^{*} D_{k}^{p}=D_{\# k}^{p} \quad(p<0), \\
& \beta^{*} \theta_{k}^{p}=\omega_{k}^{p} \mid D_{\# k}^{p-k} \quad(p<k) .
\end{aligned}
$$

(2) If $\left(P_{\sharp}^{k}, w^{(k)}\right)$ is of type $\mathrm{m}$, so is $\left(P^{k}, D^{(k)}, \theta^{(k)}\right)$.

(3) The assignment $\left(P_{\#}^{k}, \omega^{(k)}\right) \rightarrow\left(P^{k}, D^{(k)}, \theta^{(k)}\right)$ is compatible with the respective isomorphisms. Namely if $\left(P_{\#}^{k}, \omega^{(k)}\right) \rightarrow\left(P^{k}, D^{(k)}, \theta^{(k)}\right)$ and $\left(P_{\sharp}^{\prime(k)}, \omega^{\prime(k)}\right) \rightarrow\left(P^{\prime k}, D^{\prime(k)}, \theta^{\prime(k)}\right)$, then we have:

i) Every isomorphism $\varphi_{\#}$ of $\left(P_{\#, \omega^{k}}^{(k)}\right)$ onto $\left(P_{\#}^{\prime k}, \omega^{(k)}\right)$ induces a unique isomorphism $\varphi$ of $\left(P^{k}, D^{(k)}, \theta^{(k)}\right)$ onto $\left(P^{\prime(k)}, D^{\prime(k)}, \theta^{\prime(k)}\right)$.

ii) Every isomorphism $\varphi$ of $\left(P^{k}, D^{(k)}, \theta^{(k)}\right)$ onto $\left(P^{\prime k}, D^{\prime(k)}, \theta^{\prime(k)}\right)$ is induced by a unique isomorphism $\varphi_{\#}$ of $\left(P_{\#}^{k}, \omega^{(k)}\right)$ onto $\left(P_{\#}^{\prime k}, \omega^{\prime(k)}\right)$.

Theorem 8.2. Assume that $G^{0}$ is connected and, for each $k \geqq 1$, choose a complementary subspace $\mathfrak{F}_{\sharp}^{(k-1)}$ of $\partial \mathfrak{S}^{k}$ in $\mathfrak{(}^{(k-1)}$.

(1) To every pseudo-G $G^{k-1}$-structure $\left(P^{k-1}, D^{(k-1)}, \theta^{(k-1)}\right)$ of type $\mathrm{m}$ 
on a manifold $M^{k-2}(k \geqq 1)$, there is associated, in a canonical manner,

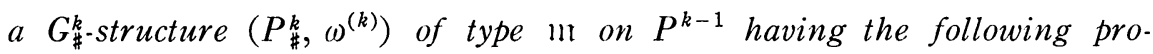
perties: Denote by $\alpha$ the projection of $P_{\#}^{k}$ onto $P^{k-1}$. Then we have

$$
\begin{aligned}
& \alpha^{*} D_{k-1}^{p}=D_{\sharp k}^{p} \quad(p<0), \\
& \alpha^{*} \theta_{k-1}^{p}=\omega_{k}^{p} \mid D_{\# k}^{p-k+1} \quad(p \leqq k-2) .
\end{aligned}
$$

(2) The assignment $\left(P^{k-1}, D^{(k-1)}, \theta^{(k-1)}\right) \rightarrow\left(P_{\sharp}^{k}, \omega^{(k)}\right)$ is compatible with the respective isomorphisms. Namely if $\left(P^{k-1}, D^{(k-1)}, \theta^{(k-1)}\right) \rightarrow\left(P_{k}^{\sharp}, \omega^{(k)}\right)$ and $\left(P^{\prime k-1}, D^{\prime(k-1)}, \theta^{(k-1)}\right) \rightarrow\left(P_{\#}^{\prime k}, \omega^{\prime(k)}\right)$, then we have:

1) Every isomorphism $\varphi$ of $\left(P^{k-1}, D^{(k-1)}, \theta^{(k-1)}\right)$ onto $\left(P^{\prime k-1}\right.$, $\left.D^{(k-1)}, \theta^{(k-1)}\right)$ is induced by a unique isomorphism $\varphi_{\#}$ of $\left(P_{\#}^{k}, \omega^{(k)}\right)$ onto $\left(P_{\#}^{\prime k}, \omega^{\prime(k)}\right)$.

2) Every isomorphism $\varphi_{\#}$ of $\left(P^{k}, \omega^{(k)}\right)$ onto $\left(P_{\#}^{\prime k}, \omega^{(k)}\right)$ induces a unique isomorphism $\varphi$ of $\left(P^{k-1}, D^{(k-1)}, \theta^{(k-1)}\right)$ onto $\left(P^{\prime k-1}, D^{(k-1)}, \theta^{(k-1)}\right)$.

Th. 8.1 and Th. 8.2 will be proved in the next section.

Theorem 8.3. Assume that $G^{0}$ is connected and, for each $k \geqq 1$, choose a complementary subspace $\mathbb{E}_{\sharp}^{(k-1)}$ of $\partial \mathfrak{s}^{k}$ in $\mathbb{(}^{(k-1)}$.

(1) To every pseudo-G $G^{0}$-structure $\left(P^{0}, D^{(0)}, \theta^{(0)}\right)$ of type $\mathrm{m}$ on a manifold $M^{-1}$, there is associated, in a canonical manner, a sequence $(P):\left(P^{0}, D^{(0)} \theta^{(0)}\right) \leftarrow \cdots \leftarrow\left(P^{k-1}, D^{(k-1)}, \theta^{(k-1}\right) \stackrel{\boldsymbol{\sigma}^{k}}{\longrightarrow}\left(P^{k}, D^{(k)}, \theta^{(k)}\right) \leftarrow \cdots$ as follows: 1) For each $k \geqq 1,\left(P^{k}, D^{(k)}, \theta^{(k)}\right)$ is a pseudo-G $G^{k}$-structure of type $\mathrm{m}$ on $P^{k-1}$, and $\sigma^{k}$ is the projection of $P^{k}$ onto $P^{k-1}$.

2) For each $k \geqq 1$, we have

$$
\begin{aligned}
& \sigma^{k *} D_{k-1}^{p}=D_{k}^{p} \quad(p<0), \\
& \sigma^{k *} \theta_{k-1}^{p}=\theta_{k}^{p} \mid D_{k}^{p-k+1} \quad(p \leqq k-2) .
\end{aligned}
$$

(2) The assignment $\left(P^{0}, D^{(0)}, \theta^{(0)}\right) \rightarrow(P)$ is compatible with the various isomorphisms.

Proof. This follows immediately from Th. 8.1 and Th. 8.2. In- 
deed, let $\left(P^{k-1}, D^{(k-1)}, \theta^{(k-1)}\right)$ be a pseudo- $G^{k-1}$-structure of type $m$ on a manifold $M^{k-2}$. By Th. 8.2, there is attached to $\left(P^{k-1}, D^{(k-1)}, \theta^{(k-1)}\right)$ a $G_{\sharp}^{k}$-structure $\left(P_{\#}^{k}, \omega^{(k)}\right)$ of type $\|$ on $P^{k-1}$. By Th. 8.1, $\left(P_{\#}^{k}, \omega^{(k)}\right)$ gives rise to a pseudo- $\mathrm{G}^{k}$-structure $\left(P^{k}, \theta^{(k)}\right)$ of type $\|$ on $P^{k-1}$. Let $\alpha^{k}$ (resp. $\beta^{k}$, resp. $\left.\sigma^{k}\right)$ denote the projection of $P_{\#}^{k}\left(\right.$ resp. $P_{\sharp}^{k}$, resp. $\left.P^{k}\right)$ onto $P^{k-1}$ (resp. $P^{k}$, resp. $P^{k-1}$ ). Then we have $\sigma^{k} \circ \beta^{k}=\alpha^{k}$. Therefore, for any $p<0$, we get $\beta^{k *}\left(\pi^{k *} D_{k-1}^{p}\right)=\alpha^{k *} D_{k-1}^{p}=D_{\# k}^{p}=\beta^{k *} D_{k}^{p}$, whence $\varpi^{k *} D_{k-1}^{p}=D_{k}^{p}$. Moreover, for any $p \leqq k-2$, we get $\beta^{k *} \varpi^{k *} \theta_{k-1}^{p}$ $=\alpha^{k *} \theta_{k-1}^{p}=\omega_{k}^{p}\left|D_{\# k}^{p-k+1}=\left(\beta^{k *} \theta_{k}^{p}\right)\right| \beta^{k *} D_{k}^{p-k+1}=\beta^{k *}\left(\theta_{k}^{p} \mid D_{k}^{p-k+1}\right)$, whence $\sigma^{k *} \theta_{k-1}^{p}=\theta_{k}^{p} \mid D_{k}^{p-k+1}$. The iterative applications of the assignments $\left(P^{k-1}, D^{(k-1)}, \theta^{(k-1)}\right) \rightarrow\left(P^{k}, D^{(k)}, \theta^{(k)}\right)$ yield the required sequence $(P)$.

The sequence $(P)$ is called the prolongation of the pseudo- $G^{0}$. structure $\left(P^{0}, D^{(0)}, \theta^{(0)}\right)$.

Remark 1. It is easy to see that the assignment $\left(P_{\#}^{k}, \omega^{(k)}\right) \rightarrow$ $\left(P^{k}, D^{(k)}, \theta^{(k)}\right)$ in Th. 8.1 has a local property: More precisely, let $U$ be an onen set of $M^{k-1}$. Then the pseudo- $G^{k}$-structure corresponding to the restriction of $\left(P_{\sharp}^{k}, \omega^{(k)}\right)$ to $U$ is just the restriction of $\left(P^{k}, D^{(k)}\right.$, $\left.\theta^{(k)}\right)$ to $U$. From the proof of Th. 8.2, we shall find that the assignment $\left(P^{k-1}, D^{(k-1)}, \theta^{(k-1)}\right) \rightarrow\left(P_{\#}^{k}, \omega^{(k)}\right)$ in Th. 8.2 also has a local property: More precisely, let $U$ be an open set of $M^{k-2}$. Then the $G_{\#}^{k}$-structure corresponding to the restriction of $\left(P^{k-1}, D^{(k-1)}, \theta^{(k-1)}\right)$ to $U$ is just the restriction of $\left(P_{\#,}^{k}, \omega^{(k)}\right)$ to $\sigma^{-1}(U)$, $\sigma$ being the projection of $P^{k-1}$ onto $M^{k-2}$. It follows that the assignment $\left(P^{0}, D^{(0)}, \theta^{(0)}\right) \rightarrow(P)$ in Th. 8.3 has a local property in a suitable sense.

8.2. Theorem 8.4. Assume that the pair $\left(\mathrm{n}, \mathrm{g}^{0}\right)$ is of finite type, and let $\left(P_{\sharp},(0)\right.$ be a $G_{\sharp}^{0}$-structure of type $\mathrm{nt}$ on a connected manifiold $M$. Then the Lie algebra of all the infinitesimal automorphisms of $\left(P_{\#}, \omega\right)$ is finite dimensional and of dimension $\leqq \operatorname{dim} \mathfrak{g}$.

Corollary. Let $\mathrm{m}$ be a fundamental graded algebra of finite type, and let $D$ be a regular differential system of type $\mathrm{nt}$ on a connected manifold $M$ of dimension $m=\operatorname{dim} \mathrm{m}$. Then the Lie algebra of all the infinitesimal automorphisms of $(M, D)$ is finite dimensional and of 
dimension $\leqq \operatorname{dim} \mathfrak{g}(\mathrm{m})$.

Proof of Th. 8.4. Let $\left(P^{0}, D^{(0)}, \theta^{(0)}\right)$ be the pseudo- $G^{0}$-structure

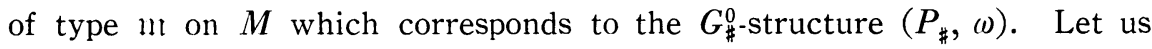
consider the sequence $(P)$ in Th. 8.3. Let $l$ denote the largest $p \geqq-1$ such that $\mathrm{g}^{p} \neq\{0\}$. Setting $k=l+\iota$, then we have $P^{l}=P^{k}$, where we put $P^{-1}=M$. It is clear that the forms $\theta_{k}^{p}(p<-\mu$ or $p>l)$ vanish and that the forms $\theta_{k}^{p}(-\mu \leqq p \leqq l)$ are usual forms defined on $P^{l}$. We have $\operatorname{dim} P^{l}=\sum_{p=-\mu}^{l} \operatorname{dim} \mathrm{g}^{p}=\operatorname{dim} \mathrm{g}$, and we know that the forms $\theta_{k}^{p}(-\mu \leqq$ $p \leqq l)$ define an absolute parallelism on $P^{l}$, i.e., the mapping $T_{z}\left(P^{l}\right)$ $\ni X \stackrel{l}{\rightarrow} \sum_{p=-\mu} \theta_{k}^{p}(X) \in \mathfrak{g}$ gives a linear isomorphism at each $z \in P^{l}$. Therefore, Th. 8.4 follows from Th. 8.1 (applied for $k=0$ ) and Th. 8.3.

8.3. We shall introduce the notion of the structure functions of a pseudo- $G^{0}$-structure of type 11 .

For any integer $p \geqq-\mu$, we denote by $A(p)$ (resp. $B(p)$ ) the subset $\boldsymbol{Z} \times \boldsymbol{Z}$ which consists of all the elements $(r, s)$ such that $r+s$ $=p,-\mu \leqq r, s$ (resp. $-1 \leqq r, s \leqq p$ or $r+s<p,-\mu \leqq r, s<p+\mu)$. Let $\left(P^{0}, D^{(0)}, \theta^{(0)}\right)$ be a pseudo- $G^{0}$-structure of type $\|$ on a manifold $M$ and let us consider its prolongation $(P)$.

Lemma 8.1. Let $(k, p)$ be any pair of integers with $\mu \leqq k,-\mu$ $\leqq p \leqq k-2 \mu$, and consider the pseudo- $G^{k}$-structure, $\left(P^{k}, D^{(k)}, \theta^{(k)}\right)$, of type $n$ in on $P^{k-1}$.

(1) The forms $\theta_{k}^{p}$ and $\theta_{s}^{r}, \theta_{k}^{s}((r, s) \in A(p) \cup B(p))$ are usual forms defined on $P^{k}$.

(2) $\Theta_{k}^{p}=d \theta_{k}^{p}+\frac{1}{2} \sum_{(r, s) \in A(p)}\left[\theta_{k}^{r}, \theta_{k}^{s}\right] \equiv 0 \quad\left\{\bmod \theta_{k}^{r} \wedge \theta_{k}^{s}((r, s) \in B(p))\right\}$.

Proof. (1) is clear. Let us prove (2). Let $\xi^{(k)}$ be an $\mathfrak{m}^{k-1}$-valued 1 -form on $P^{k}$ compatible with $\theta^{(k)}$. Then we have $\xi_{k}^{p}=\theta_{k}^{p}$ and $\xi_{k}^{r}$ $=\theta_{k}^{r}, \xi_{k}^{s}=\theta_{k}^{s}$ for any $(r, s) \in A(p) \cup B(p)$. Furthermore we find that $B(p)$ consists of all $(r, s) \in I(k, p)$ with $r, s \geqq-\mu$. Therefore (2) follows from the equality in Def. 7.5. 
We put $\Re^{p}=\sum_{(r, s) \in B(p)} \operatorname{Hom}\left(\mathfrak{g}^{r} \wedge \mathfrak{g}^{s}, \mathfrak{g}^{p}\right)(p \geqq-\mu)$. Let $k$ be any integer $\geqq \mu$. By Lemma 8.1, we know that, for each $p(-\mu \leqq p \leqq k-2 \mu)$, there is a unique $\Re^{p}$-valued function $K_{k}^{p}$ on $P^{k}$ satisfying the following equality :

$$
\Theta_{k}^{p}+\frac{1}{2} \sum_{(r, s) \in B(p)} K_{k}^{p}\left(\theta_{k}^{r} \wedge \theta_{k}^{s}\right)=0 .
$$

We shall say that the sum $K^{(k)}=\sum_{q=-\mu}^{k-2 \mu} K_{k}^{p}$ is the $k$-th structure function of $\left(P^{0}, D^{(0)}, \theta^{(0)}\right)$ and that the system of equations (8.1) for all $p(-\mu$ $\leqq p \leqq k-2 \mu)$ is the $k$-th structure equation of $\left(P^{0}, D^{(0)}, \theta^{(0)}\right)$.

Theorem 8.5. Suppose that the pseudo-G $G^{0}$-structure $\left(P^{0}, D^{(0)}, \theta^{(0)}\right)$

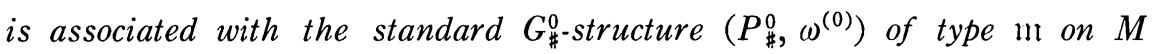
$=M(\mathrm{~m})$. Then the structure functions $K^{(k)}$ vanish for all. $k \geqq \mu$.

This theorem will follow from Lemma 8.2 which we shall explain from now on. Let $a=\sum_{p \leqq l} a^{p}$ be any element of $\mathfrak{g}$, where $a^{p} \in \mathfrak{g}^{p}$. We denote by $\sigma^{-1}(a)$ the vector field $X$ on $M(n t)$ defined by the equalities in Lemma 6.3. Then we see that the mapping $a \rightarrow \sigma^{-1}(a)$ is linear and that $\left[\sigma^{-1}(a), \sigma^{-1}(b)\right]=-\sigma^{-1}([a, b])$ (Lemma 6.4). Moreover we know that $\sigma^{-1}(a)$ induces an infinitesimal automorphism $\sigma_{\#}^{0}(a)$ of $\left(P_{\#}^{0}\right.$, $\left.\omega^{(0)}\right)$ (See 6.4). Therefore it induces, for each $k \geqq 0$, an infinitesimal automorphism $\sigma^{k}(a)$ of $\left(P^{k}, D^{(k)}, \theta^{(k)}\right)$ (Th. 8.1, applied for $k=0$, and Th. 8.3).

Lemma 8.2. For each $k \geqq 0$, we have the following proposition $\left(S^{k}\right)$ : There are a point $e^{k} \in P^{k}$ and an $\mathrm{m}^{k-1}$-valued 1 -form $\xi^{(k)}$ on $P^{k}$, compatible with $\theta^{(k)}$, which satisfy the following conditions:

1) $\xi_{k}^{r}\left(\sigma^{k}\left(a^{p}\right)_{e^{k}}\right)=\delta_{r p} a^{p}$

for any $a^{p} \in \mathrm{g}^{p}(p<k)$ and any $r<k$.

2) $\sigma^{k}\left(a^{k}\right)_{e^{k}}=r\left(a^{k}\right)_{e^{k}}$

for any $a^{k} \in \mathrm{g}^{k}$, where $r\left(a^{k}\right)$ denotes the vertical vector field of the principal fiber bundle $P^{k}\left(P^{k-1}, G^{k}\right)$ induced by $a^{k}$. 
3) $\sigma^{k}\left(a^{p}\right)_{e^{k}}=0$

for any $a^{p} \in \mathfrak{g}^{p}(p>k)$.

This lemma will be proved in the next section.

Proof of Th. 8.5. We have $\left[\sigma^{k}(a), \sigma^{k}(b)\right]=-\sigma^{k}([a, b])$ and $L_{\sigma^{k}(a)} \theta_{k}^{p}=0$. It follows that $\Theta_{k}^{p}\left(\sigma^{k}(a) \wedge \sigma^{k}(b)\right)=-\theta_{k}^{p}\left(\sigma^{k}([a, b])\right)+\sum_{\left.(r, s) \in B^{i} p\right)}$ $\left[\theta_{k}^{r}\left(\sigma^{k}(a)\right), \theta_{k}^{s}\left(\sigma^{k}(b)\right)\right]$. Therefore we have $\Theta_{k}^{p}\left(\sigma^{k}(a)_{e^{k}} \wedge \sigma^{k}(b)_{e^{k}}\right)=0$ by Lemma 8.2. Since the mapping $a \rightarrow \sigma^{k}(a)_{e^{k}}$ maps $\mathfrak{g}$ onto $T_{e^{k}}\left(P^{k}\right)$ (Lemma 8.2), we have $\left(\Theta_{k}^{p}\right)_{e^{k}}=0$. Moreover it follows that $\Theta_{k}^{p}=0$ on a neighborhood of $e^{k}$, because $L_{\sigma^{k}(a)} \Theta_{k}^{p}=0$. Since $P^{k}$ and $\Theta_{k}^{p}$ are real analytic and since $P^{k}$ is connected, we get $\Theta_{k}^{p}=0$. Thus we have proved $K^{(k)}=0$.

A converse of Th. 8.5 is also true (at least) in the case where $\left(\mathrm{n}, \mathfrak{g}^{0}\right)$ is of finite type. Indeed, let $\left(P_{\sharp}, \omega\right)$ be a $G_{\sharp}^{0}$-structure of type $n$ and let $\left(P^{0}, D^{(0)}, \theta^{(0)}\right)$ be the corresponding pseudo- $G^{0}$-structure. Let $l$ be the largest $p \geqq-1$ such that $\mathrm{g}^{p} \neq\{0\}$ and put $k=l+2 \mu$. If the $k$-th structure function $K^{(k)}$ of $\left(P^{0}, D^{(0)}, \theta^{(0)}\right)$ vanishes, then the given

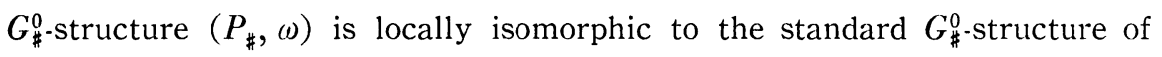
type ur. For the proof of this fact, we note that the family $\left(\theta_{k}^{p}\right)_{-\mu \leqq p \leqq l}$ gives an absolute parallelism on $P^{l}=P^{k}$ and that $\Theta_{k}^{p}=0(-\mu \leqq p \leqq l)$.

8.4. Remark 2. Let us define a decreasing sequence $\left(G_{*}^{k}\right)_{k>0}$ of Lie subgroups of $G_{\sharp}^{0}=G^{0} \cdot N^{0}$ as follows: Put $\mathfrak{q}_{-}^{p}=\sum_{r<0} \operatorname{Hom}\left(\mathfrak{g}^{r-p}, \mathfrak{g}^{r}\right)$ and $\mathfrak{q}_{+}^{p}=\sum_{0 \leq r<p} \operatorname{Hom}\left(\mathfrak{g}^{r-p}, \mathfrak{g}^{r}\right)(p>0)$. Then we have $\mathfrak{q}^{p}=\mathfrak{q}_{-}^{p}+\mathfrak{q}_{+}^{p}$, and we denote by $\lambda$ the projection of $q^{p}$ onto $q^{p}-$ with respect to this decomposition. Noting that $\mathfrak{g}^{p}$ is a subspace of $\mathfrak{q}^{p}$, we put $\mathfrak{n}_{*}^{k}=\underset{0<p \leqq k}{=} \lambda\left(\mathfrak{g}^{p}\right)+\sum_{k<p} \mathfrak{q}_{-}^{p}$. Then we find that $\mathfrak{u}_{*}^{k}$ is a subalgebra of $\mathfrak{n}^{0}=\sum_{p>0} q_{-}^{p}$ and that $\mathfrak{u}^{0} \supset \mathfrak{u}_{*}^{1} \supset \cdots \supset \mathfrak{u}_{*}^{\mu-1}$ $=\mathfrak{n}_{*}^{\mu}=\cdots$. Denote by $N_{*}^{k}$ the connected Lie subgroup of $N^{0}$ generated by the subalgebra $\mathfrak{n}_{*}^{k}$ of $\mathfrak{n}^{0}$, i.e., $N_{*}^{k}=t^{0}\left(\mathfrak{n}_{*}^{k}\right)$. Then the product $G_{*}^{k}$ $=G^{0} \cdot N_{*}^{k}$ is proved to be a (closed) subgroup of $G_{\#}^{0}$. (The proof uses the fact that every $a \in G^{0}$ is uniquely extended to an automorphism of the graded algebra g). We clearly have $G_{\#}^{0} \supset G_{*}^{1} \supset \cdots \supset G_{*}^{\mu-1}=G_{*}^{\mu}=\cdots$. It should be remarked that the Lie algebra of $G_{*}^{\mu-1}$ may be regarded as 
the linear isotropy algebra of the standard Lie algebra sheaf of type $\left(\mathrm{m}, \mathrm{g}^{0}\right)$.

Now we have proved "the first reduction theorem" which may roughly be stated as follows: Under a suitable condition $\left(C_{1}\right)$ on the pair $\left(\mathrm{m}, G^{0}\right)$, every $G_{\#}^{0}$-structure $\left(P_{\#},(1)\right.$ of type $\mathrm{m}$ on a manifold $M$ is reduced, in a canonical manner, to a $G_{*}^{1}$-structure $\left(P_{*}^{1}, \omega\right)$ on $M$ in such a way that the assignment $\left(P_{\#}, \omega\right) \rightarrow\left(P_{*}^{1}, \omega\right)$ is compatible with the respective isomorphisms.

Note that condition $\left(C_{1}\right)$ is satisfied if $G^{0}$ is connected and if the representation of $G^{0}$ on $\mathfrak{g}^{-1}$ is completely reducible. It is natural to expect that one can continue analogous reductions under further suitable conditions on the pair $\left(\mathrm{m}, G^{0}\right)$ to obtain a decreasing sequence of $G_{*}^{k}$ subbundles of $P_{\sharp}^{0}: P_{\#}^{0} \supset P_{*}^{1} \supset \cdots \supset P_{*}^{\mu-1}=P_{*}^{\mu}=\cdots$.

For example, consider the case where $\mu=3, \operatorname{dim} \mathfrak{g}^{-3}=2, \operatorname{dim} g^{-2}$ $=1$ and $\operatorname{dim} \mathfrak{g}^{-1}=2$ and where $G^{0}=G^{0}$ (int). (cf. 5.3, Example (2)). Then it can be shown that the pair $\left(n, G^{0}\right)$ satisfies condition $\left(C_{1}\right)$ and hence that every $G_{\sharp}^{0}$-structure $\left(P_{\#}, \omega\right)$ on $M$ is reduced to a $G_{*}^{1}$-structure $\left(P_{*}^{1}, \omega\right)$ on $M$ in the canonical manner. Moreover, we can show that the $G_{*}^{1}$-structure $\left(P_{*}^{1}, \omega\right)$ on $M$ is reduced to a $G_{*}^{2}$-structure $\left(P_{*}^{2}, \omega\right)$ on $M$ in a canonical manner. In his paper [1], p. 965-971, E. Cartan has really carried out such reductions in a "messy and complicated" manner. Finally, we mention that the Lie algebra $g_{\sharp}^{0}$ (resp. $g_{*}^{2}$ ) of $G_{\sharp}^{0}$ (resp. of $G_{*}^{2}$ ) is equal to the Lie algebra $g_{12}$ (resp. $g_{7}$ ) in $[10]$, p. 326 327 , and that the first prolongation $\left(\mathfrak{g}_{*}^{2}\right)^{(1)}$ of $\mathfrak{g}_{*}^{2}$ in the usual sense may be identified with $\mathfrak{g}^{3}$ and the second vanishes. (Note that $\mathfrak{g}^{p}=\{0\}$ for any $p>3$.)

\section{§9. Proof of Theorem 8.1, Theorem 8.2 and of Lemma 8.2 Proof of Theorem 8.1}

9.1. Let $\left(P_{\#}^{k}, \omega^{(k)}\right)$ be a $G_{\#}^{k}$-structure on a manifold $M^{k-1}(k \geqq 0)$. $\alpha$ denotes the projection of $P_{\sharp}^{k}$ onto $M^{k-1}$. We put $P^{k}=P_{\sharp}^{k} / N^{k}$. Then $P_{\#}^{k}$ is a principal fiber bundle over the base space $P^{k}$ with structure group $N^{k}$. $\beta$ denotes the projection of $P_{\sharp}^{k}$ onto $P^{k}$. Moreover $P^{k}$ is a 
principal fiber bundle over the base space $M^{k-1}$ with structure group $G^{k}=G_{\sharp}^{k} / N^{k}$. $\sigma$ denotes the projection of $P^{k}$ onto $M^{k-1}$. We have $\alpha$ $=\sigma \circ \beta$. For any $a \in G_{\#}^{k}\left(\right.$ resp. $\left.a \in G^{k}\right), R_{\#}(a)($ resp. $R(a))$ denotes the right translation of $P_{\sharp}^{k}\left(M^{k-1}, G_{\sharp}^{k}\right)$ (resp. $\left.P^{k}\left(M^{k-1}, G^{k}\right)\right)$ induced by $a$.

Let $a \in G_{\#}^{k}$. If $k=0$, there is a $b \in G$ such that $a \equiv b\left(\bmod N^{0}\right)$, and if $k>0$, there is an $X^{k} \in \mathrm{g}^{k}$ such that $a \equiv t^{k}\left(X^{k}\right)\left(\bmod N^{k}\right)$. We have $R_{\sharp}(a)^{*} \omega^{(k)}=a^{-1} \omega^{(k)}$ and hence, according as $k=0$ or $k>0$,

$$
\begin{aligned}
& R_{\ddagger}(a)^{*}\left(\omega_{0}^{p} \equiv b^{-1} \omega_{0}^{p}\left\{\bmod \omega_{0}^{r}(r<p)\right\} \quad(p<0),\right. \\
& R_{\#}(a)^{*} \omega_{k}^{p} \equiv \omega_{k}^{p}-\left[X^{k}, \omega_{k}^{p-k}\right]\left\{\bmod \omega_{k}^{r}(r<p-k)\right\} \quad(p<k) .
\end{aligned}
$$

By (9.1), we have $R_{\sharp}(a) * D_{\# k}^{p}=D_{\# k}^{p}$ for any $a \in G_{\sharp}^{k}$ and $p<0$. In particular it follows that there is a unique differential system $D_{k}^{p}$ on $P^{k}$ such that $\beta^{*} D_{k}^{p}=D_{\# k}^{p}$. We assert that the family $D^{(k)}=\left(D_{k}^{p}\right)_{p<0}$ satisfies conditions 1) and 2) in Def. 7.2. In fact, the kernel of the linear mapping $D_{\# k}^{p}(z) \ni X \rightarrow \beta_{*} X \in D_{k}^{p}(\beta(z))$ consists of all the vertical vectors in $P_{\sharp}^{k}\left(P^{k}, N^{k}\right)$ at $z$. Therefore we have $\operatorname{dim} D_{k}^{p}=\operatorname{dim} D_{\sharp k}^{p}-\operatorname{dim} \grave{D}_{k}^{p}$. From (8.1) and the fact that $\beta^{*} D_{k}^{0}=D_{\# k}^{0}$, we get: $\cdots \supset D_{k}^{p} \supset \cdots \supset D_{k}^{-1} \supset$ $D_{k}^{0}$.

Let $a \in G_{\sharp}^{k}$ and let $\bar{a}$ be the image of a by projection of $G_{\sharp}^{k}$ onto $G^{k}$. Then we have $\beta \circ R_{\sharp}(a)=R(\bar{a}) \circ \beta$ and hence $\beta^{*}\left(R(\bar{a})^{*} D_{k}^{p}\right)=R_{\#}(a)^{*} \beta^{*} D_{k}^{p}$ $=R_{\sharp}(a) * D_{\# k}^{p}=D_{\# k}^{p}=\beta^{*} D_{k}^{p}$, whence $R(\bar{a}) * D_{k}^{p}=D_{k}^{p}$. We have thereby proved our assetion.

Let $a \in N^{k}$. By (9.1), we have $R_{\sharp}(a)^{*}\left(\omega_{k}^{p} \equiv \omega_{k}^{p}\left\{\bmod \omega_{k}^{r}(r<p-k)\right\}\right.$, whence $R_{\#}(a) *\left(\omega_{k}^{p} \mid D_{\# k}^{p-k}\right)=\omega_{k}^{p} \mid D_{\# k}^{p-k}$.

Moreover we see that $\omega_{k}^{p} \mid D_{\# k}^{p-k}$ vanishes for vertical vectors in $P_{\#}^{k}\left(P^{k}, N^{k}\right)$. Therefore we have: For any $p<k$, there is a unique $\mathfrak{g}^{p}$. valued 1 -form $\theta_{k}^{p}$ on $\left(P^{k}, D_{k}^{p-k}\right)$ such that $\beta^{*} \theta_{k}^{p}=\omega_{k}^{p} \mid D_{\# k}^{p-k}$. We must show that the family $\theta^{(k)}=\left(\theta_{k}^{p}\right)_{p<k}$ together with $D^{(k)}$ satisfies conditions 3 ) and 4) in Def. 7.2. Condition 3) is clear from the fact that, for any $p<0, D_{\# k}^{p}$ is defined by the equations $\omega_{k}^{r}=0(r<p)$ and that $D_{\# k}^{0}$ is defined by the equations $\omega_{k}^{r}=0(r<k)$. Let us verify condition 4$)$. 
First suppose $k=0$. Let $a \in G^{0} C G_{\#}^{0}$. Then we have $R(\bar{a}) \circ \beta=\beta \circ R_{\sharp}(a)$ and

$$
\begin{aligned}
\beta^{*}\left(R(\bar{a})^{*} \theta_{0}^{p}\right) & =R_{\sharp}(a)^{*} \beta^{*} \theta_{0}^{p}=R_{\sharp}(a)^{*}\left(\omega_{0}^{p} \mid D_{0}^{p}\right) \\
& =\left(a^{-1} \omega_{0}^{p}\right) \mid D_{0}^{p}=\beta^{*}\left(a^{-1} \theta_{0}^{p}\right),
\end{aligned}
$$

whence $R(a)^{*} \theta_{0}^{p}=a^{-1} \theta_{0}^{p}$. Now suppose $k>0$. Let $a \ni G_{\sharp}^{k}$ and let $X^{k}$ be as before. Then we have $\bar{a}=\exp X^{k}$ and $R(\bar{a}) \circ \beta=\beta \circ R_{\sharp}(a)$. By using (9.1), we have

$$
\begin{aligned}
\beta^{*}\left(R(\bar{a})^{*} \theta_{k}^{p}\right) & =R_{\#}(a)^{*} \beta^{*} \theta_{k}^{p}=R_{\sharp}(a)^{*}\left(\omega_{k}^{p} \mid D_{\sharp k}^{p-k}\right) \\
& =\omega_{k}^{p} \mid D_{\# k}^{p-k}-\left[X^{k}, \omega_{k}^{p-k} \mid D_{\# k}^{p-k}\right] \\
& =\beta^{*}\left(\theta_{k}^{p}-\left[X^{k}, \theta_{k}^{p-k} \mid D_{k}^{p-k}\right]\right),
\end{aligned}
$$

whence $R(\bar{a})^{*} \theta_{k}^{p}=\theta_{k}^{p}-\left[X^{k}, \theta_{k}^{p-k} \mid D_{k}^{p-k}\right]$.

We have thereby proved that the system $\left(P^{k}, D^{(k)}, \theta^{(k)}\right)$ is a pseudo$G^{k}$-structure on $M^{k-1}$.

Assume that the $G_{\sharp}^{k}$-structure $\left(P_{\sharp}^{k}, \omega^{(k)}\right)$ is of type $n$. Since $N^{k}$ is homeomorphic with a Euclidean space, $P \sharp\left(P^{k}, N^{k}\right)$ admits a cross-section g. Putting $\xi^{(k)}=g^{*}\left(\omega^{(k)}\right.$, then we have $g^{*} D_{k}^{p}(y) \subset D_{\# k}^{p}(g(y))$ and $\xi_{k}^{p}(X)$ $=\omega_{k}^{p}\left(g_{*} X\right)=\left(\beta^{*} \theta_{k}^{p}\right)\left(g_{*} X\right)=\theta_{k}^{p}(X)$ for any $y \in P^{k}, X \in D_{k}^{p-k}(y)$ and $p$ $<0$. This shows that $\xi^{(k)}$ is compatible with $\theta^{(k)}$. Moreover it is clear from the equalities in Def. 7.1 that $\xi^{(k)}$ satisfies the equalities in Def. 7.5. Thus we have proved that the pseudo- $G^{k}$-structure $\left(P^{k}, D^{(k)}, \theta^{(k)}\right)$ is of type $\mathrm{m}$.

9.2. We shall show that the assignment $\left(P_{\sharp}^{k}, \omega^{(k)}\right) \rightarrow\left(P^{k}, D^{(k)}, \theta^{(k)}\right)$ is compatible with the respective isomorphisms. Let $\left(P_{\#}^{k}, \omega^{(k)}\right)$ (resp.

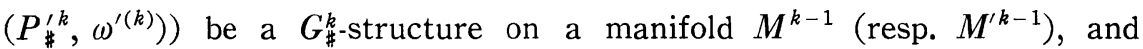
let $\left(P^{k}, D^{(k)}, \theta^{(k)}\right)$ (resp. $\left(P^{\prime k}, D^{\prime(k)}, \theta^{\prime(k)}\right)$ ) be the corresponding pseudo$G^{k}$-structure on $M^{k-1}$ (resp. $M^{\prime k-1}$ ). We shall write as $A^{\prime}$ the quantity in $\left(P_{\sharp}^{\prime k}, \omega^{\prime(k)}\right)$ or $\left(P_{\sharp}^{\prime k}, D^{\prime(k)}, \theta^{\prime(k)}\right)$ which corresponds to a quantity $A$ in $\left(P_{\#}^{k}, \omega^{(k)}\right)$ or $\left(P^{k}, D^{(k)}, \theta^{(k)}\right)$. 
Every isomorphism $\varphi_{\#}$ of $\left(P_{\sharp}^{k}, \omega^{(k)}\right)$ onto $\left(P_{\#}^{\prime}, \omega^{\prime(k)}\right)$ induces a bundle isomorphism $\varphi$ of $P^{k}\left(M^{k-1}, G^{k}\right)$ onto $P^{\prime k}\left(M^{\prime k-1}, G^{k}\right)$. We have $\varphi \circ \beta$ $=\beta^{\prime} \circ \varphi_{\sharp}$. Since $\varphi_{\#}^{*} \omega^{\prime(k)}=\omega^{(k)}$, we see that $\varphi^{*} D_{k}^{\prime p}=D_{k}^{p}(p<0)$ and $\varphi^{*} \theta_{k}^{\prime p}$ $=\theta_{k}^{p}(p<k)$. Thus $\varphi$ gives an isomorphism of $\left(P^{k}, D^{(k)}, \theta^{(k)}\right)$ onto $\left(P^{\prime k}\right.$, $\left.D^{\prime(k)}, \theta^{\prime(k)}\right)$.

Conversely, we assume that there is given an isomorphism $\varphi$ of $\left(P^{k}, D^{(k)}, \theta^{(k)}\right)$ onto $\left(P^{\prime k}, D^{\prime(k)}, \theta^{\prime(k)}\right)$.

First, suppose that there is a mapping $\psi$ of $P_{\#}^{k}$ to $P_{\#}^{\prime k}$ such that $\varphi \circ \beta=\beta^{\prime} \circ \psi$. Since $\varphi^{*} D_{k}^{\prime p}=D_{k}^{p}(p<0)$ and $\varphi^{*} \theta_{k}^{\prime p}=\theta_{k}^{p}(p<k)$, we have

$$
\begin{aligned}
& \psi^{*} D_{\sharp k}^{\prime p}=D_{\sharp k}^{p} \quad(p<0), \\
& \left(\psi^{*} \omega_{k}^{\prime p}-\omega_{k}^{p}\right) \mid D_{\sharp k}^{p-k}=0 \quad(p<k) .
\end{aligned}
$$

Next, suppose that there are two mappings $\psi_{1}$ and $\psi_{2}$ of $P_{\#}^{k}$ to $P_{\#}^{\prime k}$ such that $\varphi \circ \beta=\beta^{\prime} \circ \psi_{1}=\beta^{\prime} \circ \psi_{2}$. Then there is a unique mapping $\sigma$ of

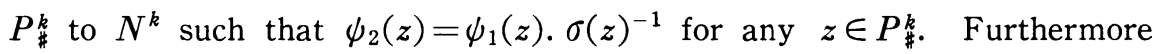
there is a unique mapping $u$ of $P_{\sharp}^{k}$ to $u^{k}$ such that $\sigma(z)=t^{k}(u(z))$ for any $z \in P_{\#}^{k}$. Let $u^{p}$ denote the ${ }^{k p}{ }^{k p}$-component of $u$ in the decomposition $\mathfrak{l t}^{k}=\sum_{p<k} \mathfrak{n}^{k p}$. These being said, we have $\sigma \cdot \psi_{1}^{*} \omega^{\prime(k)}=\phi_{2}^{*} \omega^{\prime(k)}$ and hence

$$
\psi_{2}^{*} \omega_{k}^{\prime p}=\psi_{1}^{*} \omega_{k}^{\prime p}+u_{r<p-k}^{p}\left(\sum_{1} \psi_{1}^{*} \omega_{k}^{\prime r}\right) \quad(p<k) .
$$

By using these facts, we shall prove that there exists a unique isomorphism $\varphi_{\#}$ of $\left(P_{\#}^{k}, \omega^{(k)}\right)$ onto $\left(P_{\#}^{\prime k}, \omega^{\prime(k)}\right)$ which induces the given $\varphi$.

First of all, let us prove uniqueness of $\varphi_{\#}$. Suppose that there are two isomorphisms $\phi_{1}$ and $\psi_{2}$ of $\left(P_{\sharp}^{k}, \omega^{(k)}\right)$ onto $\left(P_{\#}^{\prime k}, \omega^{\prime(k)}\right)$ such that $\varphi \circ \beta=\beta^{\prime} \circ \psi_{1}=\beta^{\prime} \circ \psi_{2}$. Since $\psi_{1}^{*} \omega^{\prime(k)}=\psi_{2}^{*} \omega^{\prime(k)}=\omega^{(k)}$, it follows from (9.3) that $u_{r<p-k}^{p}\left(\sum_{k}\right)=0$, whence $u^{p}=0$. Therefore we get $\sigma(z)=e$ for any $z \in P$. Thus $\psi_{1}$ and $\psi_{2}$ must coincide.

Let us now prove existence of $\varphi_{\#}$. By uniqueness of $\varphi_{\#}$ just proved, we may assume without loss of generality that both $P_{\#}^{k}\left(M^{k-1}\right.$, $\left.G_{\sharp}^{k}\right)$ and $P_{\sharp}^{\prime k}\left(M^{\prime k-1}, G_{\sharp}^{k}\right)$ are trivial. It follows that there is a mapping 
$\psi$ of $P_{\#}^{k}$ to $P_{\#}^{\prime k}$ such that $\varphi \circ \beta=\beta^{\prime} \circ \psi$. By (9.2), we have $\psi^{*} \omega_{k}^{\prime r}$ $=\omega_{k}^{r}(r<k-\mu) . \quad p$ being an integer $<k$, suppose that there is a mapping $\psi_{p-1}$ of $P_{\sharp}^{k}$ to $P_{\#}^{\prime k}$ such that $\phi \circ \beta=\beta^{\prime} \circ \psi_{p-1}$ and such that $\psi_{p-1}^{*} \omega_{k}^{\prime r}$ $=\omega_{k}^{r}(r<p)$. Then by $(9.2)$, we have $\psi_{p-1}^{*} \omega_{k}^{\prime p}-\omega_{k}^{p} \equiv 0\left\{\bmod \omega_{k}^{r}(r<p-k)\right\}$. Therefore we can find a unique mapping $u^{p}$ of $P_{\#}^{k}$ to $u^{k p}$ satisfying the equality:

$$
\omega_{k}^{p}=\psi_{p-k}^{*} \omega_{k}^{\prime p}+u^{p}\left(\sum_{r<p-k} \omega_{k}^{r}\right)
$$

Define a mapping $\sigma$ of $P_{\sharp}^{k}$ to $N^{k}$ by $\sigma(z)=t^{k}\left(u^{p}(z)\right)$ for any $z \in P_{\sharp}^{k}$, and a mapping $\psi_{p}$ of $P_{\#}^{k}$ to $P_{\#}^{\prime k}$ by $\psi_{p}(z)=\psi_{p-1}(z) \cdot \sigma(z)^{-1}$ for any $z$ $\in P$ \#. Then we clearly have $\varphi \circ \beta=\beta^{\prime} \circ \psi_{p}$. From (9.3) and (9.4), we get $\psi_{p}^{*} \omega_{k}^{\prime r}=\omega_{k}^{r}(r \leqq p)$. Therefore by induction we know that there is a mapping $\varphi_{\#}$ of $P_{\#}^{k}$ to $P_{\#}^{\prime k}$ such that $\varphi \circ \beta=\beta^{\prime} \circ \varphi_{\#}$ and $\varphi_{\#}^{*} \omega^{(k)}=\omega^{(k)}$. It can be easily shown that $\varphi_{\#}$ gives a bundle isomorphism of $P_{\#}^{k}\left(M^{k-1}\right.$, $\left.G_{\sharp}^{k}\right)$ onto $P_{\sharp}^{\prime k}\left(M^{\prime k-1}, G_{\sharp}^{k}\right)$. Thus $\varphi_{\sharp}$ is an isomorphism of $\left(P_{\sharp}^{k}, \omega^{(k)}\right)$ onto $\left(P_{\#}^{\prime k}, \omega^{\prime(k)}\right)$ which induces the given $\varphi$.

We have thereby proved Th. 8.1.

\section{Proof of Theorem 8.2}

9.3. Let $\left(P^{k-1}, D^{(k-1)}, \theta^{(k-1)}\right)$ be a pseudo- $G^{k-1}$-structure on a manifold $M^{k-2}(k \geqq 1)$. $\sigma$ denotes the projection of $P^{k-1}$ onto $M^{k-2}$. For any $a \in G^{k-1}$ (resp. $X \in \mathrm{g}^{k-1}$ ), $R(a)$ (resp. $r(X)$ ) denotes the right translation (resp. the vertical vector field) of $P^{k-1}$ induced by a (resp. $X)$.

We have $\operatorname{dim} P^{k-1}=\operatorname{dim} \mathrm{m}^{k-1}$. Considering the Lie subgroup $H^{k}$ of $G L\left(\mathrm{~m}^{k-1}\right)$, let us define a $H^{k}$-structure $\left(F^{k}, \omega^{(k)}\right)$ on $P^{k-1}$ as follows: First of all, by using the vector space $\mathrm{m}^{k-1}$, we define the frame bundle $\tilde{F}$ of $P^{k-1}$ as in [13], $\S 1$. Let $\tilde{\pi}$ be the projection of $\tilde{F}$ onto $P^{k-1}$. Then we define $F^{k}$ to be the subset of $\tilde{F}$ which consists of all the elements $z$ satisfying the following conditions:
1) $z \cdot \mathrm{\triangleright}_{k-1}^{p}=D_{k-1}^{p}(\tilde{\pi}(z))(p<0)$;
2) $z \cdot Z^{k-1}=r\left(Z^{k-1}\right)_{\tilde{\pi}(z)}$ for any $Z^{k-1} \in \mathfrak{g}^{k-1}$; 
3) $\theta_{k-1}^{p}(z Z)=\varepsilon_{k-1}^{p}(Z)$ for any $Z \in \aleph_{k-1}^{p-k ! 1}(p \leqq k-2)$.

It can be easily shown that $F^{k}$ is a $H^{k}$-subbundle of $\tilde{F}$. Thus we have obtained a $H^{k}$-structure $\left(F^{k}, \omega^{(k)}\right)$ on $P^{k-1}, \omega^{(k)}$ being the basic form of $F^{k}$. $\pi$ denotes the projection of $F^{k}$ onto $P^{k-1}$. For any $a \in H^{k}, \bar{R}(a)$ denotes the right translation of $F^{k}$ induced by $a$. Moreover, denote by $\omega_{k}^{p}$ the $\mathrm{g}^{p}$-component of $\omega^{(k)}$ in the decomposition $m^{k-1}=\sum g^{p}$ and, for each $p<0$, denote by $\bar{D}_{k}^{p}$ the differential system on $F^{k}$ defined by the equations $\omega_{k}^{r}=0(r<p)$. We have $\cdots \supset \bar{D}_{k}^{p} \cdots \supset \bar{D}_{k}^{-1} \supset \bar{D}_{k}^{0}$, where $\bar{D}_{k}^{0}$ denotes the differential system on $F^{k}$ consisting of all the vertical vectors in $F^{k}\left(P^{k-1}, H^{k}\right)$. We have $\operatorname{dim} \bar{D}_{k}^{p}=\operatorname{dim} \mathfrak{b}_{k}^{p}+\operatorname{dim} \mathfrak{h}^{k}(p<0)$.

Lemma 9.1. $\pi^{*} D_{k-1}^{p}=\bar{D}_{k}^{p}(p<0)$,

$$
\pi^{*} \theta_{k-1}^{p}=\omega_{k}^{p} \mid \bar{D}_{k}^{p-k+1}(p<k-1)
$$

Proof. Let $z \in F^{k}$ and $Z \in T_{z}\left(F^{k}\right)$. Then we have $\pi_{*} Z=z \cdot \omega^{(k)}(Z)$ $=\sum_{p<k} z \cdot \omega_{k}^{p}(Z)$. It follows that $Z \in\left(\pi^{*} D_{k-1}^{p}\right)(z)$ if and only if $Z$ $\in \bar{D}_{k}^{p}(z)$, whence $\pi^{*} D_{k-1}^{p}=\bar{D}_{k}^{p}$. Let $Z \in \bar{D}_{k}^{p-k+1}(z)$. Then we have $\pi_{*} Z$ $=\sum_{r=p-k+1}^{k-1} z \cdot \omega_{k}^{r}(Z)$. Therefore we get $\left(\pi^{*} \theta_{k-1}^{p}\right)(Z)=\sum_{r=p-k+1}^{k-1} \varepsilon_{k-1}^{p}\left(\omega_{k}^{r}(Z)\right)$ $=\omega_{k}^{p}(Z)$, whence $\pi^{*} \theta_{k-1}^{p}=\omega_{k}^{p} \mid \bar{D}_{k}^{p-k+1}$.

Assume for a moment that $k=1$. Every element $a$ of $G^{0}$ is extended to a unique automorphism, denoted by the same letter $a$, of the graded Lie algebra g. In particular, it follows that the group $G^{0}$ is represented on the vector space $\mathrm{m}^{0}$. For any $z \in F^{1}$ and $a \in G^{0}$, define an element $z a$ of $\tilde{F}$ by $(z a) \cdot Z=R(a)_{*}(z \cdot(a Z))$ for any $Z \in u^{0}$.

Lemma 9.2. Let $z \in F^{1}$ and $a, b \in G^{0}$.

(1) $z a \in F^{1}$.

(2) $(z a) b=z(a b)$.

(3) $\pi(z a)=\pi(z) a$.

(4) $\hat{R}(a)^{*} \omega^{(1)}=a^{-1} \omega^{(1)}$, where $\hat{R}(a)$ denotes the transformation $F^{1} \ni z \rightarrow z a \in F^{1}$. 
Proof. (2) and (3) are clear. (1) $(z a) \cdot \S_{0}^{p}=R(a)_{*}\left(z \cdot \S_{0}^{p}\right)=R(a)_{*}$ $D_{0}^{p}(\pi(z))=D_{0}^{p}(\tilde{\pi}(z a))$. Let $Z^{0} \in \mathfrak{g}^{0} . \quad(z a) Z^{0}=R(a)_{*}\left(z \cdot\left(a Z^{0}\right)\right)=R(a)_{*}$ $r\left(a Z^{0}\right)_{\pi(z)}=r\left(a^{-1} a Z\right)_{\tilde{\pi}(z a)}=r\left(Z^{0}\right)_{\tilde{\pi}(z a)}$. Let $Z \in \mathbb{D}_{0}^{p} . \quad \theta_{0}^{p}((z a) \cdot Z)=a^{-1} \theta_{0}^{p}(z \cdot$ $a Z))=a^{-1} \varepsilon_{0}^{p}(a Z)=a^{-1} a \varepsilon_{0}^{p}(Z)=\varepsilon_{0}^{p}(Z)$. Thus we get $z a \in F^{1}$.

(4) Let $Z \in T_{z}\left(F^{1}\right) . \quad \pi_{*} \hat{R}(a)_{*} Z=(z a) \cdot\left(\hat{R}(a)^{*} \omega^{(1)}\right)(Z) . \quad \pi_{*} \hat{R}(a)_{*} Z$ $=R(a)_{*} \pi_{*} Z=R(a)_{*}\left(z \cdot \omega^{(1)}(Z)\right)=(z a) \cdot\left(a^{-1} \omega^{(1)}(Z)\right)$.

Hence, $\hat{R}(a)^{*} \omega^{(1)}=a^{-1} \omega^{(1)}$.

For any $X^{0} \in \mathfrak{g}^{0}$, let $\hat{r}\left(X^{0}\right)$ denote the vector field on $F^{1}$ which is induced by the one parameter group of transformations of $F^{1}$, $\hat{R}\left(\exp t X^{0}\right)$.

Lemma 9.3. Let $X^{0} \in \mathrm{g}^{0}$.

(1) $\pi_{*} \hat{r}\left(X^{0}\right)_{z}=r\left(X^{0}\right)_{\pi(z)}$ at each $z \in F^{1}$.

(2) $\omega_{1}^{p}\left(\hat{r}\left(X^{0}\right)\right)=\delta_{p, 0} X^{0}(p \leqq 0)$.

(3) $L_{\widehat{r}\left(X^{0}\right)} \omega_{1}^{p}+\left[X^{0}, \omega_{1}^{p}\right]=0 \quad(p \leqq 0)$.

Proof. (1) and (3) follow immediately from Lemma 9.2, (3) and (4). (2) $\pi_{*} \hat{r}\left(X^{0}\right)_{z}=z \cdot \omega^{(1)}\left(\hat{r}\left(X^{0}\right)_{z}\right)$ and $\pi_{*} \hat{r}\left(X^{0}\right)_{z}=r\left(X^{0}\right)_{\pi(z)}=z \cdot X^{0}$. Therefore we get $\omega^{(1)}\left(\hat{r}\left(X^{0}\right)\right)=X^{0}$.

Now, assume for a moment that $k>1$. For any $a \in G^{k-1}$ and $Z$ $\in \mathrm{m}^{k-1}$, define an element $a Z$ of $\mathfrak{m}^{k-1}$ by

$$
a Z=Z+\sum_{p<0}\left[X^{k-1}, Z^{p}\right]
$$

where $X^{k-1}$ is a unique element of $\mathrm{g}^{k-1}$ such that $a=\exp X^{k-1}$. We clearly have $e Z=Z$. Let $a, b \in G^{k-1}$ and express them respectively as $\exp X^{k-1}$, exp $Y^{k-1}$, where $X^{k-1}, Y^{k-1} \in \mathrm{g}^{k-1}$. If $1<k \leqq \mu$ then there is a unique element $w$ of $\mathfrak{u t}^{k}$ such that $a(b Z)=(a b) t^{k}(w) Z$ for any $Z$ $\in \mathrm{m}^{k-1}$. If $k>\mu$, then we have $a(b Z)=(a b) Z$ for any $Z \in \mathrm{m}^{k-1}$, i.e., the group $G^{k-1}$ is represented on $\mathrm{m}^{k-1}$. For any $z \in F^{k}$ and $a \in G^{k-1}$, define an element $z a$ of $\tilde{F}$ by $(z a) \cdot Z=R(a)_{*}(z \cdot(a Z))$ for all $Z \in \mathrm{m}^{k-1}$.

Lemma 9.4. Let $z \in F^{k}$ and $a, b \in G^{k-1}$. 
(1) $z a \in F^{k}$.

(2) $\quad(z a) b=z(a b) t^{k}(w) \quad(1 \leqq k \leqq \mu)$, $(z a) b=z(a b) \quad(\mu<k)$.

(3) $\pi(z a)=\pi(z) a$

(4) $a\left(\hat{R}(a)^{*} \omega^{(k)}\right)=\omega^{(k)}$, where $\hat{R}(a)$ denotes the transformation $F^{k} \ni z \rightarrow z a \in F^{k}$.

Proof. (2)-(4) are just analogous to (2)-(4) in Lemma 9.2. Let us prove (1). $(z a) \cdot \diamond_{k-1}^{p}=R(a)_{*}\left(z \cdot \diamond_{k-1}^{p}\right)=R(a)_{*} D_{k-1}^{p}(\pi(z))=D_{k-1}^{p}(\tilde{\pi}(z a))$. Let $Z^{k-1} \in \mathrm{g}^{k-1}$. $(z a) \cdot Z^{k-1}=R(a)_{*}\left(z \cdot Z^{k-1}\right)=R(a)_{*} r\left(Z^{k-1}\right)_{\pi(z)}=r\left(Z^{k-1}\right)_{\tilde{\pi}(z a)}$. This last equality is the case, because $G^{k-1}$ is an abelian group. Let $Z \in \mathfrak{D}_{k-1}^{p-k+1}$.

$$
\begin{aligned}
& \theta_{k-1}^{p}((z a) \cdot Z)=\left(R(a)^{*} \theta_{k-1}^{p}\right)(z \cdot(a Z)) \\
& \quad=\left(\theta_{k-1}^{p}-\left[X^{k-1}, \theta_{k-1}^{p-k+1} \mid D_{k-1}^{p-k+1}\right]\right)\left(z \cdot\left(Z+\sum_{q<0}\left[X^{k-1}, Z^{q}\right]\right)\right) \\
& \quad=\varepsilon_{k-1}^{p}\left(Z+\sum_{q<0}\left[X^{k-1}, Z^{q}\right]\right)-\left[X^{k-1}, \varepsilon_{k-1}^{p-k+1}\left(Z+\sum_{q<0}\left[X^{k-1}, Z^{q}\right]\right)\right] \\
& \quad=Z^{p}+\left[X^{k-1}, Z^{p-k+1}\right]-\left[X^{k-1}, Z^{p-k+1}\right]-\left[X^{k-1},\left[X^{k-1}, Z^{p-2 k+2}\right]\right] .
\end{aligned}
$$

Since $p-2 k+2<p-k+1$, we have $Z^{p-2 k+2}=0$ and hence $\theta_{k-1}^{p}((z a) \cdot Z)$ $=Z^{p}=\varepsilon_{k-1}^{p}(Z)$. Thus we have proved $z a \in F^{k}$.

Let $X^{k-1} \in \mathfrak{g}^{k-1}$ and put $f_{t}=\hat{R}\left(\exp t X^{k-1}\right)$. Then we see that $f_{t}$ is a one parameter family of transformations of $F^{k}$ and that $f_{0}$ is the identity transformation. We denote by $\hat{r}\left(X^{k-1}\right)$ the vector field on $F^{k}$ induced by the family $f_{t}$, i.e., $\hat{r}\left(X^{k-1}\right)_{z}=\frac{\partial f_{t}(z)}{\partial t}{ }_{t=0}$.

Lemma 9.5. Let $X^{k-1} \in \mathrm{g}^{k-1}$.

(1) $\pi_{*} \hat{r}\left(X^{k-1}\right)_{z}=r\left(X^{k-1}\right)_{\pi(z)}$ at each $z \in F^{k}$.

(2) $\omega_{k}^{p}\left(\hat{r}\left(X^{k-1}\right)\right)=\delta_{p, k-1} X^{k-1}(p \leqq k-1)$.

(3) $L_{\hat{\boldsymbol{r}}\left(X^{k-1}\right)} \omega_{k}^{p}+\left[X^{k-1}, \omega_{k}^{p-k+1}\right]=0 \quad(p<k-1)$, 


$$
L_{\widehat{r}\left(X^{k-1}\right)} \omega_{k}^{k-1}=0 .
$$

Proof. This follows from Lemma 9.4. The proof is just analogous to that of Lemma 9.3.

Let us return to the general case. By using the basic form $\omega^{(k)}$ $=\sum_{p<k} \omega_{k}^{p}$, we define $\mathfrak{g}^{p}$-valued 2 -forms $\Omega_{k}^{p}$ on $F^{k}$ as in Def. 7.1. i.e.,

$$
\Omega_{k}^{p}=d \omega_{k}^{p}+\frac{1}{2} \sum_{r+s=p}\left[\omega_{k}^{r}, \omega_{k}^{s}\right] \quad(p \leqq k-2) .
$$

Lemma 9.6. For any $p \leqq k-2$, we have

$$
\Omega_{k}^{p} \equiv 0\left\{\bmod \omega_{k}^{r}(r \leqq p-k) ; \omega_{k}^{r} \wedge \omega_{k}^{s}(p-k<r, s<k)\right\}
$$

Proof. This is easy from existence of a connection in $F^{k}\left(P^{k-1}\right.$, $\left.H^{k}\right)$.

9.4. In what follows, we assume that the pseudo- $G^{k-1}$-structure $\left(P^{k-1}, D^{(k-1)}, \theta^{(k-1)}\right)$ is of type $n t$.

Lemma 9.7. For any $p \leqq k-3$, we have

$$
\Omega_{k}^{p} \equiv 0\left\{\bmod \omega_{k}^{r}(r \leqq p-k+1) ; \omega_{k}^{r} \wedge \omega_{k}^{s}((r, s) \in I(k-1, p))\right\}
$$

Proof. By the assumption, there is an $\mathrm{m}^{k-2}$-valued 1 -form $\xi^{(k-1)}$ on $P^{k-1}$ which is compatible with $\theta^{(k-1)}$ and which satisfies the equalities (with $k$ replaced by $k-1$ ) in Def. 7.5. Therefore if we put $\xi^{p}=\pi^{*} \xi_{k}^{p}$, then we have

$$
\begin{aligned}
& d \bar{\xi}^{p}+\frac{1}{2} \sum_{r+s=p}\left[\bar{\xi}^{r}, \bar{\xi}^{s}\right] \equiv 0 \\
& \left\{\bmod \bar{\xi}^{r}(r \leqq p-k+1) ; \bar{\xi}^{r} \wedge \bar{\xi}^{s}((r, s) \in I(k-1, p))\right\} \quad(p \leqq k-3) .
\end{aligned}
$$

Moreover by Lemma 9.1. we have $\left(\bar{\xi}^{p}-\omega_{k}^{p}\right) \mid \bar{D}_{k}^{p-k+1}=0(p \leqq k-2)$ and hence

$$
\bar{\xi}^{p} \equiv \omega_{k}^{p}\left\{\bmod \omega_{k}^{r}(r \leqq p-k)\right\}
$$


On differential systems, graded Lie algebras and pseudo-groups

$$
\omega_{k}^{p} \equiv \xi^{p}\left\{\bmod \bar{\xi}^{r}(r \leqq p-k)\right\} \quad(p \leqq k-2) .
$$

Lemma 9.7 follows easily from (9.5) and (9.6).

Lemma 9.8. For any $p \leqq k-2$, we have

$$
\Omega_{k}^{p} \equiv 0\left\{\bmod \omega_{k}^{r}(r \leqq p-k) ; \omega_{k}^{r} \wedge\left(\omega_{k}^{s}((r, s) \in I(k, p))\right\}(p \leqq k-2) .\right.
$$

Proof. Let $X^{k-1} \in \mathrm{g}^{k-1}$. By Lemmas 9.3 and 9.5, we have

$$
\left.\hat{r}\left(X^{k-1}\right)\right\lrcorner \omega_{k}^{p}=\delta_{p, k-1} X^{k-1}(p \leqq k-1) .
$$

Moreover we have

$$
\left.\hat{r}\left(X^{k-1}\right)\right\lrcorner \Omega_{k}^{p}=0 \quad(p \leqq k-2),
$$

because

$$
\begin{aligned}
& L_{\widehat{r}\left(X^{k-1}\right)} \omega_{k}^{p}+\left[X^{k-1}, \omega^{p-k+1}\right]=0 \text { (Lemmas } 9.3 \text { and 9.5). } \\
& \left.\left.L_{\widehat{r}\left(X^{k-1}\right)} \omega_{k}^{p}=\hat{r}\left(X^{k-1}\right)\right\lrcorner d \omega_{k}^{p}+d\left(\hat{r}\left(X^{k-1}\right)\right\lrcorner \omega_{k}^{p}\right), \\
& \left.\left.\left.\hat{r}\left(X^{k-1}\right)\right\lrcorner \Omega_{k}^{p}=\hat{r}\left(X^{k-1}\right)\right\lrcorner d \omega_{k}^{p}+\sum_{r+s=p}\left[\hat{r}\left(X^{k-1}\right)\right\lrcorner \omega_{k}^{r}, \omega_{k}^{s}\right] .
\end{aligned}
$$

Let $p$ be any integer $\leqq k-3$. From Lemmas 9.6 and 9.7 , it follows that

(9.9) $\Omega_{k}^{p} \equiv 0\left\{\bmod \omega_{k}^{r}(r \leqq p-k) ; \omega_{k}^{p-k+1} \wedge \omega_{k}^{k-1} ; \omega_{k}^{r} \wedge \omega_{k}^{s}((r, s) \in I(k, p))\right\}$.

Then by (9.7), (9.8) and (9.9), we get the equality in Lemma 9.8 for $p \leqq k-3$. From Lemma 9.6 , it follows that

$$
\begin{array}{r}
\Omega_{k}^{k-2} \equiv 0\left\{\bmod \omega_{k}^{r}(r \leqq-2) ; \omega_{k}^{k-1} \wedge \omega_{k}^{r}(-1 \leqq r \leqq k-1) ;\right. \\
\left.\omega_{k}^{r} \wedge \omega_{k}^{s}((r, s) \in I(k, k-2))\right\}
\end{array}
$$

Then by (9.7), (9.8) and (9.10), we get the equality in Lemma 9.8 for $p=k-2$. 
Let $p$ be any integer $\leqq k-3$. Let $J(p)$ be the sebset of $I(k, p)$ consisting of all the pairs $(r, s) \in \boldsymbol{Z} \times \boldsymbol{Z}$ such that $r=p-k+1,-1 \leqq s$ $\leqq k-2$ or $-1 \leqq r \leqq k-2, s \leqq p-k+1$. We put $I_{*}(k, p)=I(k, p)$ $-J(p)$. By Lemma 9.8, then we see that there is a unique mapping $C^{p}$ of $F^{k}$ to $\mathfrak{S}_{k-1}^{p}$ satisfying the equality

$$
\Omega_{k}^{p}+C^{p}\left(\alpha \wedge \omega_{k}^{p-k+1}\right) \underset{p}{\equiv 0}
$$

where $\alpha=\sum_{r=-1}^{k-2} \omega_{k}^{r}$ and where, in general, $A \equiv \underset{p}{\equiv} B$ means that $A \equiv B\{\bmod$ $\left.\omega_{k}^{r}(r \leqq p-k) ; \omega_{k}^{r} \wedge \omega_{k}^{s}\left((r, s) \in I_{*}(k, p)\right)\right\}$. Moreover by Lemma 9.8 , there is a unique mapping $C^{k-2}$ of $F^{k}$ to $\mathfrak{S}_{k-1}^{k-2}$ satisfying the equality:

$$
\Omega_{k}^{k-2}+\frac{1}{2} C^{k-2}(\alpha \wedge \alpha) \underset{k-2}{\equiv 0}
$$

where, in general, $A \equiv B$ means that $A \equiv B\left\{\bmod \omega_{k}^{r}(r \leqq-2)\right\}$. We put $C=\sum_{p \leqq k-2} C^{p}$, which gives a mapping of $F^{k}$ to $C^{(k-1)}$.

Lemma 9.9. Let $a \in H^{k}$ and let $u \in \mathfrak{g}^{k}$ be such that $a \equiv t^{k}(u)(\bmod$ $\left.N^{k}\right)$. Then we have

$$
\bar{R}(a) * C=C+\partial u \text {. }
$$

Proof. Since $\bar{R}(a)^{*} \omega^{(k)}=a^{-1}()^{(k)}$, we have

$$
\begin{aligned}
& \bar{R}(a)^{*} \omega_{k}^{p} \equiv \omega_{k}^{p}-u\left(\omega_{k}^{p-k}\right)\left\{\bmod \omega_{k}^{r}(r<p-k)\right\} \quad(p \leqq k-2) . \\
& \bar{R}(a)^{*} \omega_{k}^{k-1} \equiv \omega_{k}^{k-1}-u(\alpha)\left\{\bmod \omega_{k}^{r}(r \leqq-2)\right\} .
\end{aligned}
$$

By Lemma 9.8, we have

$$
\begin{aligned}
& d \omega_{k}^{p}+\frac{1}{2} \sum_{\substack{r+s=0 \\
r, s<0}}\left[\omega_{k}^{r}, \omega_{k}^{s}\right] \equiv 0 \\
& \left\{\bmod \omega_{k}^{r}(r \leqq p) ; \omega_{k}^{r} \wedge \omega_{k}^{s}((r, s) \in I(0, p))\right\} \quad(p \leqq-2) .
\end{aligned}
$$

Let $p$ be any integer $\leqq k-3$. From (9.11), (9.13) and (9.14), we 
get the following equalities:

$$
\begin{aligned}
& \bar{R}(a)^{*}\left(\Omega_{k}^{p}+C\left(\alpha \wedge \omega_{k}^{p-k+1}\right)\right) \underset{p}{\equiv} 0 \\
& \bar{R}(a)^{*} d \omega_{k}^{p} \equiv d \omega_{k}^{p}+u\left(\left[\omega_{k}^{-1}, \omega_{k}^{p-k+1}\right]\right), \\
& \left.\frac{1}{2} \bar{R}(a)_{r+s=p}^{*}\left(\sum_{k}\left[\omega_{k}^{r}, \omega_{k}^{s}\right]\right) \underset{p}{\equiv} \frac{1}{2} \underset{r+s=p}{ }\left(\omega_{k}^{r}, \omega_{k}^{s}\right]\right)-\left[u(\alpha), \omega_{k}^{p-k+1}\right] \\
& \left.-\left[u\left(\omega_{k}^{p-k+1}\right), \omega_{k}^{-1}\right]\right), \\
& \bar{R}(a)^{*}\left(C\left(\alpha \wedge \omega_{k}^{p-k+1}\right)\right) \underset{p}{\equiv}\left(\bar{R}(a)^{*} C\right)\left(\alpha \wedge \omega_{k}^{p-k+1}\right) .
\end{aligned}
$$

Therefore we have

$$
\begin{aligned}
& -C\left(\alpha \wedge \omega_{k}^{p-k+1}\right)+(\bar{R}(a) * C)\left(\alpha \wedge \omega_{k}^{p-k+1}\right) \\
& +u\left(\left[\omega_{k}^{-1}, \omega_{k}^{p-k+1}\right]\right)-\left[u(\alpha), \omega_{k}^{p-k+1}\right] \\
& -\left[u\left(\omega_{k}^{p-k+1}\right), \omega_{k}^{-1}\right] \underset{p}{\equiv} 0 .
\end{aligned}
$$

“ $\underset{p}{\equiv} "$ in this last equality clearly reduces to " $=0 "$. Thus we get

$$
\begin{aligned}
& -C(X \wedge Y)+(\bar{R}(a) * C)(X \wedge Y)+u\left(\left[X^{-1}, Y\right]\right) \\
& -[u(X), Y]+\left[u(Y), X^{-1}\right]=0
\end{aligned}
$$

for any $X \in \mathfrak{b}_{k-2}^{-1}$ and $Y \in \mathfrak{g}^{p-k+1}$.

From (9.12), (9.13) and (9.14), we get the following equalities:

$$
\begin{aligned}
& \bar{R}(a) *\left(\Omega_{k}^{k-2}+\frac{1}{2} C(\alpha \wedge \alpha)\right) \underset{k-2}{\equiv} . \\
& \bar{R}(a)^{*} d \omega_{k}^{k-2} \underset{k-2}{\equiv} d \omega_{k}^{k-2}+\frac{1}{2} u\left(\left[\omega_{k}^{-1}, \omega_{k}^{-1}\right]\right) . \\
& \bar{R}(a) *\left[\omega_{k}^{k-1}, \omega_{k}^{-1}\right]_{k-2} \equiv\left[\omega_{k}^{k-1}, \omega_{k}^{-1}\right]-\left[u(\alpha), \omega_{k}^{-1}\right] .
\end{aligned}
$$




$$
\frac{1}{2} \bar{R}(a)^{*}(C(\alpha \wedge \alpha)) \underset{k-2}{\equiv} \frac{1}{2}(\bar{R}(a) * C)(\alpha \wedge \alpha)
$$

Therefore we have

$$
\begin{aligned}
& -\frac{1}{2} C(\alpha \wedge \alpha)+\frac{1}{2}(\bar{R}(a) * C)(\alpha \wedge \alpha) \\
& +\frac{1}{2} u\left(\left[\omega_{k}^{-1}, \omega_{k}^{-1}\right]\right)-\left[u(\alpha), \omega_{k}^{-1}\right]_{k-2} \equiv 0 .
\end{aligned}
$$

" $\equiv 0$ " in this last equality clearly reduces to " $=0 "$. Thus we get

$$
\begin{aligned}
& -C(X \wedge Y)+\left(\bar{R}(a)^{*} C\right)(X \wedge Y)+u\left(\left[X^{-1}, Y^{-1}\right]\right) \\
& -\left[u(X), Y^{-1}\right]+\left[u(Y), X^{-1}\right]=0
\end{aligned}
$$

for any $X, Y \in \mathbb{D}_{k-2}^{-1}$. It follows from (9.15) and (9.16) that $-C$ $+\bar{R}(a)^{*} C-\partial u=0$. We have thereby proved Lemma 9.9.

9.5. In what follows, we shall consider a fixed complementary subspace $\mathfrak{S}_{\sharp}^{(k-1)}$ of $\partial \mathfrak{s}^{k}$ in $\mathfrak{5}^{(k-1)}$. Moreover we assume that the Lie group $G^{0}$ is connected.

Let $P_{\#}^{k}$ denote the subset of $F^{k}$ consisting of all the elements $z$ such that $C(z) \in \mathfrak{C}_{\sharp}^{(k-1)}$. We show that $P_{\sharp}^{k}$ is a $G_{\sharp}^{k}$-subbundle of $F^{k}\left(P^{k-1}\right.$, $\left.H^{k}\right)$. First, we have $\pi\left(P_{\sharp}^{k}\right)=P^{k-1}$. Indeed, let $z \in F^{k}$. Then there is a $u \in \mathfrak{g}^{k}$ such that $C(z)+\partial u \in \mathbb{S}_{\sharp}^{(k-1)}$. If we put $a=t^{k}(u)$, then we have $C(z a)=C(z)+\partial u \in \mathbb{C}_{\sharp}^{(k-1)}$ (Lemma 9.9), which means $z a \in P_{\#}^{k}$. Therefore we must have $\pi\left(P_{\sharp}^{k}\right)=P^{k-1}$. Now, let $z \in P_{\sharp}^{k}$ and $a \in H^{k}$. Let $u \in \mathfrak{g}^{k}$ be such that $a \equiv t^{k}(u)\left(\bmod N^{k}\right)$. By Lemma 9.9, then we see that $z a \in P_{\sharp}^{k}$ if and only if $\partial u=0$, i.e., $u \in \mathrm{g}^{k}$. Therefore $z a \in P_{\#}^{k}$ if and only if $a \in G_{\#}^{k}$. We have thereby proved our assertion. By Lemma 9.8 , we find that the $G_{\#}^{k}$-structure $\left(P_{\#}^{k}, \omega^{(k)}\right)$ on $P^{k-1}$, thus obtained, is of type $\mathrm{m}$. Let $\alpha$ be the projection of $P_{\#}^{k}$ onto $P^{k-1}$, which is just

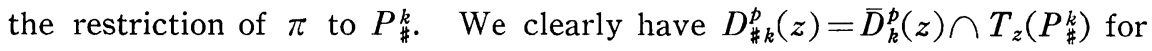
any $z \in P \sharp$ and $p<0$. Therefore, from (9.2) follows that $D_{\sharp k}^{p}=\alpha^{*} D_{k-1}^{p}$ 
$(p<0)$ and ()$_{k}^{p} \mid D_{\# k}^{p-k}=\alpha^{*} \theta_{k-1}^{p}(p<k-1)$.

We have thus shown that to every pseudo- $G^{k-1}$-structure $\left(P^{k-1}\right.$, $\left.D^{(k-1)}, \theta^{(k-1)}\right)$ of type $\| \prime$ on a manifold $M^{k-2}$, there is associated, in a

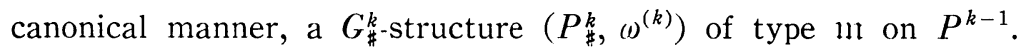

9.6. We must show that the assignment $\left(P^{k-1}, D^{(k-1)}, \theta^{(k-1)}\right) \rightarrow$ $\left(P_{\#}^{k}, \omega^{(k)}\right)$ is compatible with the respective isomorphisms.

Let $\left(P^{k-1}, D^{(k-1)}, \theta^{(k-1)}\right)$ (resp. $\left.\left(P^{\prime k-1}, D^{(k-1)}, \theta^{\prime(k-1)}\right)\right)$ be a pseudo$G^{k-1}$-structure on a manifold $M^{k-2}\left(\right.$ resp. $\left.M^{k-2}\right)$ and let $\left(P_{\#}^{k}, \omega^{(k)}\right)$ (resp.

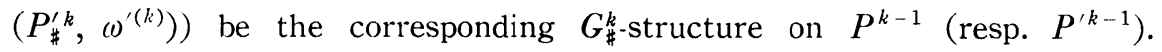
Furthermore, let $\left(F^{k}, \omega^{(k)}\right)$ (resp. $\left(F^{\prime k}, \omega^{\prime(k)}\right)$ ) be the corresponding $H^{k}$. structure on $P^{k-1}$ (resp. $\left.P^{k-1}\right)$. We shall write as $A^{\prime}$ the quantity in $\left(P^{\prime k-1}, D^{\prime(k-1)}, \theta^{\prime(k-1)}\right)$ or $\left(P_{\#}^{\prime k}, \omega^{\prime(k)}\right)$ or $\left(F^{\prime k}, \omega^{\prime(k)}\right)$ which corresponds to a quantity $A$ in $\left(P^{k-1}, D^{(k-1)}, \theta^{(k-1)}\right)$ or $\left(P_{\#}^{k}, \omega^{(k)}\right)$ or $\left(F^{k}, \omega^{(k)}\right)$.

Let $\varphi$ be an isomorphism of $\left(P^{k-1}, D^{(k-1)}, \theta^{(k-1)}\right)$ onto $\left(P^{\prime k-1}\right.$, $\left.D^{\prime(k-1)}, \theta^{\prime(k-1)}\right)$. From the definition of $\left(F^{k},()^{(k)}\right)$, we see that $\varphi$ yields an isomorphism $\bar{\varphi}$ of $\left(F^{k},\left(\omega^{(k)}\right)\right.$ onto $\left(F^{\prime k}, \omega^{\prime(k)}\right)$. We clearly have $C^{\prime}(\bar{\varphi}(z))=C(z)$ for any $z \in F^{k}$. Therefore $\bar{\varphi}$ yields an isomorphism $\varphi_{\sharp}$ of $\left(P_{\#}^{k}, \omega^{(k)}\right)$ onto $\left(P_{\#}^{\prime k}, \omega^{\prime(k)}\right)$. Conversely, let $\varphi_{\#}$ be an isomorphism of $\left(P_{\#}^{k}, \omega^{(k)}\right)$ onto $\left(P_{\#}^{\prime k}, \omega^{\prime(k)}\right)$. Let $\varphi$ be the diffeomorphism of $P^{k-1}$ onto $P^{\prime k-1}$ induced by $\varphi_{\sharp}$. Since $\varphi \circ \alpha=\alpha^{\prime} \circ \varphi_{\#}$ and $\varphi_{\sharp}^{*} \omega^{\prime(k)}=\omega^{(k)}$, we have $\varphi^{*} D_{k-1}^{\prime p}=D_{k-1}^{p}(p<0)$ and $\varphi^{*} \theta_{k-1}^{p \prime}=\theta_{k-1}^{p}(p \leqq k-2)$. Take any $z \in P_{\#}^{k}$ and set $z^{\prime}=\varphi_{\#}(z), x=\alpha(z)$ and $x^{\prime}=\alpha^{\prime}\left(z^{\prime}\right)=\varphi(x)$. Then we have $r^{\prime}\left(X^{k-1}\right)_{x^{\prime}}$ $=z^{\prime} X^{k-1}=\varphi_{*}\left(z \cdot X^{k-1}\right)=\varphi_{*} r\left(X^{k-1}\right)_{x}$ for any $X^{k-1} \in \mathfrak{g}^{k-1}$. Since the group $G^{k-1}$ is connected, it follows that $\varphi(x a)=\varphi(x) a$ for any $x$ $\in P^{k-1}$ and $a \in G^{k-1}$. We have thereby proved $\varphi$ to be an isomophism of $\left(P^{k-1}, D^{(k-1)}, \theta^{(k-1)}\right)$ onto $\left(P^{\prime k-1}, D^{\prime(k-1)}, \theta^{(k-1)}\right)$.

We have thus completed proof of Th. 8.2.

\section{Proof of Lemma 8.2.}

9.7. Hereafter we shall use the notations in Lemma 8.2. Let us

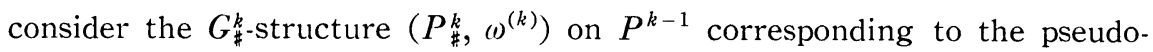
$G^{k-1}$-structure $\left(P^{k-1}, D^{(k-1)}, \theta^{(k-1)}\right)$ (Th. 8.2.). Then the infinitesimal transformation $\sigma^{k-1}(a)$ of $\left(P^{k-1}, D^{(k-1)}, \theta^{(k-1)}\right)$ induces an infinitesimal 
transformation $\sigma_{\#}^{k}(a)$ of $\left(P_{\#}^{k}, \omega^{(k)}\right)$ (Th. 8.2.). Now consider the following proposition $\left(S_{\sharp}^{k}\right)(k \geqq 0)$ : There is a point $z^{k} \in P_{\sharp}^{k}$ such that

$$
\omega_{k}^{r}\left(\sigma_{\#}^{k}\left(a^{p}\right)_{z^{k}}\right)=\delta_{r p} a^{p}
$$

for any $a^{p} \in \mathrm{g}^{p}(p$ arbitrary) and any $r<k$.

Lemma 8.2. follows immediately from the following

Lemma 9.10. (1) $\left(S_{\sharp}^{0}\right)$ is true.

(2) If $\left(S_{\sharp}^{k}\right)$ is true, so is $\left(S^{k}\right)(k \geqq 0)$.

(3) If $\left(S^{k-1}\right)$ is true, so is $\left(S_{\sharp}^{k}\right)(k>0)$.

Before proving Lemma 9.10, we first prove the following

Lemma 9.11. Suppose that $\left(S_{\#)}^{k}\right.$ is true $(k \geqq 0)$. Then we have:

(1) $\sigma_{\#}^{k}\left(a^{k}\right)_{z^{k}}=r_{\#}\left(a^{k}\right)_{z^{k}}$

for any $a^{k} \in \mathrm{g}^{k}$, where, in general, $r_{\sharp}(a)$ denotes the vertical vector field of the principal fiber bundle $P_{\#}^{k}\left(P^{k-1}, G_{\sharp}^{k}\right)$ induced by $a \in \mathrm{g}_{\sharp}^{k}=\mathrm{g}_{\#}^{k}+\mathfrak{n}^{k}$.

(2) $\sigma_{\sharp}^{k}\left(a^{p}\right)_{z^{k}} \equiv 0\left(\bmod r_{\sharp}\left(\mathfrak{n}^{k}\right)_{z^{k}}\right)$

for any $a^{p} \in \mathfrak{g}^{p}(p>k)$.

Proof. Let $a^{p} \in \mathfrak{g}^{p}(p \geqq k)$. By $\left(S_{\sharp}^{k}\right)$, we see that $\sigma_{\sharp}^{k}\left(a^{p}\right)_{z^{k}}$ is a vertical vector in $P_{\#}^{k}\left(P^{k-1}, G_{\sharp}^{k}\right)$. Therefore we can find an $\bar{a}^{p} \in \mathrm{g}_{\sharp}^{k}$ such that $\sigma_{\#}^{k}\left(a^{p}\right)_{z^{k}}=r_{\#}\left(\bar{a}^{p}\right)_{z^{k}}$. We have $\left.r_{\#}\left(\bar{a}^{p}\right)\right\lrcorner d \omega^{(k)}+\bar{a}^{p} \cdot \omega^{(k)}=0$ and $\left.\left.L_{\sigma_{\sharp}^{k}\left(a^{p}\right)} \omega^{(k)}=\sigma_{\sharp}^{k}\left(a^{p}\right)\right\lrcorner d \omega^{(k)}+d\left(\sigma_{\sharp}^{k}\left(a^{p}\right)\right\lrcorner \omega^{(k)}\right)=0$. Let $b^{q} \in \mathrm{g}^{q}(q<0)$. Then we have $L_{\sigma_{\sharp}^{k}\left(b^{q}\right)} \omega^{(k)}=0$ and $\left[\sigma_{\#}^{k}\left(a^{p}\right), \sigma_{\#}^{k}\left(b^{q}\right)\right]=-\sigma_{\#}^{k}\left(\left[a^{p}, b^{q}\right]\right)$. By using these equalities, we get

$$
-\omega^{(k)}\left(\sigma_{\#}^{k}\left(\left[a^{p}, b^{q}\right]\right)_{z^{k}}\right)+\bar{a}^{p} \cdot b^{q}=0 .
$$

From (9.18) and $\left(S_{\sharp}^{k}\right)$, it follows that $\left[a^{k}, b^{q}\right]=\bar{a}^{k} \cdot b^{q}$, whence $a^{k}=\bar{a}^{k}$. Moreover it follows that $\bar{a}^{p} \cdot b^{-1}=0$ for any $p>k$, whence $\bar{a}^{p} \in \mathfrak{n}^{k}$. We have thereby proved Lemma 9.11 .

Proof of Lemma 9.10. Let $\alpha^{k}\left(\right.$ resp. $\left.\beta^{k}\right)$ be the projection of $P_{\#}^{k}$ 
onto $P^{k-1}$ (resp. $\left.P^{k}\right)$.

(1) Let $\xi$ be the Maurer-Cartan form of the Lie group $M(\mathrm{~m})$ and let $g$ be the cross-section of $P_{\#}^{0}\left(M(\mathrm{~m}), G_{\sharp}^{0}\right)$ which was observed in 4.4. Put $z^{0}=g(e)$ and let $a^{p} \in \mathrm{g}^{p}$. Then we have $\omega_{0}^{r}\left(\sigma_{\#}^{0}\left(a^{p}\right)_{z^{0}}\right)=\xi^{r}\left(\sigma^{-1}\left(a^{p}\right)_{e}\right)$ $=f_{\sigma^{-1}\left(a^{p}\right)}^{r}(e)=\delta_{r p} a^{p}$. Therefore we have proposition $\left(S_{\sharp}^{0}\right)$ for the point $z^{0}$.

(2) Put $e^{k}=\beta^{k}\left(z^{k}\right)$ and take a cross-section $h$ of $P_{\sharp}^{k}\left(P^{k}, N^{k}\right)$ such that $h\left(e^{k}\right)=z^{k}$. Then $\xi^{(k)}=h^{*} \omega^{(k)}$ is compatible with $\theta^{(k)}$. Let $a^{p} \in \mathfrak{g}^{p}$. Then we have $\xi_{k}^{r}\left(\sigma^{k}\left(a^{p}\right)_{e^{k}}\right)=\omega_{k}^{r}\left(\sigma_{\#}^{k}\left(a^{p}\right)_{z^{k}}\right) \delta_{r p} a^{p}$. Moreover by $\left(S_{\sharp}^{k}\right)$ and Lemma 9. 11, it is clear that $\sigma^{k}\left(a^{k}\right)_{e^{k}}=r\left(a^{k}\right)_{e^{k}}$ and $\sigma^{k}\left(a^{p}\right)_{e^{k}}=0$ for any $p>k$. Thus we have proposition $\left(S^{k}\right)$ for the pair $\left(e, \xi^{(k)}\right)$.

(3) Let $\left(F^{k}, \omega^{(k)}\right)$ be the $H^{k}$-structure on $P^{k-1}$ corresponding to the pseudo- $G^{k-1}$-structure $\left(P^{k-1}, D^{(k-1)}, \theta^{(k-1)}\right)$ and let us use the notations in 9.3-9.6. Let $\bar{\sigma}(a)$ denote the infinitesimal transformation of $\left(F^{k}, \omega^{(k)}\right)$ induced by $\sigma^{k-1}(a)$. First of all, we shall show that there is a point $z^{k} \in F^{k}$ such that

$$
\omega_{k}^{r}\left(\bar{\sigma}\left(a^{p}\right)_{z^{k}}\right)=\delta_{r p} a^{p}
$$

for any $a^{p} \in \mathfrak{g}^{p}\left(r<k, p\right.$ arbitrary). Indeed if we put $\xi^{p}=\pi^{*} \xi_{k-1}^{p}$, then we have $\omega_{k}^{p} \equiv \bar{\xi}^{p}\left\{\bmod \xi^{r}(r \leqq p-k)\right\}$ for any $p \leqq k-2$. Take a point $y \in F^{k}$ such that $\pi(y)=e^{k-1}$. Then it follows from this fact and $\left(S^{k-1}\right)$ that there is $a \tau \in H^{k}$ such that $\omega^{(k)}\left(\bar{\sigma}(a)_{y}\right)=\tau a$ for any $a \in \mathrm{m}^{k-2}$. Put $z^{k}=y \tau$. Since $R_{\tau *} \bar{\sigma}(a)_{y}=\bar{\sigma}(a)_{z^{k}}$ and $R_{\tau}^{*} \omega^{(k)}=\tau^{-1} \omega^{(k)}$, we get

$$
\omega^{(k)}\left(\bar{\sigma}(a)_{z^{k}}\right)=a
$$

Let $a^{k-1} \in \mathrm{g}^{k-1}$. Then by $\left(S^{k-1}\right)$ and Lemmas 9.3 and 9.5 , we have

$$
\omega^{(k)}\left(\bar{\sigma}\left(a^{k-1}\right)_{z^{k}}\right)=\omega^{(k)}\left(\hat{r}\left(a^{k-1}\right)_{z^{k}}\right)=a^{k-1} .
$$

Let $a^{p} \in \mathrm{g}^{p}(p \geqq k)$. Then by $\left(S^{k-1}\right)$, we have

$$
\omega^{(k)}\left(\bar{\sigma}\left(a^{p}\right)_{z^{k}}\right)=0
$$


(9.3) now follows from (9.20), (9.21) and (9.22), proving our assertion. Let us prove $z^{k} \in P_{\#}^{k}$. For this purpose, it suffices to show that $\left(\Omega_{k}^{p}\right)_{z^{k}}$ $\equiv 0\left\{\bmod \left(\omega_{k}^{r}\right)_{z^{k}}(r \leqq p-k)\right\}$ for any $p \leqq k-2$. But, this is easy from (9.19) and the following facts:

$$
[\bar{\sigma}(a), \bar{\sigma}(a)]=-\bar{\sigma}([a, b]) ; L_{\bar{\sigma}(a)}\left(\omega^{(k)}=0 ; \hat{r}(u)\right\lrcorner \Omega_{k}^{p} \equiv 0
$$

$\left\{\bmod \omega_{k}^{r}(r \leqq p-k)\right\}$ for any $u \in \mathfrak{h}^{k}$. Finally it is clear from (9.19) that we have proposition $\left(S_{\#}^{k}\right)$ for the point $z^{k}$.

We have thus completed the proof of Lemma 8.2.

\section{§10. Applications to the geometry of real submanifolds of complex manifolds}

10.1. In this section, we shall always assume the differentiability of class $C^{\omega}$.

Let $f$ be an imbedding of a real manifold $M$ to a complex manifold $\tilde{M}$. Let $x$ be any point of $M$. Then the tangent space $T_{f(x)}(\tilde{M})$ is a complex vector space and the image $f_{*} T_{x}(M)$ of the tangent space $T_{x}(M)$ by the differential $f_{*}$ of $f$ is a real subspace of $T_{f(x)}(\tilde{M})$. We denote by $D(f, x)$ the maximum complex subspace of $T_{f(x)}(\tilde{M})$ contained in $f_{*} T_{x}(M)$, i.e.,

$$
D(f, x)=f_{*} T_{x}(M) \cap \sqrt{-1} f_{*} T_{x}(M),
$$

and, throughout this section, assume that $\operatorname{dim}_{C} D(f, x)$ is constant.

The notations being as above, we now define a subspace $D(x)$ of $T_{x}(M)$ by $f_{*} D(x)=D(f, x)$ and a complex structure $I_{x}$ on the vector space $D(x)$ by $f_{*} I_{x} X=\sqrt{-1} f_{*} X$ for all $X \in D(x)$. Then we see that the assignment $x \rightarrow D(x)$ gives a differential system $D$ on $M$ and the assignment $x \rightarrow I_{x}$ gives a cross-section $I$ of the vector bundle Hom $(D, D)$. Denotes by $D^{0,1}$ the subbundle of the complexified tangent bundle $T^{c}(M)$ of $M$ which consists of all the vectors $X+\sqrt{-1} I X$ $(X \in D)$. Then we have $f_{*} D^{0,1}(x)=T^{0,1}(f(x)) \cap f_{*} T_{x}^{c}(M)$, where $T^{0,1}$ denotes the bundle of tangent vectors of type $(0,1)$ associated with the 
almost complex structure on $\tilde{M}$. Since the almost complex structure is integrable, we have $\left[\underline{T}^{0,1}, \underline{T}^{0,1}\right] \subset \underline{T}^{0,1}$. It follows that $\left[\underline{D}^{0,1}, \underline{D}^{0,1}\right]$ $C \underline{D}^{0,1}$, which is equivalent to the following two statements: For any two local cross-sections $X$ and $Y$ of $D$,

(10.1) 1) $[I X, I Y]-[X, Y]$ is a local cross-section of $D$,

2) $[I X, I Y]-[X, Y]=I([I X, Y]+[X, I Y])$.

Definition 10.1. (1) Let $D$ be a differential system on a manifold $M$ and let $I$ be a cross-section of $\operatorname{Hom}(D, D)$. Then the pair $(D, I)$ is called an almost pseudo-complex structure on $M$ if $I_{x}$ is a complex structure on $D(x)$ and if $I$ satisfies $(10.1), 1)$. Moreover an almost pseudo-complex structure $(D, I)$ is called integrable or a pseudo-complex structure if it also satisfies (10.1), 2).

(2) Let $(D, I)$ (resp. $\left(D^{\prime}, I^{\prime}\right)$ ) be an almost pseudo-complex structure on a manifold $M\left(\right.$ resp. $\left.M^{\prime}\right)$. Then an isomorphism $\varphi$ of $(M, D)$ onto $\left(M^{\prime}, D^{\prime}\right)$ is called an isomorphism of $(M, D, I)$ onto $\left(M^{\prime}, D^{\prime}, I^{\prime}\right)$ if $\varphi_{*} I X=I^{\prime} \varphi_{*} X(X \in D)$.

By the above argument, we know that with every imbedding $f$ of a real manifold $M$ to a complex manifold $\tilde{M}$ there is associated a pseudo-complex structure $(D, I)$ on $M$ in a natural manner. Note that a pseudo-complex structure (resp. an almost pseudo-complex structure) is essentially the same thing as an integrable $H$-structure $(F, \omega)$ (resp. a $H$-structure $(F, \omega)$ satisfying condition $(C))$ in $[13], \S 8$.

10.2. Let $(D, I)$ be a pseudo-complex structure on a manifold $M$ and let us consider the subbundle $D^{0,1}$ of $T^{c}(M)$. Let $M^{c}$ be a (sufficiently small) complexification of the real analytic manifold $M$ (cf. [15]), which is a complex manifold of complex dimension $\mathfrak{m}=\operatorname{dim} M$ such that $M$ is imbedded in $M^{c}$ as a real part, i.e., $T_{x}\left(M^{c}\right)=T_{x}(M)$ $+\sqrt{-1} T_{x}(M)=T_{x}^{c}(M)$ at each $x \in M$. Then the subbundle $D^{0,1}$ of $T^{c}(M)$ is extended to a holomorphic subbundle $E$ of $T\left(M^{c}\right)$ just as in the case of an almost complex manifold. Since $\left[\underline{D}^{0,1}, \underline{D}^{0,1}\right] \subset \underline{D}^{0,1}$, we find that $E$ is completely integrable, considered as a holomorphic differential 
system on $M^{c}$.

Proposition 10.1. (cf. [13], Remark 2). Let $f$ be an imbedding of a real manifold $M$ to a complex manifold $\tilde{M}$ (such that $\operatorname{dim}_{C} D(f, x)$ $=$ constant), and let $(D, I)$ be the corresponding pseudo-complex structure on $M$. Then there is a complex submanifold $\tilde{M}_{0}$ of $\tilde{M}$ such that $f(M)$ $\subset \tilde{M}_{0}$ and such that

$$
\operatorname{dim} M=\operatorname{dim}{ }_{C} \tilde{M}_{0}+\operatorname{dim}{ }_{C} D(f, x) \quad(x \in M) .
$$

Moreover, $\tilde{M}_{0}$ is uniquely determined as a germ.

Proof. The notations being as above, $f$ is extended to a holomorphic mapping $f^{c}$ of $M^{c}$ to $\tilde{M}$. At each $x \in M$, the differential $f_{* x}^{c}$ of $f^{c}$ at $x$ is a complex extention of the differential $f_{*_{x}}$ of $f$ at $x$. Since $f_{*} I Y=\sqrt{-1} f_{*} Y(Y \in D(x))$, it follows that the kernel of $f_{*_{x}}^{c}$ is equal to $D^{0,1}(x)=E(x)$. Since $E$ is a holomorphic extention of $D^{0,1}$, we see that the kernel of $f_{*_{y}}^{c}$ is equal to $E(y)$ at each $y \in M^{c}$. Therefore the image $\tilde{M}_{0}$ of $M^{c}$ by $f^{c}$ is a complex submanifold of $\tilde{M}$ whose dimension is equal to $\operatorname{dim} M-\operatorname{dim}{ }_{c} E(x)=\operatorname{dim} M-\operatorname{dim}{ }_{C} D(f, x)$. We clearly have $f(M) \subset \tilde{M}_{0}$. Let $\tilde{M}_{0}^{\prime}$ be a second submanifold of $\tilde{M}$ satisfying the condition in Prop. 10.1. Then $(D, I)$ coincides with the pseudocomplex structure corresponding to the imbedding $f: M \rightarrow \tilde{M}_{0}^{\prime}$. Therefore it is clear from the above argument that $\tilde{M}_{0} \subset \tilde{M}_{0}^{\prime}$ as a germ. Since $\operatorname{dim}{ }_{C} \tilde{M}_{0}=\operatorname{dim}{ }_{c} \tilde{M}_{0}^{\prime}$, we must have $\tilde{M}_{0}=\tilde{M}_{0}^{\prime}$ as a germ.

Proposition 10.2. (cf. [13], Remark 5). Let $(D, I)$ be any pseudo-complex structure on a manifold $M$. Then there are a complex manifold $\tilde{M}$ and an imbedding $f$ of $M$ to $\tilde{M}$ such that the imbedding $f$ satisfies

$$
\operatorname{dim} M=\operatorname{dim}{ }_{c} \tilde{M}+\operatorname{dim}{ }_{c} D(f, x) \quad(x \in M)
$$

and such that the given $(D, I)$ is just the pseudo-complex structure on $M$ corresponding to the imbedding $f$.

Proof. After a suitable arrangement of $M^{c}$, we have only to 
define $\tilde{M}$ as the manifold $M^{c} / E$ of all the leaves of the completely integrable differential system $E$ and $f$ as the natural mapping from $M$ to $M^{c} / E$.

Proposition 10.3. (cf. [13], Lemma 8.2). Let $f\left(\right.$ resp. $\left.f^{\prime}\right)$ be an imbedding of a real manifold $M$ (resp. $M^{\prime}$ ) to a complex manifold $\tilde{M}\left(\right.$ resp. $\left.\tilde{M}^{\prime}\right)$ satisfying (10.2) and let $(D, I)\left(\right.$ resp. $\left.\left(D^{\prime}, I^{\prime}\right)\right)$ be the corresopnding pseudo-complex structure on $M\left(\right.$ resp. $\left.M^{\prime}\right)$. Let $\varphi$ be a homeomorphism of $M$ onto $M^{\prime}$. Then $\varphi$ is an isomorphism of $(M, D, I)$ onto $\left(M^{\prime}, D^{\prime}, I^{\prime}\right)$ if and only if there is a holomorphic homeomorphism $\tilde{\varphi}$ of a neighborhood of $f(M)$ onto a neighborhood of $f^{\prime}\left(M^{\prime}\right)$ such that $\tilde{\varphi} \circ f=f^{\prime} \circ \varphi$. Moreover, $\tilde{\varphi}$ is uniquely determined by $\varphi$ as a germ.

Proof. $\varphi$ is extended to a holomorphic homeomorphism $\varphi^{c}$ of $M^{c}$ onto $M^{\prime c}$ assuming that $M^{c}$ and $M^{\prime c}$ are suitably chosen. Then $\varphi$ is an isomorphism of $(M, D, I)$ onto $\left(M^{\prime}, D^{\prime}, I^{\prime}\right)$ if and only if $\varphi^{c}$ is an isomorphism of $\left(M^{c}, E\right)$ onto $\left(M^{\prime c}, E^{\prime}\right)$. This last statement is equivalent to the existence of a holomorphic homeomorphism $\tilde{\varphi}$ of a neighborhood of $f(M)$ onto a neighborhood of $f^{\prime}\left(M^{\prime}\right)$ such that $\tilde{\varphi} \circ f^{c}=f^{\prime c} \circ \varphi^{c}$. Prop. 10.3 is clear from these arguments.

Analogously we have

Proposition 10.4. (cf. [11], Prop. 1). Let $f$ be an imbedding of a real manifold $M$ to a complex manifold $\tilde{M}$ satisfying (10.2) and let $(D, I)$ be the corresponding pseudo-complex structure on $M$. Let $X$ be a vector field on $M$. Then $X$ is an infinitesimal automorphism of $(M, D, I)$ if and only if there is a holomorphic vector field $\tilde{X}$ defined on a neighborhood of $f(M)$ such that $X$ and $\tilde{X}$ are $f$-related, i.e., $f_{*} X_{x}=\tilde{X}_{f(x)}$ at each $x \in M$.

10.3. Let $(D, I)$ be an almost pseudo-complex structure on a manifold $M$ and assume that the differential system $D$ is regular. Let us consider the fundamental graded algebra $\mathfrak{m}(x)=\sum_{p<0} \mathfrak{g}^{p}(x)$ at any point $x \in M$. Then we have $\mathfrak{g}^{-1}(x)=D(x)$ and, by $\left.(10.1), 1\right)$,

$$
\left[I_{x} X, I_{x} Y\right]=[X, Y] \quad(\text { in } \mathfrak{m}(x))
$$


for all $X, Y \in \mathfrak{g}^{-1}(x)$. For each $\alpha \in \mathfrak{g}^{-2}(x)^{*}$, define a bilinear form $H^{\alpha}$ on $\mathfrak{g}^{-1}(x)$ by $H^{\alpha}(X, Y)=\alpha\left(\left[I_{x} X, Y\right]\right)$ for all $X, Y \in \mathfrak{g}^{-1}(x)$. Then $H^{\alpha}$ is a hermitian form on $\mathfrak{g}^{-1}(x)$ and is called the Levi-form of $(D, I)$ at $x$ with respect to $\alpha$.

Let $\mathfrak{m}=\sum_{p<0} \mathfrak{g}^{p}$ be a fundamental graded algebra over $\boldsymbol{R}$ and let $I$ be a complex structure on $\mathfrak{g}^{-1}$. Then the pair $(n, I)$ is called a pseudocomplex fundamental graded algebra if it satisfies the equality

$$
[I X, I Y]=[X, Y]
$$

for all $X, Y \in \mathfrak{g}^{-1}$. Let $(n, I)$ (resp. $\left(\mathrm{nt}^{\prime}, I^{\prime}\right)$ ) be a pseudo-complex fundamental graded algebra. An isomorphism $\varphi$ of $m$ onto $\mathrm{m}^{\prime}$ (as graded algebras) is called an isomorphism of ( $\mathrm{n}, I)$ onto $\left(\mathrm{n}^{\prime}, I^{\prime}\right)$ if $I^{\prime} \varphi X=\varphi I X$ for all $X \in \mathfrak{q}^{-1}$.

Let $(\mathrm{n}, I)$ be a pseudo-complex fundamental graded algebra. Then we say that an almost pseudo-complex structure $(D, I)$ on a manifold $M$ is of type $(n, I)$ if $D$ is regular and if $\left(n(x), I_{x}\right)$ is isomorphic with $(n, I)$ at every $x \in M$. We denote by $G^{0}(n, I)$ the group of all the automorphisms of $(n, I)$, being a subgroup of $G^{0}(n)$, and put $G_{\sharp}^{0}(\mathrm{~m}, I)=G^{0}(\mathrm{~m}, I) N^{0}$. Furthermore, we denote by $\mathfrak{g}^{0}(\mathfrak{m}, I)$ the Lie algebra of $G^{0}(\mathfrak{m}, I)$, and by $\mathfrak{g}(\mathfrak{m}, I)$ the prolongation of $\left(\mathfrak{m}, \mathfrak{g}^{0}(\mathfrak{m}, I)\right)$.

Proposition 10.5. (nt, I) being as above, let $M$ be a manifold of dimension $m=\operatorname{dim} \| \mathrm{t}$. Then an almost pseudo-complex structure $(D, I)$ on $M$ of type $(\mathrm{nt}, I)$ is characterized as a $G_{\#}^{0}(\mathrm{~m}, I)$-structure of type int on $M$.

This is easy from the arguments in $\S 4$.

By Th. 8.4 and Prop. 10.5, we have

Proposition 10.6. (cf. [3]; [11]; [12]; [13], Prop. 8.1). (m, I) being as above, let $(D, I)$ be an almost pseudo-complex structure of type $(\mathrm{n}, I)$ on a connected manifold $M$ of dimension $m=\operatorname{dim} n \mathrm{n}$. If (n, $\left.g^{0}(\mathrm{n}, I)\right)$ is of finite type, then the Lie algebra of all the infinitesimal automorphisms of $(M, D, I)$ is finite dimensional and of dimension 
$\leqq \operatorname{dim} \mathfrak{g}(\sharp, I)$.

In $\S 11$, we shall see that $\left(n, \mathfrak{g}^{0}(n, I)\right)$ is of finite type if and only if $n$ is non-degenerate, i.e., the condition " $X \in \mathfrak{g}^{-1},\left[X, g^{-1}\right]=0$ " implies $X=0$.

10.4. Let (nI, I) be a pseudo-complex fundamental graded algebra. Let us observe the standard differential system $D$ of type $m$ on the Lie group $M(111)$. Then the complex structure $I$ on $a^{-1}$ defines a crosssection of $\operatorname{Hom}(D, D)$, denoted by the same letter $I$, which is invariant under the left translations of $M(I I)$. It is clear that the pair $(D, I)$ is a pseudo-complex structure of type $(n 1, I)$ on $M(n 1)$, which is called the standard pseudo-complex structure of type (11I,I). It can be shown that the standard $G_{\#}^{0}(n t, I)$-structure of type $\|$ on $M(n)$ just corresponds to the standard pseudo-complex structure $(D, I)$. Therefore the Lie algebra sheaf $\mathscr{L}$ of all local infinitesimal automorphisms of $(D, I)$ coincides with the standard Lie algebra sheaf of type $\left(n, \mathrm{~g}^{0}(n, I)\right)$.

By Th. 6.2, we have

Proposition 10.7. The formal algebra of $\mathscr{L}$ may be identified with the formal algebra associated with the prolongation $\mathrm{g}(\mathrm{nI}, I)$ of $\left(\mathrm{nt}, \mathfrak{g}^{0}(\mathrm{nt}, I)\right)$.

\section{\$11. Some results on certain graded modules}

11.1. In this section, $K$ will denote any field of characteristic zero.

Let $\Pi=\sum_{r<0} \mathfrak{g}^{r}$ be a fundamental graded (Lie) algebra over $K$. A vector space $E$ over $K$ is called a right $" 1$-module if the Lie algebra $\|$ is represented on the vector space $E$ in the right, i.e., if there is given a bilinear mapping $E \times \| \ni(a, x) \rightarrow a x \in E$ as follows:

$$
(a x) y-(a y) x=a[x, y]
$$

for all $a \in E$ and $x, y \in$ II. Analogously we have the notion of a left m-module.

Let $E$ be a right $n$-module. A direct sum $E=\sum_{p} E^{p}$, the indices $p$ 
taking any integers, is called a right graded $m$-module if it satisfies the following conditions:

1) $\operatorname{dim} E^{p}<\infty$;

2) $E^{p} \mathrm{~g}^{r} \subset E^{p+r}$.

Furthermore a direct sum $E=\sum_{p=k}^{l} E^{p}$ is called a right graded m-module if the direct sum $E=\sum_{p} E^{p}$, where $E^{p}=0(p<k$ or $p>l)$, gives a right graded $\mathrm{m}$-module.

We say that a (right) graded $m$-module $E=\sum_{p} E^{p}$ satisfies condition $(C)$ if it satisfies the following conditions:

1) $E^{p}=\{0\}$ for all $p<-\mu$, if $\mathrm{nt}$ is of $\mu$-th kind;

2) For any $p \geqq 0$, the condition " $a \in E^{p}, a \mathfrak{g}^{-1}=\{0\}$ " implies $a=0$.

Let $E=\sum_{p} E^{p}$ be a graded $\mathrm{ml}$-module satisfying condition $(C)$. Then $E$ is called of infinite type (resp. of finite type) if $E^{p} \neq\{0\}$ for all $p \geqq 0$ (resp. if $E^{p_{0}}=\{0\}$ for some $p_{0} \geqq 0$ ). If $E^{p_{0}}=\{0\}$ for some $p_{0}$ $\geq 0$, it is clear that $E^{p}=\{0\}$ for all $p>p_{0}$.

Examples. (1). Let $\mathfrak{n t}$ and $F^{-1}$ be two finite dimensional vector spaces. Let $F^{0}$ be a subspace of $\operatorname{Hom}\left(n, F^{-1}\right)$ and let $F^{(p)}(p>0)$ be the $p$-th prolongation of the subspace $F^{0} C \operatorname{Hom}\left(n, F^{-1}\right)$. Then we have natural bilinear mappings $F^{(p)} \times \Uparrow \ni(a, x) \rightarrow a x \in F^{(p-1)}$ for all $p \geqq 0$, where $F^{(-1)}=F^{-1}$ and $F^{(0)}=F^{0}$, and the following equalities: $(a x) y$ $=(a y) x$ for all $a \in F^{(p)}(p>0)$ and $x, y \in \mathfrak{l}$. Suppose now that we are given subspaces $F^{p} C F^{(p)}(p>0)$ as follows: $F^{p} \mathfrak{l} C F^{p-1}$. If we consider the vector space $\|$ as an abelian Lie algebra and further as a fundamental graded algebra of first kind, then we see that the direct sum $F=\sum_{p=-1}^{\infty} F^{p}$ is endowed with a structure of graded $\mathfrak{n}$-module and that it satisfies condition $(C)$. Such a graded module has been investigated in [5] and [9]. Conversely, let $F=\sum_{p=-1}^{\infty} F^{p}$ be any graded n-module satisfying condition $(C)$, where $\mathrm{n}$ is a fundamental graded algebra of first 
kind. Then $F^{0}$ may be identified with a subspace of Hom (nt, $F^{-1}$ ) and $F^{p}(p>0)$ with a subspace of the $p$-th prolongation $F^{(p)}$ of $F^{0}$.

(2) Let $\mathfrak{g}=\sum_{p} \mathfrak{g}^{p}$ be a graded (Lie) algebra over $K$ satisfying the following conditions:

1) $\mathrm{n}=\sum_{r<0} \mathrm{~g}^{r}$ is a fundamental graded algebra;

2) For each $p \geq 0$, the condition " $a \in \mathfrak{g}^{p},\left[a, \mathfrak{g}^{-1}\right]=0$ " implies $a=0$.

Then $\mathfrak{g}=\sum_{p} \mathfrak{g}^{p}$ becomes a graded $m$-module with respect to the bilinear mapping $\mathfrak{g} \times \mathfrak{n} \ni(a, x) \rightarrow a x=[a, x] \in \mathfrak{g}$ and satisfies condition $(C)$.

Remark 1. Let $E_{0}=\sum_{p=-\mu}^{0} E^{p}$ be a graded m-module such that the condition " $\left.a \in E^{0}, a\right]^{-1}=0$ " implies $a=0$. In the same way as in $\S 5$, then it can be shown that the graded 111-module $E_{0}$ is prolonged to a graded nt-module $E=\sum_{p=-\mu}^{\infty} E^{p}$ which satisfies condition $(C)$ and which is maximal in an appropriate sense.

11.2. Let $E=\sum_{p} E^{p}$ be a graded nt-module satisfying condition $(C)$. Put $E^{-1}=H^{-1}(E)$ and denote by $H^{p}(E)(p \geqq 0)$ the subspace of $E^{p}$ consisting of all $a \in E^{p}$ such that $a \Im^{r}=\{0\}$ for all $r<-1$. Then we have $H^{p}(E) \mathfrak{g}^{-1} \subset H^{p-1}(E)$ for all $p \geqq 0$ and $(a x) y=(a y) x$ for all $a$ $\in H^{p}(E)(p>0)$ and $x, y \in \mathfrak{a}^{-1}$. Therefore the direct sum $H(E)=\sum_{p=-1}^{\infty} H^{p}(E)$ is endowed with a structure of graded $\mathfrak{g}^{-1}$-module, where $\mathfrak{g}^{-1} \stackrel{p=-1}{\text { should be }}$ considered as a fundamental graded algebra of first kind. It clearly satisfies condition $(C)$. If $n$ t is of first kind, then $H(E)$ and $E$ coincide.

The main purpose of the present section is to prove the following

Theorem 11.1. Let $\mathrm{m}=\sum_{r<0} \mathrm{~g}^{r}$ be a fundamental graded algebra over $K$ and let $E=\sum_{p} E^{p}$ be a graded 11 -module satisfying condition $(C)$. If the graded m-module $E$ is of infinite type, so is the graded $\mathfrak{g}^{-1}$-module $H(E)$.

Corollary 1. Let $\mathfrak{g}=\sum_{p} \mathfrak{g}^{p}$ be a graded algebra over $K$ satisfying $(11.1), 1)$ and 2$)$. Let $\mathfrak{h}^{p}(p \geqq 0)$ be the subspace of $\mathfrak{g}^{p}$ consisting of all 
$a \in \mathfrak{g}^{p}$ such that $\left[a, \mathfrak{g}^{r}\right]=\{0\}$ for all $r<-1$, i.e., $\mathfrak{h}^{p}=H^{p}(\mathfrak{g})$. If the Lie algebra $\mathrm{g}$ is infinite dimonsional, then we have $\mathfrak{h}^{p} \neq\{0\}$ for all $p \geq 0$.

This is clear from 'Th. 11.1 .

Corollary 2. Let $\Pi=\sum_{r<0} \mathrm{~g}^{r}$ be a fundamental graded algebra over $K$ and let $\mathrm{g}^{0}$ be a subalgebra of the Lic algebra $\mathrm{g}^{0}(\mathrm{nt})$ of all derivations of the graded algebra 11 . Let $\mathfrak{h}^{0}$ be the ideal of $\mathfrak{g}^{0}$ consisting of all $a \in \mathfrak{g}^{0}$ such that $\left[a, \mathfrak{g}^{r}\right]=0$ for all $r<-1$. We identify $\mathfrak{h}^{0}$ with a subspace of $\operatorname{Hom}\left(\mathfrak{g}^{-1}, \mathfrak{g}^{-1}\right)$ (by identifying $\left.a \in \mathfrak{h}\right)^{0}$ with the linear mapping $\left.\mathfrak{g}^{-1} \ni x \rightarrow[a, x] \in \mathfrak{g}^{-1}\right)$. Then the pair $\left(n, \mathfrak{g}^{0}\right)$ is of infinite type if and only if the subspace $\mathfrak{h}^{0} C \operatorname{Hom}\left(\mathfrak{g}^{-1}, \mathfrak{g}^{-1}\right)$ is of infinite type.

Indeed, let $\mathfrak{g}=\sum_{p} \mathfrak{g}^{p}$ be the prolongation of $\left(\mathfrak{m}, \mathfrak{g}^{0}\right)$. It is easy to see that $\mathfrak{h}^{p}=H^{p}(\mathfrak{g})(p>0)$ coincides with the $p$-th prolongation $\mathfrak{h}^{(p)}$ of $\mathfrak{h}^{0}=H^{0}(\mathfrak{g})$. Therefore Cor. 2 is immediate from Cor. 1.

We say that a fundamental graded algebra $n t=\sum_{r<0} \mathfrak{g}^{r}$ is nondegenerate if the condition " $x \in \mathfrak{g}^{-1},\left[x, \mathfrak{g}^{-1}\right]=0$ " implies $x=0$.

Corollary 3. Let $\mathrm{m}=\sum_{r<0} \mathrm{~g}^{r}$ be a fundamental graded algebra over $\boldsymbol{R}$ and suppose that there is given a complex structure $I$ on the vector space $\mathfrak{g}^{-1}$ such that $[I x, I y]=[x, y]$ for all $x, y \in \mathfrak{g}^{-1}$. Let $\mathfrak{g}^{0}$ be the subalgebra of $\mathfrak{g}^{0}(\mathrm{nII})$ consisting of all $a \in \mathrm{g}^{0}(\mathrm{nI})$ such that aIx $=$ Iax for all $x \in \mathfrak{g}^{-1}$, i.e., $\mathrm{g}^{0}$ is equal to the subalgebra $\mathrm{g}^{0}(\mathrm{n}, I)$ of $\mathfrak{g}^{0}(\mathrm{nI})$ defined in $\S 10$. Then the pair $\left(\mathrm{nI}, \mathrm{g}^{0}\right)$ is of finite type if and only if the fundamental graded algebra $\mathrm{nt}$ is non-degenerate.

Indeed, it is easy to see that $\left(n, \mathfrak{g}^{0}\right)$ is of infinite type if $m$ is degenerate. Suppose that $m$ is non-degenerate and let $g=\sum_{p} g^{p}$ be the prolongation of $\left(u, \mathfrak{g}^{0}\right)$. The notations being as above, let us show that $\mathfrak{h}^{1}=\mathfrak{h}^{(1)}$ vanishes. In fact, put $\langle x, y\rangle=[I x, y]$ for all $x, y \in \mathfrak{g}^{-1}$. Then we have $\langle x, y\rangle=\langle y, x\rangle$ and the condition " $x \in \mathfrak{g}^{-1},\left\langle x, \mathfrak{g}^{-1}\right\rangle$ $=\{0\}$ " implies $x=0$. Thus $<,>$ is, so to speak, a $\mathfrak{g}^{-1}$-valued inner product on the vector space $\mathfrak{g}^{-1}$. We have $\langle a x, y\rangle+\langle x, a y\rangle=0$ for all $a \in \mathfrak{h}^{0}$ and $(b x) y=(b y) x$ for all $b \in \mathfrak{h}^{1}$. It follows that 
$\langle(b x) y, z>=0 \text { for all } b \in \mathfrak{h})^{1}, x, y, z \in \mathfrak{g}^{-1}$ (cf. the proof of the fact that the first prolongation $\mathfrak{v}(n, \boldsymbol{R})^{(1)}$ of the orthogonal algebra $\mathfrak{v}(n, \boldsymbol{R})$ vanishes), whence $b=0$. We have thereby proved $\mathfrak{h}^{1}=0$. Therefore by Cor. 2 , we know that $\left(n, \mathfrak{g}^{0}\right)$ is of finite type.

Remark 2. Cor. 2 is fundamental in the classification of infinite primitive pseudo-groups (See Morimoto and Tanaka [6]). It has been announced in the preprint of the present paper; Our initial proof of Cor. 2 made use of Lemma 2 in Guillemin, Quillen and Sternberg [4] which was proved by an analytical method. Cor. 3 is important in the geometry of real submanifolds of complex manifolds (See §10).

11.3. The proof of Th. 11.1 is preceded by several lemmas. In this paragraph, " will denote any finite dimensional vector space over $K$, identified with a fundamental graded algebra of first kind.

Let $F=\sum_{p=-1}^{\infty} F^{p}$ be a graded $\mathfrak{u}$-module satisfying condition $(C)$. Given a base $u_{1}, \ldots, u_{n}$ of $\mathfrak{u}$, denote by $F_{i}^{p}(p \geqq 0,0 \leqq i \leqq n)$ the subspace of $F^{p}$ consisting of all $a \in F^{p}$ such that $a e_{1}=\cdots=a e_{i}=0$. Then we have $F_{0}^{p}=F^{p}, F_{n}^{p}=\{0\}$ and $F_{i}^{p} \leadsto \subset F_{i}^{p-1} ; F_{i+1}^{p}$ can be characterized as the kernel of the linear mapping $F_{i}^{p} \ni a \rightarrow a u_{i+1} \in F_{i}^{p-1}$.

We say that a graded $\mathfrak{n}$-module $F=\sum_{p=-1}^{\infty} F^{p}$ satisfying condition $(C)$ is in involution if it satisfies the following conditions:

1) $F^{p}$ is equal to the $p$-th prolongation $F^{(p)}$ of $F^{0}$ for every $p>0$;

2) It admits a regular base for $F$, i.e., there is a base $u_{1}, \ldots, u_{n}$ of " such that the linear mappings $F_{i}^{p} \ni a \rightarrow a u_{i+1} \in F_{i}^{p-1}(0 \leqq i<n, p$ $>0)$ are surjective.

Let $F=\sum_{p=-1}^{\infty} F^{p}$ be any graded $\Uparrow$-module satisfying condition $(C)$. Let $q$ be any integer $>0$. Then the graded $\llbracket$-module $F(q)=F / \sum_{p<q-1} F^{p}$ $=\sum_{p=-1}^{\infty} F^{q+p}$ clearly satisfies condition $(C)$. It is known that $F(q)$ becomes in involution for any sufficiently large $q([9])$.

Lemma 11.1. Let $F_{(\alpha)}=\sum_{p=-1}^{\infty} F_{(\alpha)}^{p}(0 \leqq \alpha<\mu)$ be $\mu$ graded $\mathfrak{u}$-modules satisfying condition $(C)$. Let $V$ and $W$ be two finite dimensional vector 
spaces and let $\varphi$ be a bilinear mapping of $V \times W$ to $n$ such that the vector space $\mathfrak{i t}$ is generated by the vectors of the form $\varphi(v, w)(v \in V$, $w \in W)$. If $F_{(\alpha)}^{p} \|=F_{(\alpha)}^{p-1}$ for all $\alpha$ and $p>0$, then there are $a v \in V$ and $a w \in W$ such that the linear mappings $F_{(\alpha)}^{p} \ni a \rightarrow a \varphi(v, w) \in F_{(\alpha)}^{p-1}$ are surjective for all $\alpha$ and $p>0$.

In general, let $F=\sum_{p=-1}^{\infty} F^{p}$ be any graded $\mathfrak{n}$-module which satisfies condition $(C)$ and which is such that $F^{p_{1}}=F^{p-1}$ for all $p>0$. It is known that there are proper subspaces $P_{1}, \ldots, P_{e}$ of $\|$ having the following property ([5], Appendix, Lemma 1): If $x \in \|$ and if $x \notin P_{1}$ $\cup \cdots \cup P_{e}$ then $x$ is regular for $F$, i.e., the linear mappings $F^{p} \ni a \rightarrow a x$ $\in F^{p-1}$ are surjective for all $p>0$. Therefore we can find a finite set $\Delta$ of non-zero linear forms on $\|$ such that $x \in \mathfrak{n}$ is regular for every $F_{(\alpha)}$ provided $\omega(x)=0$ for all $\omega \in \Delta$. Lemma 11.1 is easy from this fact.

Lemma 11.2. Let $F_{(\alpha)}, V, W$ and $\varphi$ be as in Lemma 11.1. If $F_{(\alpha)}$ is in involution for every $\alpha$, then there are $v_{i} \in V$ and $w_{i} \in W$ $(1 \leqq i \leqq n)$ such that the $n$ vectors $u_{i}=\varphi\left(v_{i}, w_{i}\right)$ form a regular base for every $F_{(\alpha)}$.

This lemma follows from Lemma 11.1. and the theory of modules in involution ( $[5]$ or $[9]$ ).

11.4 Let $m=\sum_{r=-\mu}^{-1} \mathrm{~g}^{r}$ be a fundamental graded algebra of $\mu$-th kind over $K(\mu>1)$ and let $U$ be the universal enveloping algebra of it. Let $M$ be a left m-module, which is also a left $U$-module. Assume that $M$ is graded: $M=\sum_{p \geqq 0} M^{p}$, the indices $p$ taking values $\geqq 0 ; \operatorname{dim} M^{p}<\infty$; $\mathrm{g}^{r} M^{p} C M^{p-r}$. For each $x \in U$, denote by $x^{(p)}$ the linear mapping $M^{p}$ $\ni a \rightarrow a x \in M$. When $x \in \mathrm{g}^{r}, x^{(p)}$ maps $M^{p}$ into $M^{p-r}$.

Lemma 11.3. Let $v \in \mathfrak{g}^{-1}$ and $w \in \mathfrak{g}^{-\mu+1}$ and put $u=[v, w]\left(\in \mathfrak{g}^{-\mu}\right)$. If $u^{(p)}: M^{p}: \rightarrow M^{p+\mu}$ are injective for all $p \geqq 0$, then, for every $p \geqq 0$, there is a $t \in K$ such that $\left(v^{\mu-1}+t w\right)^{(p)}: M^{p} \rightarrow M^{p+\mu-1}$ is injective.

Proof. It is sufficient to prove that there is a $t \in K$ such that $\left(v^{\mu-1}+t w\right)^{(0)}$ is injective. Suppose that $\left(v^{\mu-1}+t w\right)^{(0)}$ are not injective 
On differential systems, graded Lie algebras and pseudo-groups 79 for all $t \in K$. As is easily observed, then there is a polynomial mapping $f$ of $K$ to $M^{0}$ such that $\left(v^{\mu-1}+t w\right) f(t)=0$ for all $t \in K$ and such that $f(0) \neq 0$. Let us express $f(t)$ as $\sum_{i=0}^{k} t^{i} a_{i}$, where $a_{i} \in M^{0}$. Then we have $a_{0}=f(0) \neq 0$ and

$$
v^{\mu-1} a_{i}+w a_{i-1}=0 \quad(0 \leqq i \leqq k+1)
$$

where we put $a_{-1}=a_{k-1}=0$. Since $v w-w v=u$ and $v u=u v$, we have

$$
v^{i} w=w v^{i}+i u v^{i-1}
$$

By (11.2) and (11.3), we have

$$
v^{\mu(i+1)-1} a_{i}+(w v+\mu i u) v^{\mu i-1} a_{i-1}=0 .
$$

Since $a_{-1}=0$, it follows that

$$
v^{\mu(i+1)-1} a_{i}=0 \quad(0 \leqq i \leqq k)
$$

We have $a_{k+1}=0$ and assert that $a_{k}=0$. Indeed, we have $v^{\mu(k+1)-1} a_{k}$ $=w a_{k}=0$ by (11.2) and (11.4). $i$ being an integer with $0 \leqq i<\mu(k+1)$ -1 , suppose that $v^{i+1} a_{k}=0$. By (11.3), then we have

$$
v^{i+1} w a_{k}=w v^{i+1} a_{k}+(i+1) u v^{i} a_{k},
$$

whence $u v^{i} a_{k}=u^{(i)} v^{i} a_{k}=0$. Since $u^{(i)}$ is injective, this gives $v^{i} a_{k}$ $=0$. Thus we get $v^{i} a_{k}=0(0 \leqq i \leqq \mu(k+1)-1)$, proving our assertion. Therefore by induction, we get $a_{i}=0(0 \leqq i \leqq k)$, which is a contradiction.

Lemma 11.4. Let $v, w$ and $u$ be as in Lemma 11.3. If $u^{(p)}$ are isomorphisms for all $p \geqq 0$, then we have $M=\{0\}$.

Proof. By Lemma 11.3, we easily find $\operatorname{dim} M^{p}=$ constant. We have $v w-w v=u, v u=u v$ and $w u=u w$, whence 


$$
\begin{aligned}
& v^{(p+\mu-1)} w^{(p)}-w^{(p+1)} v^{(p)}=u^{(p)}, \\
& v^{(p+\mu)} u^{(p)}=u^{(p+1)} v^{(p)}, \\
& \left.w^{(p+\mu)} u^{(p)}=u^{(p+\mu-1}\right) w^{(p)} .
\end{aligned}
$$

Let us identify $M^{p+\mu}$ with $M^{p}$ by the isomorphism $u^{(p)}$ for every $p$ $(0 \leqq p<\mu)$ and denote by $1_{p}$ the identity transformation of $M^{p}$. Then we have $v^{(p+\mu)}=v^{(p)}(0 \leqq p \leqq \ell-1)$ and $w^{(\mu)}=w^{(0)}$ and hence

$$
\begin{aligned}
& v^{(\mu-1)} w^{(0)}-w^{(1)} v^{(0)}=1_{0}, \\
& v^{(p-1)} w^{(p)}-w^{(p+1)} v^{(p)}=1_{p} \quad(0<p<\mu-1), \\
& v^{(\mu-2)} w^{(\mu-1)}-w^{(0)} v^{(\mu-1)}=1_{\mu-1} .
\end{aligned}
$$

Since $\operatorname{dim} M^{p}=$ constant, we may further identify $M^{p}$ with $M^{0}$ (by an arbitrary isomorphism) for every $p(0<p<\ell)$. By (11.5), then we have

$$
\left(v^{(\mu-1)} w^{(0)}-w^{(0)} v^{(\mu-1)}\right)+\sum_{p=0}^{\mu-2}\left(v^{(p)} w^{(p+1)}-w^{(p+1)} v^{(p)}\right)=1_{0}
$$

If follows that $\iota \operatorname{dim} M^{0}=\varkappa \operatorname{Tr} 1_{0}=0$, whence $M^{0}=\{0\}$. We have thus proved $M^{p}=\{0\}$ and hence $M=\{0\}$.

11.5. Proof of Theorem 11.1. Let $m=\sum_{r=-\mu}^{-1} \mathfrak{g}^{r}$ be a fundamental graded algebra of $\mu$-th kind over $K$ and let $E=\sum_{p=-\mu}^{\infty} E^{p}$ be a graded $\Pi$ module satisfying condition $(C)$. We shall prove $T$ h. 11.1 by induction on the integer $\mu$. If $\mu=1$, then $H(E)=E$ and Th. 11.1 is the case. Therefore we may assume that $\mu>1 . \mathfrak{g}^{-\mu}$ being a graded ideal of $n$, the factor space $m_{*}=n / \mathrm{g}^{-\mu}=\sum_{r=-\mu+1}^{-1} \mathrm{~g}^{r}$ is endowed with a structure of graded algebra, which is a fundamental graded algebra of $(\mu-1)$-th kind. Put $E^{p}=E_{0}^{p}(p<0)$ and denote by $E_{0}^{p}(p \geqq 0)$ the subspace of $E^{p}$ consisting of all $a \in E^{p}$ such that $a \mathfrak{g}^{-\mu}=\{0\}$. Then we have $E_{0}^{p} \mathrm{~g}^{-\mu}$ $=\{0\}$ and $E_{0}^{p} \mathfrak{g}^{r} \subset E_{0}^{p+r}$ for all $p$ and all $r<0$. Therefore we see that the direct $\operatorname{sum} E_{*}=\sum_{p=-\mu+1}^{\infty} E_{0}^{p}$ is endowed with a structure of graded $\mathfrak{m}_{*}$ - 
module, which satisfies condition $(C)$. We have $H(E)=H\left(E_{*}\right)$. Consequently to prove Th. 11.1, it is sufficient to prove that the graded m-module $E$ is of finite type, assuming the same for the graded $\mathfrak{m}_{*}$ module $E_{*}$.

Assume that $E_{*}$ is of finite type and hence that $E_{0}^{p_{0}}=\{0\}$ for some $q_{0} \geqq 0$. Then we have $E_{0}^{q}=\{0\}$ for all $q \geqq q_{0}$, i.e., the condition " $a \in E^{q}, a \mathfrak{g}^{-\mu}=\{0\}$ " implies $a=0$. Let $\alpha$ and $q$ be such that $0 \leqq \alpha<\mu$ and $q \geqq q_{0}$. We have $E^{\alpha+q+\mu p} \mathfrak{g}^{-\mu} C E^{\alpha+q+\mu(p-1)}$ and hence the direct $\operatorname{sum} \sum_{p} E^{\alpha+q+\mu \phi}$ is a graded $\mathfrak{g}^{-\mu}$-module, where the abelian subalgebra $\mathfrak{g}^{-\mu}$ of $\mathrm{nt}$ should be considered as a fundamental graded algebra of first kind. It follows that the direct sum $E(\alpha, q)=\sum_{p=-1}^{\infty} E^{\alpha+q+\mu p}$ is also a graded $\mathfrak{g}^{-\mu}$-module (cf. the graded $\mathfrak{n}$-module $\stackrel{p=-1}{F(q)}$ in 11.3). Since $E_{0}^{\alpha+q+\mu p}=\{0\}$ for all $p \geqq 0$, the graded $\mathfrak{g}^{-\mu}$-module $E(\alpha, q)$ satisfies condition $(C)$.

Therefore for a sufficiently large $q$, the $\mu$ graded $\mathfrak{g}^{-\mu}$-modules $E(\alpha, q)(0 \leqq \alpha<\mu)$ become in involution simultaneously. Fix such a $q$ from now on. Since $\mathfrak{g}^{-\mu}=\left[\mathfrak{g}^{-1}, \mathfrak{g}^{-\mu+1}\right]$, it follows from Lemma 11.2 that there are $v_{i} \in \mathfrak{q}^{-1}$ and $w_{i} \in \mathfrak{q}^{-\mu+1}(1 \leqq i \leqq n)$ such that the $n$ vectors $u_{i}=\left[v_{i}, w_{i}\right]$ form a regular base for every $E(\alpha, q)$. Let $E_{i}^{k}(0 \leqq i \leqq n)$, $k \geqq q$ ) denote the subspace of $E^{k}$ consisting of all $a \in E^{k}$ such that $a u_{1}=\cdots=a u_{i}=0$. Then we have $E_{i}^{k} \mathrm{~g}^{r} \subset E_{i}^{k+r}(0 \leqq i \leqq n, r<0, k+r$ $\geqq q)$. Since the $\mu$ graded $\mathfrak{q}^{-\mu}$-modules $E(\alpha, q)$ are in involution, the linear mappings $E_{i}^{k} \ni a \rightarrow a u_{i+1} \in E_{i}^{k-\mu}$ are surjective for all $k \geqq q+\mu$. Let $j$ be the smallest $i(0 \leqq i<n)$ such that $E_{i+1}^{q}=\{0\}$. Since $E_{j+1}^{k} \mathrm{~g}^{-1}$ $C E_{j+1}^{k-1}$, then we find $E_{j+1}^{k}=\{0\}$ for all $k \geqq q$. It follows that the linear mappings $E_{j}^{k} \ni a \rightarrow a u_{j+1} \in E_{j}^{k-\mu}$ are isomorphisms for all $k \geqq q+\mu$.

Let us now prove $E_{j}^{k}=\{0\}$ for all $k \geqq q$. Denote by $M^{p}(p \geqq 0)$ the dual space of $E_{j}^{p+q}$ and put $M=\sum_{p \geqq 0} M^{p}$ (direct sum). For any $m \in M^{p}$ and $x \in \mathrm{g}^{r}$, define an element $x m$ of $M^{p-r}$ by $(x m)(a)=m(a x)$ for all $a \in E_{j}^{q+p-r}$. Then the bilinear mappings $\mathfrak{g}^{r} \times M^{p} \ni(x, m) \rightarrow x m$ $\in M^{p-r}(p \geqq 0, r<0)$ give rise to a structure of left graded m-module on $M$. The notations being as in 11.4 , the linear mappings $u_{j+1}^{(p)}$ are 
isomorphisms for all $p \geqq 0$. Since $u_{j+1}=\left[v_{j+1}, w_{j+1}\right]$, Lemma 11.4 can be applied to the graded $11 \mathrm{t}$-module $M$ and we have $M^{p}=\{0\}$ for all $p \geqq 0$. Hence $E_{j}^{k}=\{0\}$ for all $k \geqq q$.

Therefore we have $j=0$ on account of the way of choosing the integer $j$. Hence $E^{k}=E_{0}^{k}=\{0\}$ for all $k \geqq q$. We have thereby proved that the graded $\mathrm{m}$-module $E$ is of finite type and have completed the proof of Th. 11.1.

\section{Bibliography}

[1] E. Cartan, Les systèmes pfaff á cinq variables et les équations aux derivées partielles du second ordre, Ann. Ec. Normale, 27 (1910), 109-192.

[2] E. Cartan, Sur l'équivalence absolue de certains systèmes d'équations différentielles et sur certaines familles de courbes, Bull. Soc. Math. France, 42 (1914), 12-48.

[3] E. Cartan, Sur la géométrie pseudo-conforme des hypersurfaces de l'espace de deux variables complexes, I, Annali di Math., 11 (1932), 17-90.

[4] V. Guillemin, D. Quillen and S. Sternberg, The classification of the complex primitive infinite pseudo-groups, Proc. Nat. Acad. Sciences, 55 (1966), 687690.

[5] V. Guillemin and S. Sternberg, An algebraic model of transitive differential geometry, Bull. Amer. Math. Soc., 70 (1964), 16-47.

[6] T. Morimoto and N. Tanaka, The classification of real primitive infinite Lie algebras, J. Math. Kyoto Univ. 10-2 (to appear).

[7] T. Ochiai, On the automorphism group of a $G$-structure, J. Math. Soc. Japan 18 (1966), 189-193.

[8] E. A. Ruh, On the automorphism group of a $G$-structure, Comment. Math. Helv. 39 (1964), 189-264.

[9] I. M. Singer and S. Sternberg, The infinite group of Lie and Cartan, J. Analyse Math., 15 (1965), 1-114.

[10] S. Sternberg, Lectures on differential geometry, New Jersey, Prentice-Hall, Inc. (1964).

[11] N. Tanaka, On the pseudo-conformal geometry of hypersurfaces of the space of $n$ complex variables, J. Math. Soc. Japan, 14 (1962), 397-429.

[12] N. Tanaka, Graded Lie algebras and geometric structures, Proc. U. S.Japan Seminar in Diff. Geometry (1965), 147-150.

[13] N. Tanaka, On generalized graded Lie algebras and geometric structures I, J. Math. Soc. Japan, 19 (1967), 215-254.

[14] N. Tanaka, On infinitesimal automorphisms of Siegel domains, J. Math. Soc. Japan, 22 (1970) 180-212.

[15] H. Whitney and F. Bruhat, Quelques propriétés fondamentales des ensembles analytiques-réels, Comment. Math. Helv. 33 (1959), 132-160.

Kyoto University 